\title{
Quantum Mechanics in Space-Time: the Feynman Path Amplitude Description of Physical Optics, de Broglie Matter Waves and Quark and Neutrino Flavour Oscillations
}

\author{
J.H.Field \\ Département de Physique Nucléaire et Corpusculaire Université de Genève . 24, quai \\ Ernest-Ansermet CH-1211 Genève 4.
}

\begin{abstract}
Feynman's laws of quantum dynamics are concisely stated, discussed in comparison with other formulations of quantum mechanics and applied to selected problems in the physical optics of photons and massive particles as well as flavour oscillations. The classical wave theory of light is derived from these laws for the case in which temporal variation of path amplitudes may be neglected, whereas specific experiments, sensitive to the temporal properties of path amplitudes, are suggested. The reflection coefficient of light from the surface of a transparent medium is found to be markedly different to that predicted by the classical Fresnel formula. Except for neutrino oscillations, good agreement is otherwise found with previous calculations of spatially dependent quantum interference effects.
\end{abstract}

PACS 03.65.Bz, 14.60.Pq, 14.60.Lm, 13.20.Cz

Keywords ; Quantum Mechanics, Physical Optics. 


\section{Introduction}

In 1983, Richard Feynman was invited to give the first series of Alix J. Mautner Memorial Lectures at the University of California, Los Angeles. These lectures were subsequently published as the book: 'QED The Strange Theory of Light and Matter' [1]. They are a masterpiece of popular science. The experiment Feynman chose to describe in detail was reflection from, and transmission of light through, glass sheets of various thicknesses, at near-normal incidence. Closely analogous experiments were first performed and analysed by Isaac Newton $[2]^{1}$. After a simplified analysis, the complete quantum mechanical calculation for the experiments is presented in Chapter 3 of [1]. This is done in terms of 'arrows' (vectors in the complex plane) that represent quantum amplitudes in one-to-one correspondence with paths where a photon is scattered from each atom of the sheet of glass. No equations are used. To the present writer's best knowledge, this is the only place, in Feynman's writings or elsewhere, where this calculation may be found. When Feynman had earlier discussed the reflection and transmission of light in 'The Feynman Lectures in Physics' [3] he had presented the standard text-book analysis in terms of surface boundary conditions on the electric and magnetic fields of electromagnetic waves, leading to the Fresnel formulae for the reflection and transmission coefficients.

The principal aim of the present paper is to work out, in full mathematical detail, the QED predictions for some of the experiments discussed in [1]. It is found, perhaps surprisingly, that the reflection coefficient of light at a vacuum/glass interface, calculated by the path amplitude method described in Chapter 3 of [1], is in marked disagreement with the Fresnel formula!

The essential ideas whose applications were developed in [1] were already concisely stated [4] in 'The Feynman Lectures in Physics'. In describing the path amplitude interpretation of quantum mechanics in these lectures however [5], Feynman prefered to discuss non-relativistic electrons rather than photons, in correspondence with his published work on the Path Integral approach $[6,7]$ that was restricted to non-relativistic quantum mechanics. With the exception of the short passage cited above with the revelatory title 'How it Works' light was always described in [3] in terms of electromagnetic waves, not photons.

Feynman stated that the path integral formulation of quantum mechanics is not only the most fundamental, but also the easiest to understand. Why then has its impact, both on the teaching of physics ${ }^{2}$ and on the presentation of quantum mechanical subjects in the research literature been so limited? This is true even for subjects where the path amplitude approach would seem to be the most natural one, such as physical optics and quark flavour and neutrino oscillations in particle physics. The answer to this question

\footnotetext{
${ }^{1}$ These experiments actually studied interference effects of light crossing a thin air film trapped between spherical and plane glass surfaces -the well-known 'Newton's Rings'. The quantum mechanical analysis of these experiments is essentially identical to that of those discussed by Feynman in [1]. The jacket of the first edition of this book showed a coloured photograph of thin film interference effects produced by a thin oil slick.

${ }^{2}$ An important exception to this is the work of E.F.Taylor and collaborators [8] who have stressed the insight into classical mechanics and Hamilton's Principle provided by Feynman's formulation of quantum mechanics.
} 
is perhaps related to the fact that the situations in which the path amplitude formalism is both transparent and powerful -quantum predictions for correlated observations in space-time, which may be termed 'quantum dynamics', is far removed from the problem that was confronted and solved by the founders of quantum mechanics: Bohr, Heisenberg, Schrödinger and Pauli. This problem was to describe the structure of atoms and to calculate the rates of radiative transitions between different atomic energy levels, a subject which could be termed 'quantum statics' since the detailed space-time structure of fundamental processes does not play any important role ${ }^{3}$. Indeed the latter subject is not discussed at all in the vast majority of text-books on quantum mechanics ${ }^{4}$. If future research physicists are never systematically taught Feynman's formulation of quantum mechanics it is not surprising that they remain forever ignorant of it. When some brief discussion of, say, particle propagation in space-time, is included in standard text-books, concepts such as spatial 'wave packets', relevant only to certain classes of physical problems, are often introduced, in a prefectly general way, leading, in some cases, to erroneous conclusions. This point will be considered in more detail in the following section.

Feyman's formulation of quantum mechanics is more fundamental and powerful than the earlier formulations of Heisenberg and Schrödinger because, as Feynman showed, starting from the path integral formula Schrödinger's equation and therefore all of 'quantum statics' is readily derived. However knowledge of Schrödinger's equation alone is, by far, not sufficient to obtain correct predictions for space-time correlated events. This is demonstated by the different predictions for 'neutrino oscillations' to be found in the published literature and text books $[11,12,13]$.

Before the work presented in the present paper, the present writer is aware of only two papers in which the Feynman path integral formalism is formally applied to optical problems. The first [14] concerns the refraction of light and the second [15] the optics of beams of non-relativistic particles. This being said, it remains true that in the description of almost any experiment in quantum optics involving multiple photon paths, path amplitudes are tacitly employed, usually called instead 'photon fields', 'wave functions' or 'state vectors'. The most detailed explicit application of the Feynman path integral method known to the present writer is in atomic physics: the 'Photodetachement Microscope' [16].

In all of the optical experiments to be described in the following, as is the case in [1], the initial state is assumed to be a single excited atom which subsequently decays spontaneously producing a single photon. This photon interacts with the experimental apparatus and later the same, or a different, single photon is detected. Also as in [1], for simplicity, only experiments where the effects of atomic or photon polarisation are inessential, and may be neglected, are considered.

\footnotetext{
${ }^{3}$ Bohr made the following remark on this subject: 'For example the experiments regarding the excitation of spectra by electronic impacts and by radiation are adequately accounted for on the assumption of discrete stationary states and individual transition processes. This is primarily due to the circumstance that in these questions no closer description of the space-time behaviour of the processes is required.' $[9]$.

${ }^{4}$ This is not the case for [10], an introductory text book largely based on Feynman's space-time approach. As in Section 2 below, Feynman's rules for constructing probability amplitudes are explicitly stated in this book.
} 
The structure of the paper is as follows: In the following section Feynman's principles of quantum dynamics and their interpretation are reviewed. Thus the basic formulae used to perform the calculations presented in the subsequent sections are given. In Section 3 the invariant propagator of a free on-shell particle is derived from the relativistic Feynman path integral, and the temporal propagator of a particle at rest from the time-dependent Schrödinger Equation. In Section 4 the classical wave theory of light in which only spatial 'waves' are considered is derived from the Feynman path integral. The calculation, from first principles, of the refractive index of a uniform transparent medium is performed in Section 5. A new phenomenon 'refraction annulment' which may occur when the excited atom decays promptly after production, is predicted. The laws of reflection, refraction and linear propagation of light are derived in Section 6. A quantitative estimation is made of the spread of the photon paths around the classical trajectory of ray optics corresponding to an extremum of the phase of the path amplitude. In Section 7 the calculation of the reflection coefficient of light at normal incidence at the interface of two transparent media, as qualitatively sketched in [1] is presented. In Section 8 time-dependent variation of the fringe visiblity in a Michelson Interferometer, using as a light source spontaneously decaying excited atoms produced at a known time, is calculated. This type of prediction, testing essential temporal features of the path amplitude formalism, cannot be obtained using the classical wave theory of light. In Section 9 the path amplitude method is extended to the description of de Broglie matter waves and heavy quark and neutrino flavour oscillations. In each case a 'two probability amplitude' experiment is analysed in detail. These are: Young double slit experiments using photons or electrons, neutral kaon flavour oscillations in the processes $\pi^{-} p \rightarrow \Lambda\left(\mathrm{K}_{S}, \mathrm{~K}_{L}\right), \mathrm{K}_{S}, \mathrm{~K}_{L} \rightarrow \pi^{ \pm} e^{\mp} \nu$ and neutrino oscillations in the processes: $\pi^{+} \rightarrow \mu^{+} \nu, \nu n \rightarrow e^{-} p$. A summary and outlook are given in Section 10.

As this paper is a long one, the reader is recommended to look first at the concluding section for an overview, returning later to any earlier sections that contain material of particular interest.

\section{Feynman's Formulation of the Laws of Quantum Dynamics and Their Interpretation}

In Chapter 3 of Volume 3 of the 'The Feynman Lectures in Physics' [3] Feynman set down four general principles for the quantum description of space-time processes. These are rules concerning the meaning and the method of construction of the Probability Amplitude that provides the quantum description of any space-time experiment. Any such experiment is defined by a fixed (prepared) initial state $|i\rangle$ and a fixed (measured) final state $|f\rangle$. The probability amplitude, $A_{f i}$, is a complex number that is constructed by summing all Path Amplitudes that have the same initial state $|i\rangle$ and the same final state $|f\rangle$. The path amplitudes, $P A_{f i}$, are, in turn, constructed by multiplying together a time-ordered sequence of Process Amplitudes. Feynman's four principles then define the physical meaning of the probability amplitude and specify how it is constructed:

I The probability, $P_{f i}$, to measure the state, $|f\rangle$, given the prepared state, $|i\rangle$, is:

$$
P_{f i}=\left|A_{f i}\right|^{2}
$$


In general the probability amplitude depends on the space-time evolution of the state $|i\rangle$, which is a function of the spatial geometry and other physical characteristics of the experimental apparatus as well as of those of various process amplitudes where particles may be scattered, destroyed or created. It therefore does not correspond simply to any observable property of either the initial or final state and so should not be confused with the wavefunction of any quantum system.

The path amplitude is constructed according to the law of Sequential Factorisation. If the state $|i\rangle$ evolves in time via a series of unobserved intermediate states: $\left|k_{j}\right\rangle, j=1, n$, the corresponding path amplitude: $\left\langle f\left|k_{n}, \ldots, k_{2}, k_{1}\right| i\right\rangle$ is given by a product of process amplitudes: $\left\langle k_{j+1}\left|T_{j+1}\right| j\right\rangle$, where $T_{j+1}$ is a transition operator that evolves the state $\left|k_{j}\right\rangle$ into $\left|k_{j+1}\right\rangle$. These process amplitudes are either the space-time propagators of particles or the invariant amplitudes of particle decay or scattering ${ }^{5}$ processes that are calculated in momentum space according to the Feynman rules of QED or the Standard Electroweak Model. Since the latter have no space-time dependence they appear only as constant multiplicative factors in the path amplitudes. It is understood that all unobserved particle states in the process amplitudes that are not members of the chain of sequential states $\left|k_{j}\right\rangle, j=1, n$ that define the amplitude, are to be integrated over. Since the corresponding integrals are space-time independent that contribute only a multiplicative constant to $P_{f i}{ }^{6}$.

II Sequential Factorisation:

$$
P A_{f i} \equiv\left\langle f\left|k_{n}, \ldots, k_{2}, k_{1}\right| i\right\rangle=\left\langle f\left|T_{f}\right| k_{n}\right\rangle \ldots\left\langle k_{2}\left|T_{2}\right| k_{1}\right\rangle\left\langle k_{1}\left|T_{1}\right| i\right\rangle
$$

The third principle is Quantum Mechanical Superposition. If several different series of unobserved intermediate states are allowed by the experimental apparatus, the probability amplitude is given by the sum over all such series of intermediate states:

III Quantum Mechanical Superposition:

$$
A_{f i}=\sum_{k_{n}} \ldots \sum_{k_{2}} \sum_{k_{1}}\left\langle f\left|k_{n}, \ldots, k_{2}, k_{1}\right| i\right\rangle
$$

The fourth principle applies if the initial and final states are composite, constructed from the tensor products of sets of states: $\left|i^{(1)}\right\rangle,\left|i^{(2)}\right\rangle \ldots\left|f^{(1)}\right\rangle,\left|f^{(2)}\right\rangle \ldots$ but the path amplitudes linking the pairs $\left|i^{(1)}\right\rangle,\left|f^{(1)}\right\rangle ;\left|i^{(2)}\right\rangle,\left|f^{(2)}\right\rangle ; \ldots$ have no intermediate states in common:

IV Composite Factorisation:

$$
P A_{f^{(1)} f^{(2)} \ldots i^{(1)} i^{(2)} \ldots}=\prod_{j} P A_{f^{(j)} i^{(j)}}
$$

\footnotetext{
${ }^{5}$ The scattering process may be elastic or inelastic.

${ }^{6}$ In the examples discussed in the present paper no account is taken of these integrations. The calculated space-time quantum interference effects are unaffected by this omission
} 
An important special case of this principle occurs when a common prepared initial state gives rise to a number of separately measured final states; i.e. $\left|i^{(1)}\right\rangle,\left|i^{(2)}\right\rangle, \ldots \rightarrow|i\rangle$, in (2.4), but the paths linking $|i\rangle$ to $\left|f^{(1)}\right\rangle,\left|f^{(2)}\right\rangle$, .. are distinct, with no common intermediate states. This results, when quantum experiments containing identical particles are considered, in what is conventionally termed an 'entangled wavefunction'. Examples are the decays of ortho- and para-positronium:

$$
e^{+} e^{-}\left(1^{3} S_{1}\right) \rightarrow \gamma \gamma \gamma, \quad e^{+} e^{-}\left(1^{1} S_{0}\right) \rightarrow \gamma \gamma
$$

In these and similar processes quantum statistics must be respected. In the case of the observation of both photons from the decay of para-positronium, two different path amplitudes are possible:

$$
\begin{aligned}
P A(12)_{f^{(1)} f^{(2)} i}= & \left\langle f^{(1)}\left|T_{D}\right| \gamma_{1}\right\rangle\left\langle\gamma_{1}\left|T\left(P_{1}\right)\right| \gamma_{1}\right\rangle\left\langle\gamma_{1}\left|T_{P}\right| i\right\rangle \\
& \times\left\langle f^{(2)}\left|T_{D}\right| \gamma_{2}\right\rangle\left\langle\gamma_{2}\left|T\left(P_{2}\right)\right| \gamma_{2}\right\rangle\left\langle\gamma_{2}\left|T_{P}\right| i\right\rangle \\
P A(21)_{f^{(1)} f^{(2)} i}= & \left\langle f^{(1)}\left|T_{D}\right| \gamma_{2}\right\rangle\left\langle\gamma_{2}\left|T\left(P_{1}\right)\right| \gamma_{2}\right\rangle\left\langle\gamma_{2}\left|T_{P}\right| i\right\rangle \\
& \times\left\langle f^{(2)}\left|T_{D}\right| \gamma_{1}\right\rangle\left\langle\gamma_{1}\left|T\left(P_{2}\right)\right| \gamma_{1}\right\rangle\left\langle\gamma_{1}\left|T_{P}\right| i\right\rangle
\end{aligned}
$$

In these formulae, $\gamma_{1}$ and $\gamma_{1}$ are the two identical and indistinguishable decay photons, $|i\rangle$ the initial para-positronium state, $\left|f_{1}\right\rangle$ and $\left|f_{2}\right\rangle$ the final states of the photon detection processes, occuring at the ends of the paths, $P_{1}$ and $P_{2}$, through the experimental apparatus. $T_{P}$ and $T_{D}$ are the transition operators of the positronium decay and photon detection processes respectively, while $T\left(P_{1}\right)$ and $T\left(P_{2}\right)$ are photon transition operators for the paths $P_{1}$ and $P_{2}$ respectively. Bose-Einstein statistics requires that the path amplitude, $P A_{f^{(1)} f^{(2)} i}$, for the experiment is symmetric under the exchange $\gamma_{1} \leftrightarrow \gamma_{2}$. Thus

$$
P A_{f^{(1)} f^{(2)} i}=P A(12)_{f^{(1)} f^{(2)} i}+P A(21)_{f^{(1)} f^{(2)} i}
$$

In an experiment where two identical fermions are both detected Fermi-Dirac statisics requires the path amplitude to be anti-symmetric under exchange of the two fermions. The similar path amplitudes $P A(12)_{f^{(1)}} f^{(2)} i$ and $P A(21)_{f^{(1)}} f^{(2)} i$ should then be subtracted rather than added as in (2.7), to yield the path amplitude $P A_{f^{(1)} f^{(2)} i}$ for the experiment.

In practice, experimental set-ups do not usually correspond to the preparation and measurement of a unique intial state and a unique final state, but rather sets of such states: $\left|i_{l}\right\rangle, l=1,2 \ldots(I) ;\left|f_{m}\right\rangle, m=1,2 \ldots(F)$ are prepared or measured, respectively. The appropriate prediction to be compared with experiment is then:

$$
P_{F I}=\sum_{m} \sum_{l}\left|\sum_{k_{n}} \ldots \sum_{k_{2}} \sum_{k_{1}}\left\langle f_{m}\left|k_{n}, \ldots, k_{2}, k_{1}\right| i_{l}\right\rangle\right|^{2}
$$

The incoherent sums over $l$ and $m$ correspond to the density matrices of initial and final states respectively. (2.8) is the basic formula that is used for the calculations presented below. It is a simple iteration of a formula first given by Heisenberg in 1930 [17] which was later adopted by Feynman as the basis for his space-time formulation of quantum mechanics [6].

The principles I-IV above describe the rules for constructing probability amplitudes, but are devoid of any dynamical content. This is provided by a fifth principle, the Feynman Path Integral, which gives the physical prescription to calculate the probability amplitude: 
V The Feynman Path Integral [18]:

$$
A_{f i}=\int_{\text {paths }}\left\langle f\left|\exp \left\{i \frac{S\left[\vec{x}_{p}(t)\right]}{\hbar}\right\}\right| i\right\rangle \prod_{p} \prod_{j=1}^{3} \mathcal{D}\left(x_{p}^{j}(t)\right)
$$

where $^{7}$

$$
S \equiv \int_{t_{i}}^{t_{f}} L\left(\vec{x}_{1}, \dot{\vec{x}}_{1}, \vec{x}_{2}, \dot{\vec{x}}_{2}, \ldots \vec{x}_{p}, \dot{\vec{x}}_{p}, \ldots\right) d t
$$

and

$$
\mathcal{D}(x)=\operatorname{Lim}(\epsilon \rightarrow 0) \iint \ldots \iint \frac{d x_{0}}{A} \frac{d x_{1}}{A} \ldots \frac{d x_{j-1}}{A} d x_{j}
$$

where $x_{0}, x_{1}, \ldots$ denote successive positions along the path each separated by a small fixed time interval, $\epsilon$, and $\mathrm{A}$ is a normalisation constant. The latter will be determined for the case of a free, relativistic, on-shell particle in the following Section. The function $L$ is the classical Lagrangian of the system whose quantum mechanical behaviour is to be described, and $S$ the corresponding classical Action.

In the following, (2.9) is not used per se to calculate the entire probability amplitude but rather the individual process amplitudes, that are combined according to Feynman's second principle, to obtain the complete path amplitude for the experiment under discussion. The most important of these process amplitudes is the Green function, or space-time propagator, for an on-shell photon or an excited atom. These are derived in the following section, the former from (2.9) and the latter, more conveniently, from the time-dependent Schrödinger equation, shown by Feynman $[6,7]$ to be equivalent, in the non-relativistic limit, to (2.9). In his book on quantum mechanics Dirac used, conversely, the Schrödinger equation to derive (2.9) [19].

The physical interpretation of Feynman's principles I-V will now be discussed. In typical treatments of 'The Interpretation of Quantum Mechanics' in the literature it is customary to attempt to reduce the problem to its bare essentials by avoiding explicit reference to any actual application. The physical meaning of Hilbert space vectors, or wavefunctions, is discussed in complete generality, the dynamical content being limited to the 'unitary evolution' of fixed energy solutions of the time-dependent Schrödinger equation. The danger of such a procedure is that of over-simplification. Instead of interpreting how quantum mechanics describes actual experiments, what is done is to instead 'interpret' a simple mathematical model, that does not necessarily reflect the actual complexity of a real experiment. To avoid this danger here, the discussion focuses specifically on the type of experiment treated in [1]:

(i) An excited atom is created.

(ii) The atom decays spontaneously, emitting a single photon.

(iii) The photon interacts with the experimental apparatus.

(iv) A photon (not necessarily the decay photon) is detected.

\footnotetext{
${ }^{7}$ Here $\vec{x}_{p} \equiv\left(x_{p}^{1}, x_{p}^{2}, x_{p}^{3}\right)$ and the dot denotes a time derivative
} 
First consider the simpler sequence (i), (ii) and (iv) only. The atom is created, it decays, and the photon propagates through free space to the detector. If the detector has a surface area, $S$, perpendicular to the space vector, $\vec{r}$, drawn from the excited atom to the detector, and the atom is unpolarised, there is no need to use quantum mechanics (or classical mechanics either) to calculate the time-integrated probability that the photon will be detected. All that is needed is a knowledge three-dimensional spatial geometry. The answer is: $\epsilon_{D} S /\left(4 \pi r^{2}\right)$, where $\epsilon_{D}$ is the efficiency of the photon detector. In order to calculate the probabilty, $\delta P\left(t_{D}\right)$, that the photon will be detected in the time interval $\delta t_{D}$ at $t_{D}$, quantum mechanics is needed, but only to calculate the mean lifetime, $\tau_{S}$, of the excited source atom. Suppose that the excited atom is produced by the passage of a pulsed laser beam tuned to the frequency $\left(E_{i}-E_{f}\right) / h$ where $E_{i}$ and $E_{f}$ are the excited and ground state atomic energy levels. The laser pulse is assumed to have a Gaussian form with variance $\sigma_{t}$. If the mean time of passage of the exciting pulse is $t_{0}$ and the production time of the excited atom is $t_{P}$, the above-mentioned probability is:

$$
\begin{aligned}
\delta P\left(t_{D}\right) & =\sqrt{\frac{2}{\pi}}\left(\frac{\epsilon_{D} S}{4 \pi r^{2}}\right) \frac{\sigma_{t}}{\tau_{S}} \int_{-\infty}^{\infty} \exp \left[-\frac{1}{\tau_{S}}\left(t_{D}-t_{P}-\frac{r}{c}\right)-\frac{\left(t_{P}-t_{0}\right)^{2}}{2 \sigma_{t}^{2}}\right] d t_{P} \delta t_{D} \\
& =\frac{\epsilon_{D} S}{4 \pi \tau_{S} r^{2}} \exp \left[-\frac{1}{\tau_{S}}\left(t_{D}-t_{0}-\frac{r}{c}-\frac{\sigma_{t}^{2}}{2 \tau_{S}}\right)\right] \delta t_{D}
\end{aligned}
$$

The only physical postulates necessary to derive this result are an exponental decay law for the excited atom and a constant velocity, c, for the decay photon.

It will now be instructive to re-derive this result using Feynman's principles. The corresponding path amplitude is, using II:

$$
P A_{f i}=A_{D}\langle D|\gamma| \gamma\rangle\left\langle\gamma|T| A_{i}\right\rangle\left\langle\gamma\left|A_{i}\right| P\right\rangle A_{P}
$$

The five process amplitudes have the following meaning:

- $A_{P}$ is the production amplitude for process (i) above.

- $\left\langle\gamma\left|A_{i}\right| P\right\rangle^{8}$ is the process amplitude for the propagation of the excited atom from its production time $t_{P}$ to its decay time $t_{\gamma}$ (see (3.20) below):

$$
\left\langle\gamma\left|A_{i}\right| P\right\rangle=\exp \left[-\frac{i}{\hbar}\left(E_{i}^{0}-E_{f}^{0}-i \frac{\hbar}{2 \tau_{S}}\right)\left(t_{\gamma}-t_{P}\right)\right]
$$

$E_{i}^{0}$ and $E_{f}^{0}$ are the 'pole center-of-mass energies' (see Section 3 below) of the atomic states $i$ and $f$, and $\tau_{S}$ is the mean lifetime of the state $i$.

- $\left\langle\gamma|T| A_{i}\right\rangle$ is the invariant amplitude for the atomic transition: $A_{i} \rightarrow A_{f}+\gamma$.

- $\langle D|\gamma| \gamma\rangle$ is the propagator of the photon from its production time $t_{\gamma}$ to its detection time $t_{D}$ (see (3.11) below):

$$
\langle D|\gamma| \gamma\rangle=\frac{1}{r} \exp \left[-\frac{i}{\hbar}\left[\left(E_{\gamma}\left(t_{D}-t_{\gamma}\right)-p_{\gamma} r\right]\right]=\frac{1}{r}\right.
$$

The last member of (2.13) follows since the photon is a massless particle with constant velocity $c: c=E_{\gamma} / p_{\gamma}=r /\left(t_{D}-t_{\gamma}\right)$.

\footnotetext{
${ }^{8}$ The notation used for space-time propagators is that $\langle f|P| i\rangle$ is the amplitude for a particle (or other quantum object, such as an atom or molecule), $P$, initially at space-time point $x_{i}$, to be found at $x_{f}$.
} 
- $A_{D}$ is the detection amplitude for process (iv) above.

Also required for the calculation is the density distribution, $\rho_{P}$, for production of the excited atom and the density, $\rho_{D}$ of detected final states:

$$
\begin{aligned}
d \rho_{P} & =\sqrt{\frac{2}{\pi}} \sigma_{t} \exp \left[-\frac{\left(t_{P}-t_{0}\right)^{2}}{2 \sigma_{t}^{2}}\right] d t_{P} \\
\rho_{D} & =\delta t_{D}
\end{aligned}
$$

Using I, noting that $t_{\gamma}=t_{D}-r / c$, and integrating over $t_{P}$ gives:

$$
\begin{aligned}
\delta P\left(t_{D}\right)^{P A} & =\int\left|P A_{f i}\right|^{2} d \rho_{P} \delta t_{D} \\
& =\sqrt{\frac{2}{\pi}} \sigma_{t} \frac{A_{0}^{2}}{r^{2}} \int_{-\infty}^{\infty} \exp \left[-\frac{1}{\tau_{S}}\left(t_{D}-t_{P}-\frac{r}{c}\right)-\frac{\left(t_{P}-t_{0}\right)^{2}}{2 \sigma_{t}^{2}}\right] d t_{P} \delta t_{D} \\
& =\frac{A_{0}^{2}}{r^{2}} \exp \left[-\frac{1}{\tau_{S}}\left(t_{D}-t_{0}-\frac{r}{c}-\frac{\sigma_{t}^{2}}{2 \tau_{S}}\right)\right] \delta t_{D}
\end{aligned}
$$

where

$$
A_{0}^{2} \equiv\left|A_{D}\right|^{2}\left|\left\langle\gamma|T| A_{i}\right\rangle\right|^{2}\left|\left\langle\gamma|T| A_{i}\right\rangle\right|^{2}\left|A_{P}\right|^{2}
$$

Comparing with the (2.10) shows that, for consistency:

$$
\left|A_{D}\right|^{2}\left|\left\langle\gamma|T| A_{i}\right\rangle\right|^{2}\left|\left\langle\gamma|T| A_{i}\right\rangle\right|^{2}\left|A_{P}\right|^{2}=\frac{\epsilon_{D} S}{4 \pi \tau_{S}}
$$

This is perfectly reasonable since $\left|\left\langle\gamma|T| A_{i}\right\rangle\right|^{2} \simeq \Gamma_{S}=\hbar / \tau_{S}$, and $\left|A_{D}\right|^{2} \simeq S$ so that the photon detection probability is proportional to the area of the detector.

The above comparison has shown the consistency of the calculation based on Feynman's quantum mechanical principles with the 'common sense' result (2.10). This example will now be compared with the typical description of the free space propagation of particles to be found in text books on quantum mechanics, quantum field theory, or optics. For example, Dirac writes down the wavefunction of a free particle in an energy-momentum eigenstate as [20]:

$$
\psi(x y z t)=a_{0} \exp \left[-i \frac{p \cdot x}{\hbar}\right]=a_{0} \exp \left[-\frac{i}{\hbar}\left(E t-p_{x} x-p_{y} y-p_{z} z\right)\right]
$$

where $x=(c t, \vec{x})$ and $p=(E / c, \vec{p})$ are the space-time and energy-momentum 4-vectors of the particle. Dirac then states that such a 'plane wave' wavefunction has no physical significance, since it predicts the particle to have the same probability to be at any point in space-time ${ }^{9}$. A similar description the photon in the above example would be a radial wave function of the form $\exp [-i p \cdot x / \hbar] / r$ which would predict that the photon has the same probability to be found at any distance from the source at any time. Such 'wavefunctions' are evidently meaningless and bear no relation to the actual physical situation correctly described by (2.10) or (2.16). Dirac indeed agrees that such a wavefunction is meaningless, but suggests that the solution to the problem is to replace the plane wave in (2.18) by

\footnotetext{
${ }^{9}$ Actually, since the wavefunction of (2.18) is not square-integrable, the normalisation constant $a_{0}$ vanishes, as does also the probability that the particle is within any finite volume of space-time
} 
a spatial 'wave packet'. This is then claimed to give a correct quantum description of actual free particles. It is argued that since the plane wave in (2.18) is an eigenstate of $E$ and $p$, that it is natural from the Heisenberg Uncertainty Relations: $\Delta p \Delta x \geq \hbar$ and $\Delta E \Delta t \geq \hbar$ that that both $x$ and $t$ should be completely uncertain. Since it is certainly true that actual particles are never produced in an eigenstate of $E$ and $p$ it is further argued that they must therefore be produced with spatial wavepackets that are coherent superpositions of different $E, p$ eigenstates. Indeed the photons produced by the decay of the excited atom in the above example do have an energy distribution of non-vanishing width. Nevertheless, there is no associated spatial wavepacket. The absurdity of describing the photon in the above example by a wave packet of the type suggested by Dirac, becomes evident on examining the physical consequences of such a hypothesis. Suppose that $\tau_{S}$ has the typical value of $10^{-8} \mathrm{sec}$. The correponding spread, $\Delta p$, in the momentum of the photon is given correctly by the energy-time Uncertainty relation as $c \Delta p=\Delta E \simeq \Gamma_{S}=\hbar / \tau_{S}{ }^{10}$. According the the momentum-space Uncertainty Relation invoked by Dirac, the width, $\Delta x$, of the associated spatial wavepacket should be: $\Delta x \simeq \hbar / \Delta p \simeq c \tau_{S} \simeq 3 \mathrm{~m}$. If such a wavepacket were actually produced in the decay of the excited atom in the above example, quantum mechanics would predict that it could be instantaneously detected at a distance of, say, $1 \mathrm{~m}$ from the production point, in contradiction to the predictions of (2.10) and (2.16) above. Using, say, a photo-diode to detect the photon, in the above example, its spatial position is easily measured with a precision of $\Delta x \simeq 1 \mathrm{~mm}$. This implies that, at the moment of detection, the product $\Delta x \Delta p$ for the photon is known with an accuracy $3 \times 10^{3}$ better than allowed by the Uncertainty Principle. The latter is evidently not applicable in this case.

The point is that the relation $\Delta x \Delta p \geq \hbar$ applies either to simultaneous measurements of $x$ and $p$, as in the examples orginally discussed by Heisenberg [21], or to the characteristic widths of the spatial and momentum distributions of a bound state wavefunction, or the wave packets which correctly describe a beam of massive particles such as electrons or neutrons. These distributions are related by a Fourier transform, from which the corresponding Uncertainty Relation is easily derived. In the example above, knowledge of the excited state of the atom provides prior knowledge of the momentum uncertainty of the photon as $\hbar /\left(c \tau_{S}\right)$ where $\tau_{S}$ is the previously measured or theoretically calculated mean life of the excited atom. No measurement is required to know the photon momentum with this accuracy ${ }^{11}$

What is not taken into account in Dirac's discussion, and that of other text book authors is that, in quantum mechanics, real ('on-shell') particles propagate over macroscopic distances in a classical manner. This fact is taken fully into account in the Feynman space-time formulation of quantum mechanics, and, as will be demonstrated in the examples worked out later in the present paper, is crucial in deriving its predictions. As will be seen, many of the latter are identical to those of the classical wave theory of light, that are well verified experimentally. The conclusion is that the position of a particle that has already been produced must be defined in a classical manner in order to correctly calculate the correponding path amplitude. The energy-time Uncertainty Relation $\Gamma_{S} \tau_{S}=\hbar$

\footnotetext{
${ }^{10}$ It is here assumed that the lower energy level of the atomic transition that produces the photon is the ground state, with infinite lifetime

${ }^{11}$ Here incoherent line broadening effects due, for example, to the Doppler effect, are neglected, so the atom is supposed to be isolated and at low temperature.
} 
of course plays an essential role in the derivation of the common sense equation (2.10): there is quantum uncertainty in the time of decay. Once a photon is produced, however, it propagates over macroscopic distances according to the classical law: $\Delta r / \Delta t=c$ within each path amplitude. Adding the path amplitudes to construct the probability amplitude modifies, via quantum interference, this simple behaviour, and gives rise to the purely quantum effects of refraction, diffraction and interference that will be discussed below.

It is possible to replace Dirac's wavefunction (2.18) by one which does give a meaningful space-time description of the photon produced in the decay of the excited atom. Since the detection process can be considered as a probe of the spatial position of the photon, the 'photon wavefunction' can be defined by omitting the amplitude $A_{D}$ in (2.11) and multiplying by a suitable $\delta$-function to describe the classical space-time propagation property of the photon. Thus the radial wavefunction of the photon is given by (2.11)-(2.13) as:

$$
\psi\left(\vec{r}, t, t_{\gamma}, t_{P}\right)=\frac{a_{0}}{r} \exp \left[-\frac{i}{\hbar}\left(E_{i}^{0}-E_{f}^{0}-\frac{i \hbar}{2 \tau_{S}}\right)\left(t-t_{P}-\frac{r}{c}\right)\right] \delta\left(r-c\left(t-t_{\gamma}\right)\right)
$$

The wavefunction of the photon depends not only on the spatial position and the time as specified by $\vec{r}$ and $t$, but also on the time at which it is produced $t_{\gamma}$, as well as the production time $t_{P}$ of the excited atom. Using the Born probabilty rule:

$$
P=\int|\psi|^{2} d V
$$

and the density distributions (2.14) and (2.15), the wavefunction of (2.19) is easily seen to give, with a suitable choice of the normalistation constant $a_{0}$, the same result for $\delta P(t)$ as (2.10) or (2.16).

The reader may now be asking why, since the common sense formula (2.10) already describes the probability to observe the decay photon at any time, assuming only an exponential decay law and that the photon is a massless particle, what is the use of Feynman's formulation of quantum mechanics (that gives the same prediction via a more complicated calculation) or indeed of quantum mechanics in any formulation, except for calculating the mean lifetime of the excited atom? The answer lies in the description of space-time experiments where principle III (quantum mechanical superposition) plays a crucial role. This is the case in all the examples worked out in the present paper. The essential information provided by the path amplitude concerns not the spatial position of the particle (which is calculated classically) but the phase of each path amplitude at the instant the final state is measured. It is these phases which control the observed quantum interference phenomena. However, the particle does move in a classical manner along each path. As Feynman strongly emphasised, the difference between a classical world and the actual quantum one is that the different paths in one-to-one correspondence with different classical histories of the quantum system contribute together, in parallel in time, according to (2.3), to the probability amplitude that describes a single detection event. This effect is most simply and graphically illustrated by the Young double-slit interference experiment, to be analysed in detail in Section 9 below, that Feynman chose to exemplify this behaviour [22], but in reality not two, but an infinite number of paths each with its own distinct classical history, are combined, in parallel, when quantum mechanical superposition operates. 
It is interesting to note that the actual physical description provided by Feynman's formulation of quantum mechanics contains important elements of several different socalled 'interpretations' of quantum mechanics. These are:

(a) 'Consistent Histories'

(b) Everett's 'Many Worlds' interpretation

(c) de Broglie's original 'Pilot Wave' theory

Each of these interpretations is now briefly discussed in comparison with Feynman's formulation.

'Consistent Histories' [23, 24, 25] are usually presented in the language of Hilbert space projection and trace operators. In the notation of the present paper, and considering the simplest non-trivial case, with a single intermediate quantum 'property' [24], consistent histories are given by summed path amplitudes similar to those in (2.3) above, except that the sum over intermediate states is not over the complete set allowed by a given experimental configuration, as is understood in (2.3), but is instead limited to an arbitary domain, $D_{k}$. Probabilities are assigned these sets of paths according to a definition similar to Feynman's first principle, (2.1):

$$
\begin{aligned}
P_{f i}\left(D_{k}\right) & =\left|A_{f i}\left(D_{k}\right)\right|^{2} \\
P_{f i}^{G}\left(D_{k}\right) & =\frac{\left|A_{f i}\left(D_{k}\right)\right|^{2}}{\left|A_{f i}\right|^{2}}
\end{aligned}
$$

where

$$
A_{f i}\left(D_{k}\right) \equiv \sum_{D_{k}}\left\langle f\left|T_{f}\right| k\right\rangle\left\langle k\left|T_{k}\right| i\right\rangle
$$

(2.20) corresponds to the history definition of Omnès [24] and Gell-Mann and Zurek [25], whereas (2.21) is the original definition due to Griffiths [23]. The amplitude $A_{f i}$ in the denominator of (2.21) is given by replacing the limited domain $D_{k}$ of (2.22) with the complete set of allowed intermediate states as understood in (2.3). Histories defined by different domains $D_{k}^{\prime}, D_{k}^{\prime \prime}$ are said to be 'consistent' if the probabilites calculated according to $(2.20)$ or (2.21) add in a classical manner:

$$
P_{f i}\left(D_{k}^{\prime}+D_{k}^{\prime \prime}\right)=P_{f i}\left(D_{k}^{\prime}\right)+P_{f i}\left(D_{k}^{\prime \prime}\right)
$$

Conditions for this to be true were first given in the projection operator and trace formalism by Griffiths [23]. They are equivalent to requiring that the interference terms between the corresponding amplitudes $A_{f i}\left(D_{k}^{\prime}\right)$ and $A_{f i}\left(D_{k}^{\prime \prime}\right)$ vanish:

$$
\operatorname{Re}\left[A_{f i}\left(D_{k}^{\prime}\right) A_{f i}\left(D_{k}^{\prime \prime}\right)^{*}\right]=0
$$

This condition is satisfied if the difference between the phases of $A_{f i}\left(D_{k}^{\prime}\right)$ and $A_{f i}\left(D_{k}^{\prime \prime}\right)$ is an odd multiple of $\pi / 2$, i.e. if the corresponding vectors in the complex plane are orthogonal. The 'consistent' histories are then those for which the quantum interference effects resulting from superposition vanish. The 'histories' associated with the Feynman path amplitudes that describe actual experiments have, of course, no such restriction. 
The relation of Feynman's formulation of quantum mechanics to Everett's 'Many Worlds' or 'Relative State' formulation, can be seen by comparing their descriptions of the Young double slit interference experiment. Suppose that, initially, photon detectors are placed immediately behind the two slits and an observer (with a lot of time at his disposal!) records the responses of the detectors when single excited atoms are created nearby as a photon source. The observer will either note (mostly) that no photon is detected (0) or, more rarely, that a photon is detected either behind Slit 1 (1) or Slit 2 (2). Then, as suggested by Everett, the results recorded by the observer in sucessive repetitions of the experiment can be stored as a string of numbers: e.g. 00010020001... . Another observer performing a similar series of experiments might see another sequence: $00002000100 \ldots$. For a finite number of repetitions of the experiment there are a countable finite number of such sequences, each of which, Everett said correponds to a different possible 'history of the world', which will be hard-wired into the brain of the observer, or his recording device, at the end of his sequence of measurements. The actual history of any given observer is limited to just one such sequence, but all of the others describe 'alternative worlds' each with the same weight as the one actually observed..

Consider now the simplest case (unlikely but not impossible) when the observer in the first world notes a photon behind Slit 1 in his first experiment whereas the observer in the second world notes a photon behind Slit 2. Suppose now that the detectors are removed from behind the slits and placed in a position where a photon passing through either slit may be detected by them. There is clearly now a correspondence between the path amplitude for the photon to pass through Slit 1 and the 'world' of the first observer, and the path amplitude for the photon to pass through Slit 2 and the 'world' of the second observer ${ }^{12}$. Thus in a certain sense Everett's two possible 'worlds' are simultaneously present when the corresponding path amplitudes are added to give the probability amplitude for the Young double slit experiment. Since in any actual experiment an infinite number of different path amplitudes contribute to the probability amplitude it is as though an infinite number of classically distinct 'worlds' contribute, in parallel, to the probability amplitude. This is just what Feynman called the only real deep mystery of quantum mechanics.

In the early days of quantum mechanics, de Broglie ${ }^{13}$ proposed an interpretation called, in French 'La Théorie de la Double Solution' but usually now refered to as the 'Pilot Wave' formulation of quantum mechanics [27]. The idea was that the space-time coordinates of particles should appear explicitly in the theory so as to describe as, in classical mechanics, the 'real' positions of particles. There is also a purely mathematical, abstract, configuration-space wavefunction, $\psi$, associated with the particle which is conjectured to 'guide' the motion of the particle in such a way that that the statistical predictions of conventional quantum mechanics are recovered. This idea was taken up by Bohm and Hiley and developed into a fully-fledged deterministic alternative to non-relativistic quantum mechanics [28]. In this theory a 'quantum potential' is introduced that gives rise to classical forces that reproduce observed quantum behaviour.

\footnotetext{
${ }^{12}$ Photons passing through either slit do so whether or not there is a detector immediately behind it

${ }^{13} \mathrm{An}$ extensive discussion of these ideas can be found in [26]. In a later development of the theory the particle coordinates were replaced by those of a local singularity in a 'physical' wave, $u$, that was conjectured to exist in addition to the usual abstract configuration-space wavefunction, $\psi$. In this article de Broglie also imagined, like Feynman in [1], 'clocks' moving with the particles or waves and recording the phase information.
} 
However, as seen above, in Feynman's formulation, the space-time coordinates of particles do, in any case, occur in an essential way in the equations of the theory. The physical interpretation of the photon wavefunction in (2.19) is of a photon moving like a classical particle according to the law $\Delta r / \Delta t=c$, but also carrying with it a certain phase, analogous to de Broglie's 'Pilot Wave'. It is precisely this phase information, when combined with that of all other possible paths of the photon in any given experimental situation, that does in fact 'guide' the photon paths towards certain regions of space-time and away from others due to the effect of quantum interference. This process is exemplified in the calculations presented below in the present paper.

Two closing remarks in this section devoted to general principles. The first is that in the classical limit, $\hbar \rightarrow 0$, the sums over intermediate states in (2.3) reduce to a single term correponding to the classical path of the system. (2.1),(2.2) and (2.3) then give:

$$
\begin{aligned}
P_{f i}^{\text {Class }} & =\left|\left\langle f\left|T_{f}\right| k_{n}^{0}\right\rangle \ldots\left\langle k_{2}^{0}\left|T_{2}\right| k_{1}^{0}\right\rangle\left\langle k_{1}^{0}\left|T_{1}\right| i\right\rangle\right|^{2} \\
& =\left|\left\langle f\left|T_{f}\right| k_{n}^{0}\right\rangle\right|^{2} \ldots\left|\left\langle k_{2}^{0}\left|T_{2}\right| k_{1}^{0}\right\rangle\right|^{2}\left|\left\langle k_{1}^{0}\left|T_{1}\right| i\right\rangle\right|^{2} \\
& =P_{f n}^{0} \ldots P_{21}^{0} P_{1 i}^{0}
\end{aligned}
$$

Here $\left|k_{1}^{0}\right\rangle,\left|k_{2}^{0}\right\rangle \ldots$ are states along the classical path that minimises the action $S$ in (2.6). The last member of (2.25) is just a statement of Bayes Theorem ${ }^{14}$ for combining conditional probabilities in classical statistics.

Finally, a brief mention of the 'measurement problem' of quantum mechanics in relation to Feynman's formulation. It would seem, at first sight, that the traditional 'problems' of the 'reduction of the wavepacket' superposition of Hilbert space vectors of macroscopic states [30], with the associated 'Schrödinger cat' paradox etc. are completely avoided in Feynman's formulation. The essential theoretical concept, the probability amplitude is a complex number with a Lorentz invariant phase, which is completely and unambigously defined by Feynman's principles I-V and the experimental conditions that are to be described. On the other hand, Hilbert space state vectors are quite ambiguous, depending on the representation (Schrödinger , Heisenberg, Interaction,...) used to specify them. Such a Hilbert space vector, unlike Feynman's probability amplitude, does not describe the results of actual experiments. 'Measurements' in optics do not produce photons in quantum eigenstates, but rather destroy them. There is typically no 'wave packet' to contract, no superposition of Hilbert space vectors corresponding to different macroscopic final states of the 'detector', one probability amplitude for a live cat, a completely different one for a dead one... .

Feynman had very little respect for philosophers of science, and was too busy doing science to spend much time on such questions himself. This being so, the 'philosophy' of Feynman's formulation of quantum mechanics, unlike that of the Bohr, Heisenberg and Schrödinger one, still remains to be written.

\footnotetext{
${ }^{14}$ See, for example, [29].
} 


\section{Relativistic Path Integral and the Propagator of a Free On-Shell Particle}

A suitable starting point for the discussion is an integral equation for the one-dimensional path integral [31]:

$$
\psi(x, t+\epsilon)=\int_{-\infty}^{\infty} \frac{1}{A_{1}} \exp \left[\frac{i \epsilon}{\hbar} L\left(\frac{x-y}{\epsilon}\right)\right] \psi(y, t) d y
$$

where $\epsilon$ denotes a small time interval, $L$ is the relativistic Lagrangian of a free, on-shell, particle of pole mass, $m_{P}$, and relativistic velocity, $\beta=(x-y) /(c \epsilon)$, and the normalisation constant $A_{1}$ is to be determined by consistency in the limit $\epsilon \rightarrow 0, y \rightarrow x$. This formula is now generalised to three spatial dimensions by introducing the variable: $\vec{\eta}=\vec{x}-\vec{y}$, and changing the integration variable from $\vec{y}$ to $\vec{\eta}$ :

$$
\psi(\vec{x}, t+\epsilon)=\iiint \frac{1}{A_{3}} \exp \left[\frac{i \epsilon}{\hbar} L\left(\frac{|\vec{\eta}|}{\epsilon}\right)\right] \psi(\vec{x}-\vec{\eta}, t) d^{3} \eta
$$

Choosing the origin of coordinates such that $\vec{x}=(0,0,0)$, the one-axis parallel to the velocity vector of the particle: $\vec{\eta}=(\eta, 0,0)$ and making the replacement $t+\epsilon \rightarrow t$ gives:

$$
\psi(0,0,0, t)=\int d \eta_{2} \int d \eta_{3} \int_{0}^{\infty} \frac{1}{A_{3}} \exp \left[\frac{i \epsilon}{\hbar} L\left(\frac{\eta}{\epsilon}\right)\right] \psi(-\eta, 0,0, t-\epsilon) d \eta
$$

where, without loss of generality, $\eta$ has been chosen to be positive. The two and three axes are perpendicular to the direction of particle propagation. Since $\psi$ and $L$ on the right side of (3.2) do not depend on $\eta_{2}$ and $\eta_{3}$ the integral over these variables may be absorbed in the undetermined normalisation constant so that:

$$
\frac{1}{A} \equiv \frac{\int d \eta_{2} \int d \eta_{3}}{A_{3}}
$$

The relativistic free-particle classical Lagrangian is [32]:

$$
L\left(\frac{\eta}{\epsilon}\right)=-m_{P} c^{2} \sqrt{1-\frac{\eta^{2}}{c^{2} \epsilon^{2}}}=-m_{P} c^{2} \sqrt{1-\beta^{2}}
$$

Making a Taylor expansion in $\eta$ and $\epsilon$, of $\psi(-\eta, 0,0, t-\epsilon)$, retaining only the zeroth order term and changing the integration variable from $\eta$ to $\beta$ gives:

$$
\psi(0,0,0, t)=\int_{0}^{1} \frac{c \epsilon}{A} \exp \left[-\frac{i \epsilon}{\hbar} m_{P} c^{2} \sqrt{1-\beta^{2}}\right] \psi(0,0,0, t) d \beta
$$

Hence

$$
1=\int_{0}^{1} \frac{c \epsilon}{A} d \beta+O\left(\epsilon \eta, \epsilon^{2}\right)
$$

or, neglecting terms of $O\left(\epsilon \eta, \epsilon^{2}\right)$,

$$
A=c \epsilon
$$

Since

$$
\epsilon=\Delta t=\gamma \Delta \tau=\frac{\Delta \tau}{\sqrt{1-\beta^{2}}}
$$


where $t$ and $\tau$ are the laboratory and proper times respectively of the propagating particle, then, using (3.8) and (3.9), as well as the relation $c \epsilon=\eta / \beta,(3.3)$ may be written as:

$$
\begin{aligned}
\psi(0,0,0, t) & =\int_{0}^{\infty} \frac{\beta}{\eta} \exp \left[-\frac{i m_{P} c^{2}}{\hbar} \Delta \tau\right] \psi(-\eta, 0,0, t-\epsilon) d \eta \\
& =\int_{0}^{\infty} K(0,0,0, t ;-\eta, 0,0, t-\Delta t) \psi(-\eta, 0,0, t-\Delta t) d \eta
\end{aligned}
$$

where $K\left(\overrightarrow{x_{2}}, t_{2} ; \overrightarrow{x_{1}}, t_{1}\right)$ is the covariant Feynman propagator of the particle between the space time points $\left(\overrightarrow{x_{1}}, t_{1}\right)$ and $\left(\overrightarrow{x_{2}}, t_{2}\right)$. Exploiting the Lorentz invariance of the phase of the exponential in (3.10) the covariant propagator of a free, on-shell, particle may finally be written as:

$$
\begin{aligned}
K\left(\overrightarrow{x_{2}}, t_{2} ; \overrightarrow{x_{1}}, t_{1}\right) & =\frac{\beta}{\left|\overrightarrow{x_{2}}-\overrightarrow{x_{1}}\right|} \exp \left[-\frac{i m_{P} c^{2}}{\hbar} \Delta \tau\right] \\
& =\frac{\beta}{\left|\overrightarrow{x_{2}}-\overrightarrow{x_{1}}\right|} \exp \left[-\frac{i}{\hbar}\left(E\left(t_{2}-t_{1}\right)-\vec{p} \cdot\left(\overrightarrow{x_{2}}-\overrightarrow{x_{1}}\right)\right]\right.
\end{aligned}
$$

The propagator then has the form of a spherical wave, familiar from physical optics. Although, in the derivation, the spatial interval $\eta$ was assumed to be a 'small' quantity it should be noted that there is no length scale in the propagator of a free on-shell particle. In any case, Huygen's construction which, as will be demonstrated in the following section, is recovered in the path amplitude formalism, guarantees spherical wave propagation from the combination of, in principle, infinitely short spherical 'wavelets' [33]. Equation (3.11) is therefore of general validity.

The mass parameter, $m_{P}$, in (3.11) is, by definition, the 'pole mass' of the particle. In the case that the latter is unstable, with mean lifetime $\tau_{M},(3.11)$ is modified to:

$$
K\left(\overrightarrow{x_{2}}, t_{2} ; \overrightarrow{x_{1}}, t_{1}\right)=\frac{\beta}{\left|\overrightarrow{x_{2}}-\overrightarrow{x_{1}}\right|} \exp \left[-\frac{i c^{2}}{\hbar}\left(m_{P}-\frac{i \Gamma}{2 c^{2}}\right) \Delta \tau\right]
$$

where the decay width $\Gamma \equiv \hbar / \tau_{M}$ has been introduced. The physical mass, $m$, of the particle is then smeared around the value $m_{P}$ according to a distribution of width $\simeq \Gamma / c^{2}$. This distribution (the momentum-energy space propagator of the unstable particle) is given by the Fourier transform with respect to $\Delta \tau$ of the exponential factor in (3.12):

$$
K\left(E ; E_{0}, \Gamma\right)=\int_{0}^{\infty} \exp \left[-\frac{i}{\hbar}\left(E_{0}-\frac{i \Gamma}{2}\right) \Delta \tau\right] \exp \left(\frac{i E \Delta \tau}{\hbar}\right) d(\Delta \tau)
$$

where $E_{0} \equiv m_{P} c^{2}$ is the center-of-mass energy corresponding to the pole mass and $E \equiv$ $m c^{2}$ is the physical center-of-mass energy of the particle. The integral in (3.13) yields:

$$
K\left(E ; E_{0}, \Gamma\right)=\frac{\hbar}{i\left(E-E_{0}\right)-\Gamma / 2}
$$

This propagator corresponds to the well-known Lorentzian 'natural' optical line shape in the case that the 'particle' is identified with the initial atomic state in a spontaneous radiative decay process. For the decay of an unstable elementary particle it is the equally familiar Breit-Wigner amplitude. 
It will be important, for the discussion of the damping of interference effects in Section 8 below, to note that the imaginary part of the argument of the exponential in the spacetime propagator (3.12) is a function of the pole mass, not the physical mass, of the particle.

The dimension, $\left[L^{-1}\right]$, of the space-time propagator is a necessary consequence of its definition in (3.10). Although the phase of this amplitude is Lorentz-invariant, the entire amplitude is not. The propagator may also be written as:

$$
K\left(\Delta \tau, m_{P}, \gamma\right)=\frac{1}{c \gamma \Delta \tau} \exp \left[-\frac{i m_{P} c^{2}}{\hbar} \Delta \tau\right]
$$

where the appearence of the relativistic parameter $\gamma$ makes manifest the non-Lorentzinvariant character of the propagator. In the rest frame of the particle, (3.15) becomes:

$$
K\left(\Delta \tau, m_{P}, 1\right)=\frac{1}{c \Delta \tau} \exp \left[-\frac{i m_{P} c^{2}}{\hbar} \Delta \tau\right]
$$

which differs from Feynman's [34] asymptotic Lorentz-invariant space-time propagator:

$$
K^{a s y m}\left(\Delta \tau, m_{P}\right)=\frac{m_{P}}{8 \pi i c \Delta \tau} \exp \left[-\frac{i m_{P} c^{2}}{\hbar} \Delta \tau\right]
$$

only by the factor $m_{P}$ and a normalisation constant. Neither (3.16) nor (3.17) is suitable to describe the time evolution of the wavefunction of a particle at rest, due to the manifestly unphysical behaviour of the factor $(c \Delta \tau)^{-1}$ originating in the spatial integral in (3.10) which is no longer defined for a particle at rest. In seems that the one dimensional propagator describing the time evolution of the state of a particle at rest can not be obtained as a simple limit of the four-dimensional space-time propagator. Instead, the propagator of a particle at rest may be simply and directly derived from the timedependent Schrödinger Equation. For a particle at rest the non-relativistic limit necessary for the validity of the Schrödinger Equation is actually the same as the exact kinematical result, so that the propagator found in this way is both exact, and Lorentz-invariant. However the 'Schrödinger Equation' used here, though universally so-called in discussions of particle flavour oscillations, is in fact a relativistic version of this equation since the non-relativistic kinetic energy term of the conventional equation is replaced by the sum of this term and the rest energy of the particle. In the use of such an equation to describe atomic transitions the rest mass term contributes an overall multiplicative phase factor in the solutions of the equation, without any physical consequences, since this phase factor cancels in all atomic transition matrix elements. In physical optics, as aleady mentioned above, the crucial dynamical element in the probability amplitude is the temporal propagator of the excited atom that constitutes the photon source, or, more precisely, the time dependence of the decay amplitude of such an atom. Although this formula may be found, for example, in Dirac's book on quantum mechanics [35], for clarity and completeness it is rederived here.

Denoting the mass of the atomic ground state by $m_{0}$ and the pole excitation energy of the state $\left|\psi_{i}\right\rangle$ by $E_{i}^{0}$ the Schrödinger Equation for this quasi-stationary state is:

$$
i \hbar \frac{\partial\left|\psi_{i}\right\rangle}{\partial \tau}=\left(m_{0} c^{2}+E_{i}^{0}\right)\left|\psi_{i}\right\rangle
$$


with the solution:

$$
\left|\psi_{i}(\tau)\right\rangle=\left|\psi_{i}(0)\right\rangle \exp \left[-\frac{i\left(m_{0} c^{2}+E_{i}^{0}\right)}{\hbar} \tau\right]
$$

Hence the time evolution of the transition amplitude between the states $\left|\psi_{i}\right\rangle$ and $\left|\psi_{f}\right\rangle$ is give by the relation:

$$
\left\langle\psi_{f}(\tau)|T| \psi_{i}(\tau)\right\rangle=\left\langle\psi_{f}(0)|T| \psi_{i}(0)\right\rangle \exp \left[-\frac{i\left(E_{i}^{0}-E_{f}^{0}-i \Gamma_{i} / 2\right)}{\hbar} \tau\right]
$$

where an imaginary part $-i \Gamma_{i} / 2$ has been added to $E_{i}^{0}$ to take into account, as discussed above, the lifetime damping of the amplitude of the excited state. In path amplitude language, (3.20) may be interpreted as the product as the amplitude for the excited state to evolve from proper time zero to proper time $\tau$ (the exponential factor) times the time independent decay amplitude of the state. The formula (3.20) is directly applicable to nuclear $\beta$ transitions on making the relacement $E_{i}^{0}-E_{f}^{0} \rightarrow E_{\beta}^{0}$ where the latter quantity is the total energy release in the nuclear transition. As will be discussed in Section 9 below, the temporal propagator of the neutrino source state is predicted by the Feynman path amplitude formalism to make an important contribution to the neutrino oscillation phase. The time dependence of the decay amplitude of a particle that is destroyed in the transition is given by the replacements $E_{i}^{0} \rightarrow m_{P} c^{2}, E_{f}^{0} \rightarrow 0$, in (3.20) where $m_{P}$ is the pole mass of the particle. That is, the 'excitation energy' of the particle is equivalent to its mass, while the mass-energy of the corresponding 'ground state' vanishes.

\section{Derivation of the Classical Wave Theory of Light from the Path Amplitude Formalism Of Quantum Mechanics}

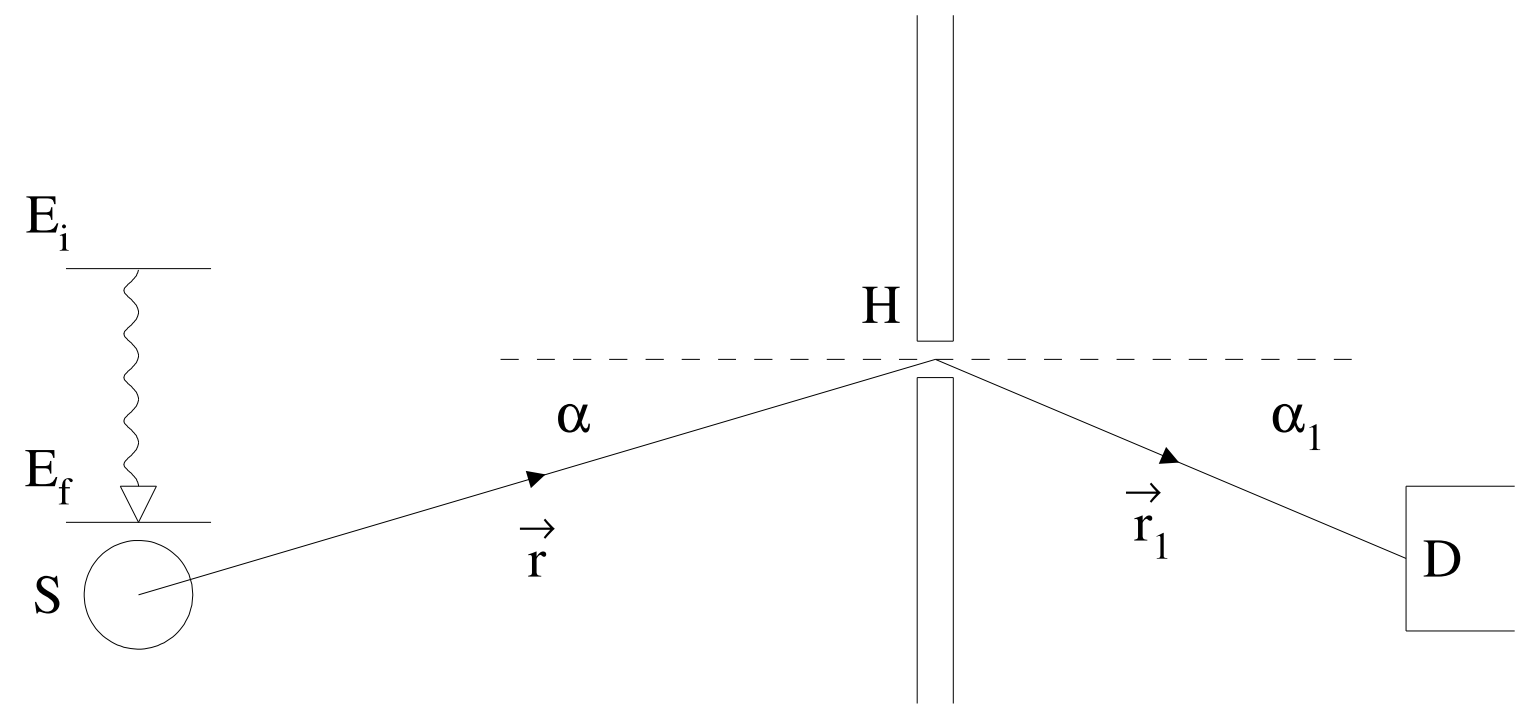

Figure 1: Diffraction of a photon, of energy $E_{i}-E_{f}$, produced in the spontaneous decay: $A_{i} \rightarrow A_{f}+\gamma$ of an excited atom at $S$, by a small hole, $H$, in an opaque screen. The photon is detected at $D$. The vectors $\vec{r}, \overrightarrow{r_{1}}$ lie in the plane of the figure 
The path amplitude $\left\langle f_{m}\left|k_{n}, \ldots k_{2}, k_{1}\right| i_{l}\right\rangle$ defined in (2.2) above is specified by the initial and final states $i_{l}$ and $f_{m}$ respectively, and by the set of unobserved intermediate states: $k_{n}, \ldots k_{2}, k_{1}$. The latter may refer to different space-time positions of the same particle, in which case, the 'process amplitude' $\left\langle k_{i}\left|T_{i}\right| k_{j}\right\rangle$ is the space-time propagator between $k_{j}$ : $\vec{x}_{j}, t_{j}$ and $k_{i}: \vec{x}_{i}, t_{j}$ or, alternatively, to the transition amplitude of some quantum process in which a particle is scattered or new particles are created. Of particular importance for the discussion of physical optics is the case of the creation of a photon in the spontaneous decay of an excited atom. In this case the first process amplitude (the rightmost one in (2.2)) describes the production of the excited atomic state, $\mathcal{A}_{i}$, from the ground state, $\mathcal{A}_{0}$, at time $t_{0}$ :

$$
\left\langle k_{1}\left|T_{1}\right| i_{l}\right\rangle=\left\langle\mathcal{A}_{i}\left|T_{P}\right| \mathcal{A}_{0}\right\rangle
$$

where $T_{P}$ denotes an atomic transition operator. The second process amplitude $\left\langle k_{2}\left|T_{2}\right| k_{1}\right\rangle$ describes the space-time propagation of the excited atom from its production time $t_{0}$, to the production time, $t_{\gamma}$ of the photon. The third process amplitude:

$$
\left\langle k_{3}\left|T_{3}\right| k_{2}\right\rangle=\left\langle\gamma \mathcal{A}_{f}|T| \mathcal{A}_{i}\right\rangle
$$

describes the decay of the excited atom into a photon, $\gamma$, of energy $E_{\gamma}=E_{i}-E_{f}$ where $E_{i}$ and $E_{f}$ denote the energies of the initial and final atomic states respectively. The appropriate formula to describe the product of process amplitudes $\left\langle k_{3}\left|T_{3}\right| k_{2}\right\rangle\left\langle k_{2}\left|T_{2}\right| k_{1}\right\rangle$, is given by (3.20):

$$
\left\langle k_{3}\left|T_{3}\right| k_{2}\right\rangle\left\langle k_{2}\left|T_{2}\right| k_{1}\right\rangle=\left\langle\gamma \mathcal{A}_{f}|T| \mathcal{A}_{i}\right\rangle \exp \left[-\frac{i}{\hbar}\left(E_{i}^{0}-E_{f}^{0}-i \Gamma_{i} / 2\right)\left(t_{\gamma}-t_{0}\right)\right]
$$

The first factor on both sides of (4.3) is the (time-independent) decay amplitude of the excited atom in (4.2). The second is the temporal propagator of the excited atom, assumed to be at rest.

The remaining product of process amplitudes $\left\langle k_{n}\left|T_{n}\right| k_{n-1}\right\rangle \ldots\left\langle k_{4}\left|T_{4}\right| k_{3}\right\rangle$ denote either free space propagation of photons according to (3.11) or, in the case of diffraction, refraction or reflection, photon scattering amplitudes. The final (left-most) process amplitude describes the photon detection process:

$$
\left\langle f_{m}\left|T_{f}\right| k_{n}\right\rangle=\left\langle\mathcal{D}_{m}\left|T_{D}\right| \mathcal{A}_{n} \gamma_{n}\right\rangle
$$

Here $\mathcal{D}_{m}$ denotes the final state of the photon detection process $\gamma_{n} \mathcal{A}_{n} \rightarrow \mathcal{D}_{m}$ that is the same in all the path amplitudes with final state label $m$. This may be an activated atom in a photographic plate, a photoelectron, a conduction electron in a photo-diode etc. The detection process does not discriminate the state $\gamma_{n}$ of the detected photon, and occurs at the same time, $t_{D}$, in all the path amplitudes with final state label $m$.

To illustrate the application of the path amplitude formula (2.2), a simple case is considered where an exited atom is produced at $\mathrm{S}$ and the decay photon is detected at $\mathrm{D}$ after diffraction by a small hole, $\mathrm{H}$, in an opaque screen (Fig. 1). The corresponding path amplitude is:

$$
\begin{aligned}
\Delta A= & \left\langle\mathcal{D}\left|T_{D}\right| \gamma\right\rangle\langle D|\gamma| H\rangle \mathcal{A}_{\text {diff }} \Delta S\langle H|\gamma| \gamma\rangle \\
& \left\langle\gamma \mathcal{A}_{f}|T| \mathcal{A}_{i}\right\rangle\left\langle\gamma\left|\mathcal{A}_{i}\right| 0\right\rangle\left\langle\mathcal{A}_{i}\left|T_{P}\right| \mathcal{A}_{0}\right\rangle
\end{aligned}
$$


The process amplitudes $\left\langle\gamma\left|\mathcal{A}_{i}\right| 0\right\rangle,\langle H|\gamma| \gamma\rangle$ and $\langle D|\gamma| H\rangle$ are, respectively, the space-time propagators of: the excited atom from its production time $t_{0}$ to its decay time $t_{\gamma}$, the photon, before diffraction at the hole at time $t_{H}$, and the photon after diffraction. The product of the three space-time independent process amplitudes, $\left\langle\mathcal{A}_{i}\left|T_{P}\right| \mathcal{A}_{0}\right\rangle,\left\langle\gamma \mathcal{A}_{f}|T| \mathcal{A}_{i}\right\rangle$ and $\left\langle\mathcal{D}\left|T_{D}\right| \gamma\right\rangle$ described above, is denoted below as $\tilde{\mathcal{A}}$. The diffractive scattering amplitude, $\mathcal{A}_{\text {diff }}$, will be derived below. The parameter $\Delta S$ is the area of the hole $\mathrm{H}$, which is used as a weighting factor proportional to the number of space time paths crossing the hole.

Since, for a massless photon, $E \Delta t-p \Delta x=m \Delta \tau=0$, there is no contribution to the phase of the path amplitude (4.5) from the photon propagators ${ }^{15}$. Using (4.2) and (4.3) the propagator of the excited atom is given s $^{16}$

$$
\left\langle P\left|\mathcal{A}_{i}\right| 0\right\rangle=\exp \left[-\frac{i}{\hbar}\left(E_{i}^{0}-E_{f}^{0}-i \Gamma_{i} / 2\right)\left(t_{\gamma}-t_{0}\right)\right]
$$

Noting now that

$$
t_{D}=t_{\gamma}+\frac{r+r_{1}}{c}
$$

the path amplitude of (4.5) may be written as :

$$
\Delta A=\frac{\tilde{\mathcal{A}}}{r r_{1}} \mathcal{A}_{d i f f} \Delta S \exp \left[-\frac{i}{\hbar}\left(E_{i}^{0}-E_{f}^{0}-i \frac{\Gamma_{i}}{2}\right)\left(t_{D}-t_{0}-\frac{r+r_{1}}{c}\right)\right]
$$

Here the factors $1 / r, 1 / r_{1}$ originate (see (3.11)) from the photon propagators.

Introducing the parameters:

$$
\kappa \equiv \frac{E_{i}^{0}-E_{f}^{0}}{\hbar c}=\frac{\left(M_{P}^{i}-M_{P}^{f}\right) c}{\hbar}
$$

and

$$
\rho \equiv \frac{\Gamma_{i}}{2 \hbar c}
$$

where $M_{P}^{i}$ and $M_{P}^{f}$ are the 'pole masses' of the initial and final atomic states, the path amplitude may be written as the product of four factors. The first has no spatio-temporal dependence, the second depends only on $r$, the third only on $r_{1}$ and the fourth only on the times $t_{D}$ and $t_{0}$ :

$$
\Delta A=\tilde{\mathcal{A}} \cdot \frac{e^{(i \kappa+\rho) r}}{r} \cdot \frac{e^{(i \kappa+\rho) r_{1}} \mathcal{A}_{d i f f} \Delta S}{r_{1}} \cdot e^{-c(i \kappa+\rho)\left(t_{D}-t_{0}\right)}
$$

In the description of diffraction or interference effects on the right of the screen in Fig. 1 , at a given detection time, $t_{D}$, and for sufficiently large values of $t_{D}-t_{0}$, only the $r_{1}$

\footnotetext{
${ }^{15}$ This was noted by Feynman on P103 of [1].'Once a photon has been emitted there is no further turning of the arrow as the photon goes from one point to another in space-time.'

${ }^{16}$ This is the physical origin of the 'stopwatch hand' discussed by Feynman in Fig 67 of [1]:'But when we construct a monochromatic source, we are making a device that has been carefully arranged so that the amplitude for a photon to be emitted at a certain time is easily caculated: it changes its angle at a constant speed like a stopwatch hand.' It is clear from (4.6) that the hand actually rotates in a clockwise direction, not an anticlockwise one as in Fig 67 of [1]. This change of sign has no effect on the predictions of the path amplitude calculations.
} 
variation of $\Delta A$ is of importance. If the finite decay width of the excited atom may also be neglected, i.e., if $\rho \ll \kappa$, the $r_{1}$ dependence of the path amplitude is that of a spherical wave field:

$$
U\left(r_{1}\right)=\frac{e^{i \kappa r_{1}}}{r_{1}}
$$

which satisfies the Helmholtz equation:

$$
\nabla^{2} U+\kappa^{2} U=0
$$

In this case, the description provided by the path amplitude formalism is mathematically identical to the classical wave theory of light. Diffraction is described by Kirchoff's equation [36, 37] and all the well-known interference phenomena of light, such as Huygen's construction [33], the Young double slit experiment and the Michelson Interferometer, may also be described purely spatially, like analogous wave phenomena of classical physics, such as transverse waves on a string or surface waves on a liquid subjected to the force of gravity. In coherent laser optics where the effective lifetime of the photon source is very long $^{17}, \rho \ll \kappa$, and so the classical wave theory of light is expected to be a very good approximation. It is therefore necessary to look elsewhere for the specifically temporal effects that distingish quantum mechanics from the classical wave theory.

The diffractive scattering amplitude, $\mathcal{A}_{\text {diff }}$, in (4.5) is derived by applying Green's Theorem, with suitable boundary conditions, to (4.13). This yields the well-known FresnelKirchoff diffraction formula [36], and the expression:

$$
\mathcal{A}_{\text {diff }}\left(\alpha, \alpha_{1}\right)=-\frac{i \kappa}{4 \pi}\left[\cos \alpha+\cos \alpha_{1}\right]
$$

where the angles $\alpha$ and $\alpha_{1}$ between the normal to the screen and the vectors $\vec{r}$ and $\overrightarrow{r_{1}}$ are shown in Fig 1. It is interesting to note that, in order to derive the FresnelKirchoff diffraction formula in the classical wave theory of light, it is necessary to assume, in addition to Green's theorem, that the wave field $U$ vanishes at large distances from the point under consideration. In the path amplitude description, this damping occurs automatically due to the factor: $\exp \left[-\rho\left(c\left(t_{D}-t_{0}\right)-r-r_{1}\right)\right]$ in (4.11). Since (see Fig.1) $c\left(t_{D}-t_{0}\right) \geq c\left(t_{D}-t_{\gamma}\right)=r+r_{1}$, the argument of the exponential factor is always zero or negative, producing strong damping of the amplitude for diffractive scattering at distances much larger than $r$ or $r_{1}$ since, in this case, $c\left(t_{D}-t_{0}\right) \geq c\left(t_{D}-t_{\gamma}\right) \gg r+r_{1}$.

The limit of the formula (4.14) for forward diffraction, can also be be derived, directly, using the path amplitude method. This is done by considering rectilinear photon progagation (propagation of plane waves in the classical theory of light). Consider a photon produced by a distant excited atom at $\mathrm{O}$ and observed in a photon detector at $\mathrm{D}$ as shown in Fig. 2. The $\mathrm{x}$-axis lies along the line joining the atom and the detector. Following Feynman [39] a plane surface perpendicular to the x-axis, distant $x$ from the atom and $x_{1}$ from the detector, where $x \gg x_{1}$, is imagined to be divided up into small areas, from each of which the photon trajectories from the atom may be diffracted towards the detector. In particular, diffraction is considered from a ring of radius $R_{1}$ and width $\Delta R_{1}$, centered on the x-axis. Using (4.8) above and noting that $c\left(t_{D}-t_{0}\right)-r-r_{1}=c\left(t_{\gamma}-t_{0}\right)$

$$
\Delta A=\frac{\tilde{\mathcal{A}}}{r r_{1}} \exp \left[-i \kappa\left(c\left(t_{D}-t_{0}\right)-r-r_{1}\right)-\rho c\left(t_{\gamma}-t_{0}\right)\right] \mathcal{A}_{d i f f} 2 \pi R_{1} \Delta R_{1}
$$

\footnotetext{
${ }^{17}$ Defining a 'coherence length': $L_{c o h}=c \hbar / \Gamma_{i}=1 /(2 \rho)$, typical values of $L_{c o h}$ for a spontaenously decaying atom and a coherent laser source are $3 \mathrm{~m}$ and $30 \mathrm{~km}$ respectively [38].
} 


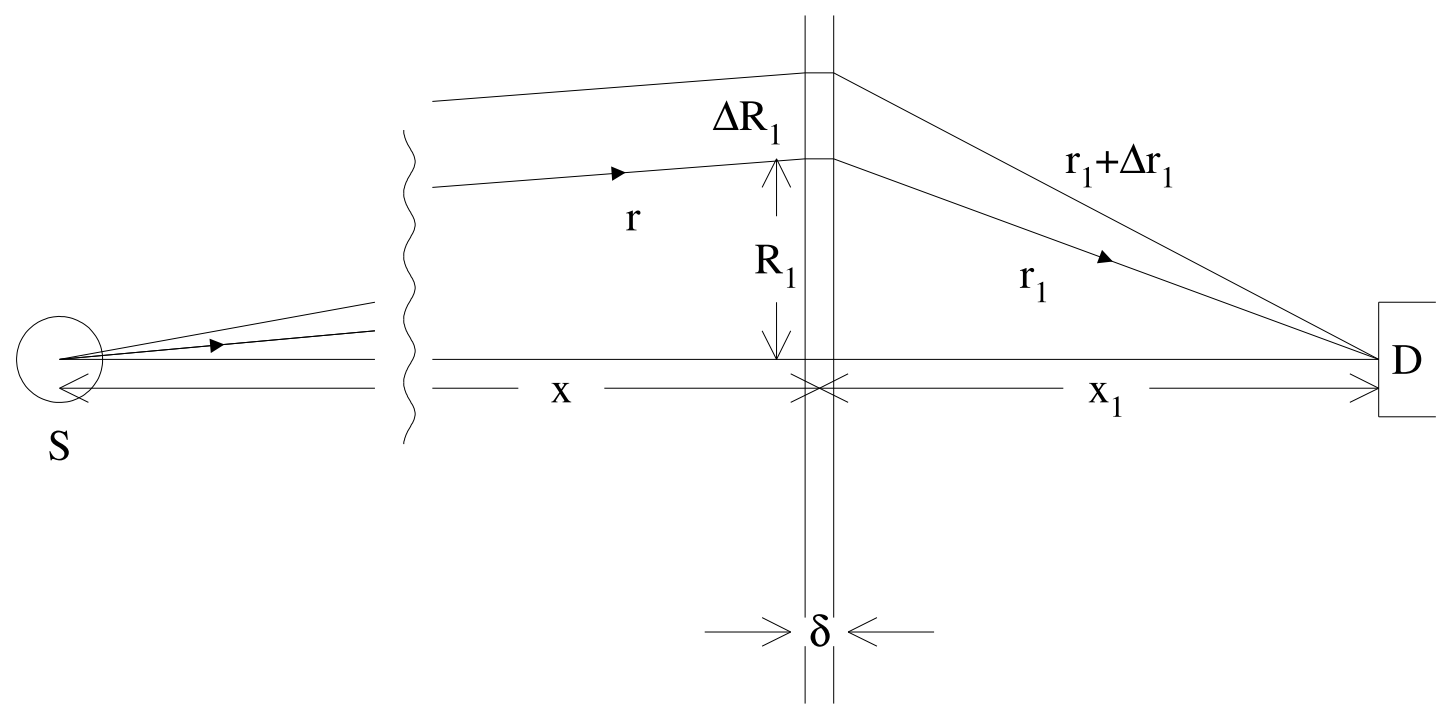

Figure 2: A photon produced by spontaneous decay of an atom at $S$ is detected at $D$. The integral of the path amplitudes of photons diffracted or scattered in a ring of width $\Delta R_{1}$ and radius $R_{1}$ in a plane perpendicular to $S D$ is constructed. In the case of diffraction, (4.16), the ring lies within an imaginary surface at distance $x$ from the source. In the case of scattering (refraction), (5.1), the ring lies in a thin sheet of transparent material of thickness $\delta$.

Since (see Fig. 2), $r_{1}^{2}=x_{1}^{2}+R_{1}^{2}$, it follows that $R_{1} \Delta R_{1}=r_{1} \Delta r_{1}$. Summing over all paths diffracted at the plane, the probability amplitude at the photon detector is:

$$
\left.A=\frac{\tilde{\mathcal{A}}}{r} \exp \left[-i \kappa\left(c\left(t_{D}-t_{0}\right)-r\right)\right] 2 \pi \mathcal{A}_{d i f f} \int_{x_{1}}^{r_{1}^{\max }} \exp \left[i \kappa r_{1}\right)-\rho c\left(t_{\gamma}-t_{0}\right)\right] d r_{1}
$$

where an arbitary upper limit, $r_{1}^{\max }$ has been assumed for the $r_{1}$ integral. Noting the inequality:

$$
c\left(t_{\gamma}-t_{0}\right) \geq r+r_{1}-x-x_{1}
$$

it can be seen that large values of $r_{1}$ will give an exponential suppression of the integrand in (4.16). In this case the $r_{1}$ integral is readily evaluated by use of the Huygens-Fresnel Principle [33]. This states that the value of the integral is one half of the contribution due to the first half-period zone. With the change of variable; $\varphi=\kappa r_{1}$, (4.16) may then be written as:

$$
\begin{aligned}
A & =\frac{\tilde{\mathcal{A}}}{r} \exp \left[-i \kappa\left(c\left(t_{D}-t_{0}\right)-r\right)-\rho c\left(t_{\gamma}-t_{0}\right)\right] \frac{\pi \mathcal{A}_{d i f f}}{\kappa} \int_{\kappa x_{1}}^{\kappa x_{1}+\pi} \exp [i \varphi] d \varphi \\
& =\frac{\tilde{\mathcal{A}}}{r} \exp \left[-i \kappa\left(c\left(t_{D}-t_{0}\right)-r-x_{1}\right)-\rho c\left(t_{\gamma}-t_{0}\right)\right]\left[\frac{2 \pi i \mathcal{A}_{\text {diff }}}{\kappa}\right] \\
& \simeq \frac{\tilde{\mathcal{A}}}{x_{S D}} \exp \left[-i \kappa\left(c\left(t_{D}-t_{0}\right)-x_{S D}\right)-\rho c\left(t_{\gamma}-t_{0}\right)\right]\left[\frac{2 \pi i \mathcal{A}_{d i f f}}{\kappa}\right]
\end{aligned}
$$

where, because of the small variation of $t_{\gamma}$ over the first half-period zone, the factor $\exp \left[-\rho c\left(t_{\gamma}-t_{0}\right)\right]$ has been taken outside the integral and in the last line, the approximation $r+x_{1} \simeq x+x_{1}=x_{S D}$ where $x_{S D}$ is the source-detector distance, has been made in the exponential factor and $r \simeq x_{S D}$ in the denominator. The path amplitude for photon 
propagation from the excited atom to the detector is given by an equation analogous to (4.5), with the fifth and sixth process amplitudes (in temporal order) omitted. The corresponding probability amplitude is:

$$
A=\frac{\tilde{\mathcal{A}}}{x_{S D}} \exp \left[-i \kappa\left(c\left(t_{D}-t_{0}\right)-x_{S D}\right)-\rho c\left(t_{\gamma}-t_{0}\right)\right]
$$

Consistency with (4.18) then requires:

$$
\mathcal{A}_{d i f f}=-\frac{i \kappa}{2 \pi}=-\frac{i}{\lambda_{\gamma}^{0}}
$$

where $\lambda_{\gamma}^{0}$ is the de Broglie wavelength of the photon ${ }^{18}$, in agreement with (4.14) for $\alpha=\alpha_{1}=0$. .

\section{The Refractive Index of a Uniform Transparent Medium}

The calculation of the diffractive scattering amplitude presented above is easily adapted to calculate the refractive index of a uniform transparent medium in terms of the atomic density, $\mathcal{N}$, and the elastic scattering amplitude, $\mathcal{A}_{\text {scat }}$, of a photon from an atom of the material. Thus, in Fig. 2, the plane in which the photons are considered to be diffracted is replaced by a thin, uniform, sheet of transparent medium of thickness $\delta$. Assuming an isotropic angular distribution for the scattered photons, the contribution to the path amplitude of photons scattering once from the atoms in the sheet ${ }^{19}$ is, by analogy with $(4.16)$, :

$$
A(\delta)=\frac{\tilde{\mathcal{A}}}{r} \exp \left[-i \kappa\left(c\left(t_{D}-t_{0}\right)-r-r_{1}\right)-\rho c\left(t_{\gamma}-t_{0}\right)\right] \mathcal{N} \mathcal{A}_{s c a t} \delta \int_{x_{1}}^{r_{1}^{\max }} \int_{0}^{2 \pi} d r_{1} d \phi_{1}
$$

where the integration variable has been changed from $R_{1}$ to $r_{1}$ and where the azimuthal angle, $\phi_{1}$, around the $\mathrm{x}$-axis, has been introduced. If the sheet is infinite in transverse extent the integral may be evaluated using the Huygens-Fresnel principle, as done for the case of diffraction above. As in all actual experiments the transparent material has finite transverse dimensions it will be found interesting, for the following discussion, to assume that the limit $r_{1}^{\max }$ is a function of a set of geometrical parameters, $d_{i}$, that define the transverse extent of the sheet, as well as of $\phi_{1}$. The examples of rectangular and circular

\footnotetext{
${ }^{18}$ Note that $\lambda_{\gamma}^{0}$ is defined, according to (4.9) and (4.20), in terms of the difference of the pole energies $E_{i}^{0}$ and $E_{f}^{0}$ of the atomic states $A_{i}$ and $A_{f}$, not their physical energies $E_{i}$ and $E_{f}$.

${ }^{19}$ Note a similar calculation of the radiation field from a 'sheet of oscillating charges' in Section 30-7 of [3], in particular the contribution of the upper limit of the $r$ integral. See also the analogous calculation of the refractive index of neutrons interacting in matter in Section 5.3.4 of [10].
} 
geometry are considered in Appendix A. In this case the equation analgous to (4.18) above is:

$$
\begin{aligned}
A(\delta)= & \left.\frac{\tilde{\mathcal{A}}}{r} \exp \left[-i \kappa\left(c\left(t_{D}-t_{0}\right)-r\right)\right)-\rho c\left(t_{\gamma}-t_{0}\right)\right] \frac{i \mathcal{N} \mathcal{A}_{\text {scat }} \delta}{\kappa} \\
& \times \int_{0}^{2 \pi}\left\{\left[\exp \left[i \kappa x_{1}\right]-\exp \left[i \kappa r_{1}^{\max }\left(d_{i}, \phi_{1}\right)\right]\right\} d \phi_{1}\right. \\
= & \left.\frac{\tilde{\mathcal{A}}}{x_{S D}} \exp \left[-i \kappa\left(c\left(t_{D}-t_{0}\right)-x_{S D}\right)\right)-\rho c\left(t_{\gamma}-t_{0}\right)\right] \frac{2 \pi i \mathcal{N} \mathcal{A}_{\text {scat }}}{\kappa} \delta
\end{aligned}
$$

since the $\phi_{1}$ average of $\exp \left[i \kappa r_{1}^{\max }\right]$ vanishes due to rapid phase variation. Adding the contribution from the path amplitudes of the unscattered photon from (4.19) gives:

$$
A^{\prime}=A+A(\delta)=A\left[1+\frac{2 \pi i \mathcal{N} \mathcal{A}_{s c a t} \delta}{\kappa}\right] \simeq A \exp [i \delta \phi]
$$

where

$$
\delta \phi=\frac{2 \pi \mathcal{N} \mathcal{A}_{\text {scat }} \delta}{\kappa}=\frac{2 \pi \mathcal{N} \mathcal{A}_{\text {scat }} f x}{\kappa}
$$

The last member of (5.3) is valid providing that $\delta$ is chosen sufficiently small that $\delta \phi \ll 1$. In (5.4) $f$ denotes the ratio $\delta /\left(x_{1}+x\right) \simeq \delta / x$, i.e. the fraction of the total distance between the exited source atom and the detector filled with the transparent material.

It is interesting to note that the result (5.2) may also be obtained by restricting the $r_{1}$ integral to the contribution of the first half-period zone. Thus the effect of random geometrical boundaries in a medium of finite spatial extent is the same as the use of the Huygens-Fresnel Principle in an infinite medium. This equivalence is used to simplify the calculation of the reflection coefficient at the surface of a transparent medium in Section 7 below.

Since the detection time $t_{D}$ and the production time $t_{0}$ of the excited atom are fixed, as is the distance between the photon source and the detector, the change of phase of the path amplitude induced by paths that scatter once on the atoms of the transparent material, implies that the apparent velocity, $v(f)$, of the light, between the source and the detector, when the fraction $f$ of the space between the source and the detector is filled with the transparent medium, is less than the speed of light in vacuum.

In vacuum:

$$
\phi(A)=-\kappa c\left(t_{D}-t_{0}-\frac{x}{c}\right)
$$

On adding the transparent material:

$$
\begin{aligned}
\phi\left(A^{\prime}\right) & =-\kappa c\left(t_{D}-t_{0}-\frac{x}{c}\right)+\delta \phi=-\kappa c\left(t_{D}-t_{0}-\frac{1}{c}\left(x+\frac{\delta \phi}{\kappa}\right)\right) \\
& =-\kappa c\left(t_{D}-t_{0}-\frac{x}{v(f)}\right)
\end{aligned}
$$

where, combining (5.4) and (5.6):

$$
\frac{c}{v(f)}=1+\frac{2 \pi \mathcal{N} \mathcal{A}_{\text {scatt }} f}{\kappa^{2}}
$$


Defining the refractive index of the transparent medium, $n$, as:

$$
n \equiv \frac{c}{v}=\frac{c}{v(1)}
$$

and using the last menber of (4.20), gives the well-known formula [40]:

$$
n=1+\frac{1}{2 \pi}\left(\lambda_{\gamma}^{0}\right)^{2} \mathcal{N} \mathcal{A}_{\text {scatt }}
$$

It is interesting to note that in the calculation, based on the the geometrical configuration shown in Fig.2, leading to (5.6), the photon corresponding to each individual path amplitude propagates at the vacuum speed $c$, but when the path amplitudes corresponding to single scattering processes are combined with those of unscattered photon the phase of the total amplitude is the same as that of a photon propagating in free space, but with a reduced velocity. This is clearly seen by inspection of (5.5) and (5.6) above. The apparent velocity, $v(f)$, has a linear dependence on the filling fraction $f$ :

$$
v(f)=(1-f) c+f v
$$

This equation may be re-arranged to give:

$$
\frac{\Delta v}{\Delta v_{\max }}=\frac{c-v(f)}{c-v}=f
$$

which states that the change of effective velocity normalised to its maximum value is equal to $f$, i.e. it is proportional to the number of scattering processes contributing to the probability amplitude.

It may be remarked that the above calculation of the refractive index has been carried out entirely in the language of particles moving in space-time. The de Broglie wavelength $\lambda_{\gamma}^{0}$ has been introduced in (5.9) only to show that the usual wave-mechanics result is recovered in the path amplitude approach.

The calculation of the refractive index just presented was performed on the assumption that the the sheet of transparent medium is sufficiently thin that the approximation of the last member of (5.3) is valid, and that only a single scattering event needs to be considered. Combining (5.4) and (5.9) the phase shift produced by the sheet is:

$$
\delta \phi=\frac{2 \pi(n-1) \delta}{\lambda_{\gamma}^{0}}
$$

Thus, for the approximation $1+i \delta \phi \simeq \exp i \delta \phi$ to be valid, $\delta$ is required to be a tiny fraction of $\lambda_{\gamma}^{0}$, say less than $6 \times 10^{-7} \mathrm{~cm}$, for the case of a Sodium D-line where $\lambda_{\gamma}^{0}=$ $5.9 \times 10^{-5} \mathrm{~cm}$.

The calculation of the refractive index is now repeated, first considering the case of a slab of transparent medium of thickness much greater than $\lambda_{\gamma}^{0}$, so that the approximation of the last member of (5.3) is no longer valid, but assuming that the value of $t_{D}-t_{0}$ is appreciably larger than the typical photon flight time between the source and the detector, and secondly, also considering a thick slab, but assuming that $t_{D}-t_{0}$ may take any value, including ones near to the minimum value fixed by the source-detector distance and the 


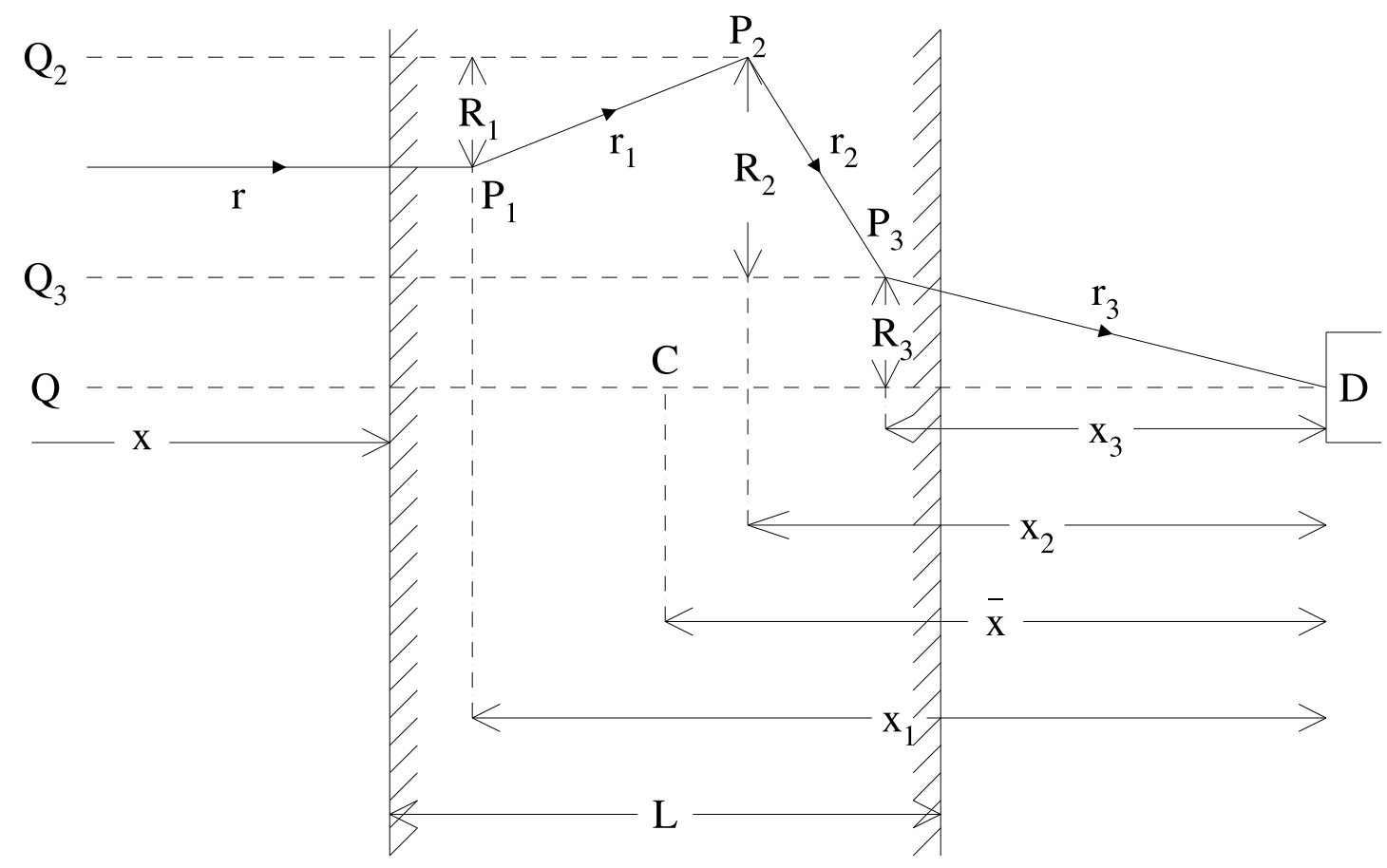

Figure 3: A photon produced by spontaneous decay of an atom at $S$, far to the left of the figure scatters at the points $P_{1}, P_{2}$ and $P_{3}$ inside a block of transparent material before being detected at $D$. The path $S_{1} P_{2} P_{3} D$ lies in the plane of the figure. Various geometrical parameters used in (5.13) are defined.

velocity of light in vacuum. In both cases $\rho=0$ is assumed, so that damping effects due to the finite lifetime of the source atom are neglected.

In order to calculate the refraction of light in a block of transparent material of thickness, $L$, much greater than $\lambda_{\gamma}^{0}$, it will be found necessary to sum over all possible configuations of multiple scattering of the photon from the atoms of the medium. In Fig.3 the source atom is situated far to the left, so that the path of the photon from the atom to the front surface of the block may be taken to be a constant, equal to the distance, $\mathrm{x}$, from the atom to the front face of the block. As in Fig.2, the photon detector, D, lies on the $\mathrm{x}$-axis, which is perpendicular to the faces of the block. The distance from the center of the block to the detector is $\bar{x}$. In Fig.3, the photon scatters at the points $P_{1}, P_{2}$ and $P_{3}$ which lie in a plane containing the x-axis, QD, and are at distances from $\mathrm{D}$, along this axis, of $x_{1}, x_{2}$ and $x_{3}$. The path lengths between the points $P_{1}$ and $P_{2}, P_{2}$ and $P_{3}$ and $P_{3}$ and $D$ are $r_{1}, r_{2}$ and $r_{3}$. Other possible paths of the photon in the block are generated by rotating the point $P_{1}$ about the axis $Q_{2} P_{2}$ to give the series of points: $P_{1}^{\prime}, P_{1}^{\prime \prime},, P_{1}^{\prime \prime \prime} \ldots$ all distant $r_{1}$ from $P_{2}$ and lying on a circle of radius $R_{1}$ in the yz plane. By varying the value of $R_{1}$ the integral is performed over the positions of all possible atomic scattering events between $x_{1}$ and $x_{1}+\Delta x_{1}$. Similarly, by considering the points $P_{2}^{\prime}, P_{2}^{\prime \prime},, P_{2}^{\prime \prime \prime} \ldots$ and $P_{3}^{\prime}, P_{3}^{\prime \prime},, P_{3}^{\prime \prime \prime} \ldots$ lying on circles of radius $R_{2}$ and $R_{3}$ respectively, centered on the axes $Q_{3} P_{3}$ and $Q D$, and integrating over all values of $R_{2}$ and $R_{3}$, all possible atomic scattering events in the intervals from $x_{2}$ to $x_{2}+\Delta x_{2}$ and $x_{3}$ to $x_{3}+\Delta x_{3}$ are summed. Including all paths with radii in the range $R_{i}$ to $R_{i}+\Delta R_{i},(\mathrm{i}=1,2,3)$ the single scattering formula analgous 
to (4.15), generalises, for the three-scattering case, to $^{20}$ :

$$
\begin{aligned}
\Delta A_{3} & =\frac{\tilde{\mathcal{A}}}{\left(x+\bar{x}+\frac{L}{2}-x_{1}\right)} \exp \left[-i \kappa\left(c\left(t_{D}-t_{0}\right)-\left(x+\bar{x}+\frac{L}{2}-x_{1}\right)\right)\right] \\
& \times \frac{\exp \left[i \kappa r_{1}\right]}{r_{1}} \mathcal{N} \mathcal{A}_{\text {scatt }} R_{1} \Delta R_{1} \Delta \phi_{1} \Delta x_{1} \\
& \times \frac{\exp \left[i \kappa r_{2}\right]}{r_{2}} \mathcal{N} \mathcal{A}_{\text {scatt }} R_{2} \Delta R_{2} \Delta \phi_{2} \Delta x_{2} \\
& \times \frac{\exp \left[i \kappa r_{3}\right]}{r_{3}} \mathcal{N} \mathcal{A}_{\text {scatt }} R_{3} \Delta R_{3} \Delta \phi_{3} \Delta x_{3}
\end{aligned}
$$

Changing the integration variables to $r_{1}, r_{2}$ and $r_{3}$, as in deriving (4.16) from (4.15) and performing the integrations, neglecting, as in (5.2), the contributions from the upper limits of the integrals, on performing the azimuthal integrations, due to their rapid phase variation, gives:

$$
\begin{aligned}
\Delta A_{3} & =\frac{\tilde{\mathcal{A}}}{\left(x+\bar{x}+\frac{L}{2}-x_{1}\right)} \exp \left[-i \kappa\left(c\left(t_{D}-t_{0}\right)-\left(x+\bar{x}+\frac{L}{2}\right)\right]\right. \\
& \times \exp \left[i \kappa\left(-x_{1}+\left(x_{1}-x_{2}\right)+\left(x_{2}-x_{3}\right)+x_{3}\right)\right]\left(\frac{i 2 \pi \mathcal{N} \mathcal{A}_{\text {scatt }}}{\kappa}\right)^{3} \Delta x_{1} \Delta x_{2} \Delta x_{3} \\
& \simeq \frac{\tilde{\mathcal{A}}}{x_{S D}} \exp \left[-i \kappa\left(c\left(t_{D}-t_{0}\right)-x_{S D}\right)\right]\left(\frac{i 2 \pi \mathcal{N} \mathcal{A}_{\text {scatt }}}{\kappa}\right)^{3} \Delta x_{1} \Delta x_{2} \Delta x_{3}
\end{aligned}
$$

Where the approximation $x+\bar{x}+\frac{L}{2}-x_{1} \simeq x+\bar{x}+\frac{L}{2}=x_{S D}$ has been made in the denominator, where $x_{S D}$ is the source-detector distance. Integrating now over $x_{1}, x_{2}$ and $x_{3}$ gives:

$$
\begin{aligned}
A(L)_{3} & =\frac{\tilde{\mathcal{A}}}{x_{S D}} \exp \left[-i \kappa\left(c\left(t_{D}-t_{0}\right)-x_{S D}\right)\right]\left(\frac{i 2 \pi \mathcal{N} \mathcal{A}_{\text {scatt }}}{\kappa}\right)^{3} \int_{x_{2}}^{\bar{x}+\frac{L}{2}} d x_{1} \int_{x_{3}}^{\bar{x}+\frac{L}{2}} d x_{2} \int_{\bar{x}-\frac{L}{2}}^{\bar{x}+\frac{L}{2}} d x_{3} \\
& =\frac{\tilde{\mathcal{A}}}{x_{S D}} \exp \left[-i \kappa\left(c\left(t_{D}-t_{0}\right)-x_{S D}\right)\right] \frac{1}{3 !}\left(\frac{i 2 \pi \mathcal{N} \mathcal{A}_{\text {scatt }} L}{\kappa}\right)^{3}
\end{aligned}
$$

The limits of the $x_{1}, x_{2}$ and $x_{3}$ integrals are determined by the inequalities (see Fig.2) $x_{1} \geq x_{2} \geq x_{3}$. In (5.15) the general expression for the nested $n$-fold integral:

$$
\mathcal{I}_{n}=\int_{x_{2}}^{\bar{x}+\frac{L}{2}} d x_{1} \int_{x_{3}}^{\bar{x}+\frac{L}{2}} d x_{2} \ldots \int_{\bar{x}-\frac{L}{2}}^{\bar{x}+\frac{L}{2}} d x_{n}=\frac{L^{n}}{n !}
$$

is used. This formula is derived in Appendix B. For the case of $n$ scatterings of the photon in the block, (5.15) generalises to:

$$
A(L)_{3}=\frac{\tilde{\mathcal{A}}}{x_{S D}} \exp \left[-i \kappa\left(c\left(t_{D}-t_{0}\right)-x_{S D}\right)\right] \frac{1}{n !}\left(\frac{i 2 \pi \mathcal{N} \mathcal{A}_{\text {scatt }} L}{\kappa}\right)^{n}
$$

\footnotetext{
${ }^{20}$ Note that in (5.13) only forward scattering processes are included. Back-scattering between two atoms separated by distances $\geq \lambda_{\gamma}^{0}$ results results in rapid variation of the phase of the path amplitude strongly suppressing such contributions. The vanishing of the amplitude for backward scattering in a uniform transparent medium can also be understood as an effect of destructive interference between different path amplitudes, as described in Section 7 below.
} 
Adding the contribution of the unscattered photon from (4.19) gives, for the fully integrated path amplitude (i.e. the probability amplitude) at the detector:

$$
\begin{aligned}
A(L)_{\text {tot }} & =A+A(L)_{1}+A(L)_{2}+\ldots \\
& =\frac{\tilde{\mathcal{A}}}{x_{S D}} \exp \left[-i \kappa\left(c\left(t_{D}-t_{0}\right)-x_{S D}\right)\right] \\
& \times\left[1+\frac{i 2 \pi \mathcal{N} \mathcal{A}_{\text {scatt }} L}{\kappa}+\frac{1}{2 !}\left(\frac{i 2 \pi \mathcal{N} \mathcal{A}_{\text {scatt }} L}{\kappa}\right)^{2}+\ldots\right] \\
& =\frac{\tilde{\mathcal{A}}}{x_{S D}} \exp \left[-i \kappa\left(c\left(t_{D}-t_{0}\right)-x_{S D}-\frac{2 \pi \mathcal{N} \mathcal{A}_{\text {scatt }} L}{\kappa^{2}}\right)\right] \\
& =\frac{\tilde{\mathcal{A}}}{x_{S D}} \exp \left[-i \kappa\left(c\left(t_{D}-t_{0}\right)-\left(x_{S D}-L+n L\right)\right)\right]
\end{aligned}
$$

Where the refractive index, $n$, defined in (5.8), has been introduced. Defining, as in (5.4) above, $f$ as the fraction of the total distance between the source atom and the photon detector occupied by the transparent medium:

$$
f=\frac{L}{x_{S D}}
$$

(5.18) may be written as:

$$
A(L)_{t o t}=\frac{\tilde{\mathcal{A}}}{x_{S D}} \exp \left[-i \kappa\left(c\left(t_{D}-t_{0}\right)-\frac{c x_{S D}}{v(f)}\right)\right]
$$

where:

$$
v(f)=\frac{c}{1+(n-1) f}
$$

which may be compared with (5.6) and (5.7) above. The phase of the amplitude in (5.20) agrees with that in (5.6) above, derived by considering single scattering of the photon in a thin sheet.

The calculation of the refractive index is now repeated taking properly into account the time interval $\Delta t \equiv t_{D}-t_{0}$ between the time of production of the excited atom $t_{0}$ and the time of photon detection $t_{D}$. Referring to Fig.3 it can be seen that, for the configuration of excited atom and detector shown there, the minimum possible value of $\Delta t$ is equal to the time of flight in vacuum, $x_{S D} / c$, of the photon between the excited atom and the detector and correponds to the straight line path SQCD between the source, S, and the detector. It also corresponds to the case when the excited atom decays promptly after production. For later detection times, the photon may follow paths displaced from the axis SQCD but, considering, for example, the case of triple scattering, the upper limits on the $r_{1}, r_{2}$ and $r_{3}$ integrals in (5.14) will now be restricted by the value of $\Delta s+x_{S D}$, which is the maximum path length allowed by the actual values of $t_{D}$ and $t_{0}$ and the source-detector distance $x_{S D}$ :

$$
\Delta s \equiv c\left(t_{D}-t_{0}\right)-x_{S D}=c \Delta t-x_{S D}
$$

Note that a path of length $\Delta s+x_{S D}$ still corresponds to prompt decay. Decays occuring at later times, for the same value of $t_{D}$, have path lengths less than $\Delta s+x_{S D}$. Inspection of Fig. 3 shows that $r_{1}, r_{2}$ and $r_{3}$ must satisfy the inequality:

$$
\Delta s \geq r_{1}+r_{2}+r_{3}-x_{1}
$$


Noting also that;

$$
\begin{aligned}
& r_{1} \geq x_{1}-x_{2} \\
& r_{2} \geq x_{2}-x_{3} \\
& r_{3} \geq x_{3}
\end{aligned}
$$

the following inequalities may be derived from (5.23):

$$
\begin{aligned}
x_{1}-x_{2} & \leq r_{1} \leq \Delta s-r_{2}-r_{3}+x_{1} \\
x_{2}-x_{3} & \leq r_{2} \leq \Delta s-r_{3}+x_{2} \\
x_{3} & \leq r_{3} \leq \Delta s+x_{3}
\end{aligned}
$$

In the present case, the definite integral derived from (5.13) above is:

$$
\begin{aligned}
A(L)_{3} & =\frac{\tilde{\mathcal{A}}}{x_{S D}} \exp \left[-i \kappa\left(c\left(t_{D}-t_{0}\right)-x_{S D}+x_{1}\right)\right]\left(2 \pi \mathcal{N} \mathcal{A}_{\text {scatt }}\right)^{3} \int_{x_{2}}^{\bar{x}+\frac{L}{2}} d x_{1} \int_{x_{3}}^{\bar{x}+\frac{L}{2}} d x_{2} \int_{\bar{x}-\frac{L}{2}}^{\bar{x}+\frac{L}{2}} d x_{3} \\
& \times \int_{x_{1}-x_{2}}^{\Delta s-r_{2}-r_{3}+x_{1}} \exp \left[i \kappa r_{1}\right] d r_{1} \int_{x_{2}-x_{3}}^{\Delta s-r_{3}+x_{2}} \exp \left[i \kappa r_{2}\right] d r_{2} \\
& \times \int_{x_{3}}^{\Delta s+x_{3}} \exp \left[i \kappa r_{3}\right] d r_{3}
\end{aligned}
$$

Performing the integrals over $r_{1}, r_{2}, r_{3}, x_{1}, x_{2}$ and $x_{3}$, as shown in Appendix C, yields the result:

$$
\begin{aligned}
A(L)_{3} & =\frac{\tilde{\mathcal{A}}}{x_{S D}} \exp \left[-i \kappa\left(c\left(t_{D}-t_{0}\right)-x_{S D}\right)\right] \\
& \times \frac{1}{3 !}\left(\frac{i 2 \pi \mathcal{N} \mathcal{A}_{\text {scatt }} L}{\kappa}\right)^{3}\left[\left(-\frac{(i \Delta \Phi)^{2}}{2 !}+i \Delta \Phi-1\right) \exp [i \Delta \Phi]+1\right]
\end{aligned}
$$

where:

$$
\Delta \Phi \equiv \kappa \Delta s
$$

The nested $r_{1}, r_{2}$ and $r_{3}$ integrals in (5.30) generalise in a straightforward manner to an arbitary number of photon scatterings. The expression for the probability amplitude in (5.20) above then replaced by:

$$
\begin{aligned}
A(L)_{t o t} & =\frac{\tilde{\mathcal{A}}}{x_{S D}} \exp \left[-i \kappa\left(c\left(t_{D}-t_{0}\right)-x_{S D}\right)\right] \\
& \times\left\{\exp [i \beta L]+\exp [i \Delta \Phi]\left[-i \beta L+\frac{(i \beta L)^{2}}{2 !}(i \Delta \Phi-1)\right.\right. \\
& +\frac{(i \beta L)^{3}}{3 !}\left(-\frac{(i \Delta \Phi)^{2}}{2 !}+i \Delta \Phi-1\right) \\
& \left.\left.+\frac{(i \beta L)^{4}}{4 !}\left(\frac{(i \Delta \Phi)^{3}}{3 !}-\frac{(i \Delta \Phi)^{2}}{2 !}+i \Delta \Phi-1\right)+\ldots\right]\right\}
\end{aligned}
$$

where

$$
\beta \equiv \frac{2 \pi \mathcal{N} \mathcal{A}_{\text {scatt }}}{\kappa}
$$

For vanishingly small values of $\Delta \Phi$, the term containing the large square brackets tends to the limiting value: $1-\exp [i \beta L]$ so that $A(L)_{\text {tot }}$ reduces to the vacuum path amplitude 
between the source and the detector and the refractive effect of the block of transparent medium disappears.

In order to discuss the probability amplitude for arbitary values of $\Delta \Phi$, it is convenient to re-write the infinite series in the curly brackets of (5.33) separated out into real and imaginary parts. The algebraic manipulations necessary for this are discussed in Appendix D. The following expression is obtained:

$$
\begin{aligned}
A(L)_{t o t} & =\frac{\tilde{\mathcal{A}}}{x_{S D}} \exp \left[-i \kappa\left(c\left(t_{D}-t_{0}\right)-x_{S D}\right)\right] \\
& \times\left\{1+\beta L[S+i(1-C)]+\frac{(\beta L)^{2}}{2 !}[[C-1+\Delta \Phi S+i[S-\Delta \Phi+\Delta \Phi(1-C)]]\right. \\
& +\frac{(\beta L)^{3}}{3 !}\left[\Delta \Phi-S+\Delta \Phi(C-1)+\frac{(\Delta \Phi)^{2}}{2 !} S\right. \\
& \left.+i\left[C-1+\frac{(\Delta \Phi)^{2}}{2 !}+\Delta \Phi(S-\Delta \Phi)+\frac{(\Delta \Phi)^{2}}{2 !}(1-C)\right]\right] \\
& +\frac{(\beta L)^{4}}{4 !}\left[1-\frac{(\Delta \Phi)^{2}}{2 !}-C+\Delta \Phi(\Delta \Phi-S)+\frac{(\Delta \Phi)^{2}}{2 !}(C-1)+\frac{(\Delta \Phi)^{3}}{3 !} S\right. \\
& \left.+i\left[\Delta \Phi-\frac{(\Delta \Phi)^{3}}{3 !}-S+\Delta \Phi\left(C-1+\frac{(\Delta \Phi)^{2}}{2 !}\right)-\frac{(\Delta \Phi)^{2}}{2 !}(\Delta \Phi-S)+\frac{(\Delta \Phi)^{3}}{3 !}(1-C)\right]\right] \\
& +\ldots\}
\end{aligned}
$$

where

$$
S \equiv \sin \Delta \Phi \quad, \quad C \equiv \cos \Delta \Phi
$$

This formula for the probability amplitude may be written in a more compact fashion by introducing a notation for truncated series expansions of trigonometric functions:

$$
\begin{aligned}
& S_{0}=0, \quad S_{1} \equiv \Delta \Phi, \quad S_{2} \equiv \Delta \Phi-\frac{(\Delta \Phi)^{3}}{3 !}, \quad S_{3} \equiv \Delta \Phi-\frac{(\Delta \Phi)^{3}}{3 !}+\frac{(\Delta \Phi)^{5}}{5 !}, \ldots \\
& C_{0}=1, \quad C_{1} \equiv 1-\frac{(\Delta \Phi)^{2}}{2 !}, \quad C_{2} \equiv 1-\frac{(\Delta \Phi)^{2}}{2 !}+\frac{(\Delta \Phi)^{4}}{4 !}, \quad, \ldots
\end{aligned}
$$

The expression in the large curly brackets of (5.35) is denoted as $F(\Delta \Phi, \beta L)_{\text {ref }}$. It is the complex amplitude that multiplies the vacuum probability amplitude $A$ to take into account the interaction of the photon with the atoms of the refractive medium. Using the definitions in (5.36) and (5.37), the real and imaginary parts of $F_{\text {ref }}$ may be written as:

$$
\begin{aligned}
R e F_{r e f}= & 1+\beta L S+\frac{(\beta L)^{2}}{2 !}\left(C+S S_{1}-1\right)+\frac{(\beta L)^{3}}{3 !}\left(C S_{1}-S C_{1}\right) \\
+ & \frac{(\beta L)^{4}}{4 !}\left(1-C C_{1}-S S_{2}\right)+\frac{(\beta L)^{5}}{5 !}\left(S C_{2}-C S_{2}\right)+\ldots \\
+ & (-1)^{n} \frac{(\beta L)^{2 n}}{2 n !}\left(1-C C_{n-1}-S S_{n}\right)+(-1)^{n} \frac{(\beta L)^{2 n+1}}{(2 n+1) !}\left(S C_{n}-C S_{n}\right) \\
& +\ldots
\end{aligned}
$$




$$
\begin{aligned}
I m F_{r e f}= & \beta L(1-C)+\frac{(\beta L)^{2}}{2 !}\left(S-C S_{1}\right)+\frac{(\beta L)^{3}}{3 !}\left(C C_{1}+S S_{1}-1\right) \\
+ & \frac{(\beta L)^{4}}{4 !}\left(C S_{2}-S C_{1}\right)+\frac{(\beta L)^{5}}{5 !}\left(1-C C_{2}-S S_{2}\right) \\
+ & (-1)^{n} \frac{(\beta L)^{2 n}}{2 n !}\left(C S_{n}-S C_{n-1}\right)+(-1)^{n} \frac{(\beta L)^{2 n+1}}{(2 n+1) !}\left(1-C C_{n}-S S_{n}\right) \\
& +\ldots
\end{aligned}
$$

(5.38) may also be written as:

$$
\begin{aligned}
R e F_{r e f}= & 1+\beta L S+\frac{\beta L}{2}(\beta L)\left(C+S S_{1}-1\right)+\frac{\beta L}{3} \frac{(\beta L)^{2}}{2 !}\left(C S_{1}-S C_{1}\right) \\
+ & \frac{\beta L}{4} \frac{(\beta L)^{3}}{3 !}\left(1-C C_{1}-S S_{2}\right)+\frac{\beta L}{5} \frac{(\beta L)^{4}}{4 !}\left(S C_{2}-C S_{2}\right) \\
& +\ldots \\
+ & \frac{\beta L}{2 n}\left[(-1)^{n} \frac{(\beta L)^{2 n-1}}{(2 n-1) !}\left(1-C C_{n-1}-S S_{n}\right)\right] \\
+ & \left.\frac{\beta L}{2 n+1}\left[(-1)^{n} \frac{(\beta L)^{2 n}}{(2 n) !}\left(S C_{n}-C S_{n}\right)\right)\right] \\
& +\ldots
\end{aligned}
$$

As will be discussed below, the values of quantities $\beta L$ and $\Delta \Phi$ typically satisfy the condition $\beta L \gg \Delta \Phi$. Inspection of successive terms in the series (5.39) and (5.40) shows that there is a one-to-one correspondence between terms with similar trigonometric coefficients (e.g. $1-C C_{1}-S S_{1}$ in (5.39) and $1-C C_{1}-S S_{2}$ in (5.40)). However, in (5.40), the $(\beta L)^{n} / n$ ! terms of (5.39) are multiplied by the numerically large factor $-\beta L / n$. The contribution of not-too-large values of $n$ to $R e F_{r e f}$ is then expected to be much larger than the corresponding contribution to $I m F_{\text {ref }}$. For large values of $n$ both series are expected to converge rapidly, since in this case, all the trigonometric factors tend to zero:

$$
\begin{gathered}
C C_{n}+S S_{n}-1 \simeq C^{2}+S^{2}-1=0 \\
S C_{n}-C S_{n} \simeq S C-C S=0
\end{gathered}
$$

It is then to be expected that, in general:

$$
\left|R e F_{r e f}\right| \gg\left|I m F_{r e f}\right|
$$

which implies that the phase, $\Phi_{F}$, of the amplitude $F_{\text {ref }}$ is close to zero, independently of the values of $\beta L$ and $\Delta \Phi$. In this case the phase of the vacuum path amplitude remains unchanged by the interaction of the photon with the atoms of the refractive medium and a phenomenon that may be called 'refraction annulment' is expected to occur. Refraction, i.e. propagation of light in the medium with an apparently reduced velocity $c / n$, only occurs when, as in (5.14), the contributions from the upper limits of the $r$ integrals vanish on integration over the azimuthal angles due to their rapid phase variation. The latter is induced by the geometric boundaries of the refractive medium that determine the phase of the integrand. These are effectively randomly placed at the distance scales of the order of the wavelength of light. This point is further discussed in Appendix A. However, for 


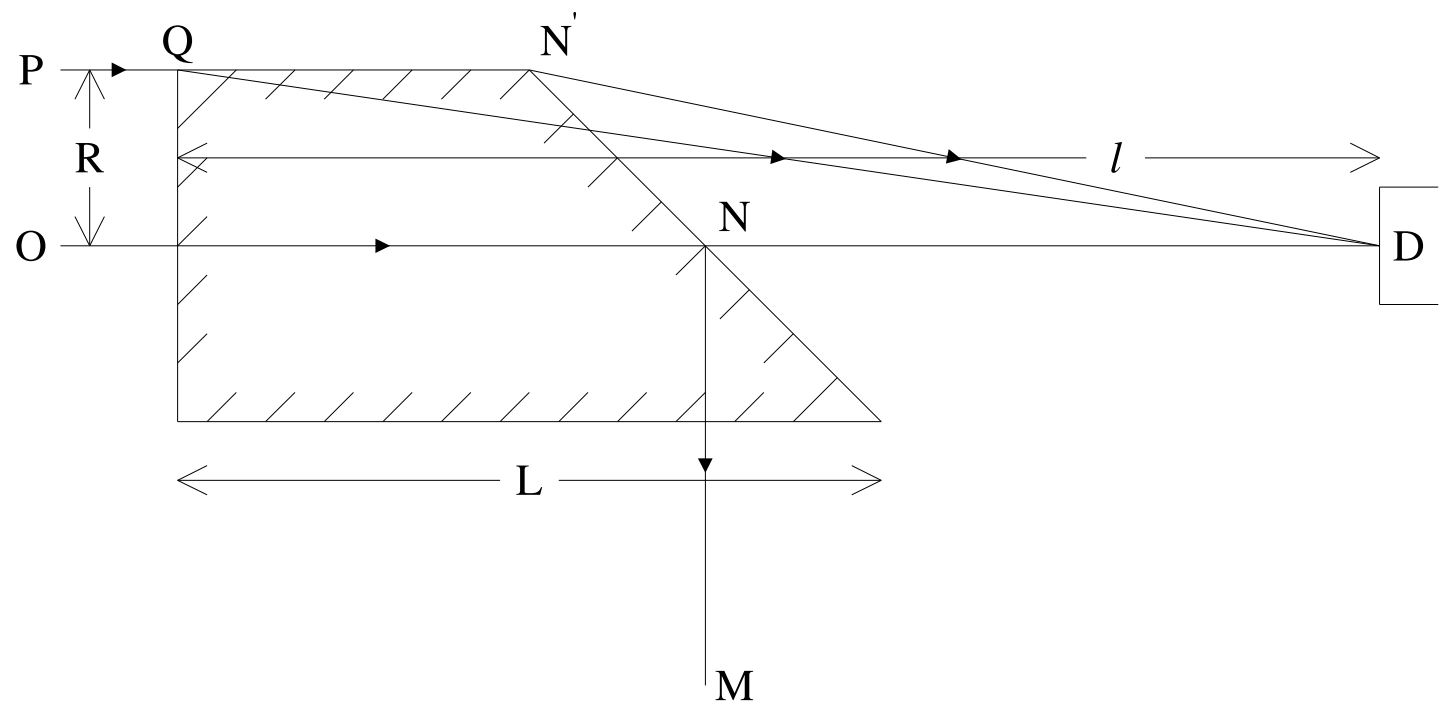

Figure 4: A simple experiment to search for refraction annulment. The side view is shown of a cylindrical glass block of refractive index $n=1.5$, radius $R$ and length $L$ with an oblique face containing the points $N$ and $N^{\prime}$. Far to the left is an intense photon source consisting of excited sodium atoms. Photons produced by spontaneous decay of the atoms corresponding to the minimum detection time $t_{D}$ for a given production time $t_{0}$ of the excited atom, can reach the detector $D$ only by paths near $O N$ and for decays occuring promptly after production of the excited atom. In this case the phase change of the probability amplitude due to interactions with the atoms of the block is strongly suppressed, the effective refractive index is very low, and so the photon can be transmitted through the oblique face and detected at D. For later detection times, photons may be produced at later decay times, and all photon paths between $O N$ and $P Q N$ ' are available. Normal refraction then occurs in the glass, and each photon is totally internally reflected at the oblique face (e.g. the path ONM). 
small values of $\Delta s$, the photon paths always remain away from the transverse boundaries of the refractive material so that the latter have no effect on the value of the integral over paths. This is the case in (5.33).

To estimate the importance of the refraction annulment effect consider the simple experimental configuration sketched in Fig.4. A prism is constructed by cutting obliquely the face of a glass cylinder of length, $L$, and radius, $R$, at $45^{\circ}$ to the axis of the cylinder. In Fig.4, the normal to the oblique plane face of the prism is in the plane of the diagram. The source atom is assumed to be at a large distance to the left along the $\mathrm{x}$-axis, which coincides with that of the cylinder, so that all photons from the source have essentially the same time-of-flight to the front face of the cylinder. The photon detector, $D$, is situated on the x-axis at distance $l$ from the front face of the prism. The largest possible value of $\Delta s$ for all the $r$ integrals to be limited by the production time of the excited atom, rather than by the geometrical boundaries of the prism, corresponds to a photon trajectory such as PQD. A simple geometrical calculation yields:

$$
\Delta \Phi^{\max }=\frac{2 \pi}{\lambda_{\gamma}^{0}} \Delta s^{\max }=\frac{2 \pi}{\lambda_{\gamma}^{0}} \frac{R^{2}}{2 l}+O\left(\frac{R^{4}}{\lambda_{\gamma}^{0} l^{3}}\right)
$$

If the exited atom is sodium in the upper state of the D-line transition, $\lambda_{\gamma}^{0}=\lambda_{D}=$ $5.9 \times 10^{-5} \mathrm{~cm}$. With $R=5 \mathrm{~cm}$ and $l=200 \mathrm{~cm}, \Delta s^{\max }=625 \mu \mathrm{m}$ and $\Delta \Phi^{\max }=6.66 \times 10^{3}$ rad or 1060 periods. Assuming a refractive index for the sodium D-line of $n=1.5$ and using (5.9) and (5.34) with $L=40 \mathrm{~cm}$ gives

$$
\beta L=\frac{2 \pi(n-1) L}{\lambda_{D}}=\frac{\pi L}{\lambda_{D}}=2.12 \times 10^{6}
$$

Thus, in this case, $\beta L \gg \Delta \Phi^{\max } \gg 1$, so that, according to the argument given above, the phase of $F_{r e f}$, and hence the corresponding refractive index, is expected to be very small. For a given geometrical configuration, the ratio of $\beta L$ to $\Delta \Phi^{\max }$ is a constant, independent of the dimensions of the apparatus. In order for the upper limits of the $r$ integrals to be independent of the geometrical boundaries of the prism, the excited atom must decay earlier than a time $\Delta s^{\max } / c=2.2 \times 10^{-12} \mathrm{sec}$ after production. Since the lifetime of the excited state producing the sodium D-lines is $5.4 \times 10^{-8}$ sec, a fraction $\simeq 4 \times 10^{-4}$ of all D-line photons emitted into the solid angle of the detector is then expected to cross the prism following an unrefracted trajectory close to OND, as if they were propagating in free space. These are the atoms that decay during the time interval: $0<t<\Delta s^{\max } / c$ after production. A simple experiment using photographic or photon counting techniques would be sufficient to confirm of invalidate this prediction. For larger values of $\Delta s$ (i.e. larger values of $t_{D}-t_{0}$ ) such that the upper limits of the $r$ integrals are determined by the geometrical boundaries of the prism, as in (5.14), the usual refraction phenomenon will occur in the medium and all the photons from the source will be internally reflected from the oblique face of the prism $^{21}$ so that the photons will follow trajectories close to ONM in Fig.4.

\footnotetext{
${ }^{21}$ The critical angle of incidence for total internal reflection is $42^{\circ}$ for $n=1.5$.
} 


\section{The Laws of Reflection, Refraction and Rectilinear Propagation of Light, and Fermat's Principle}

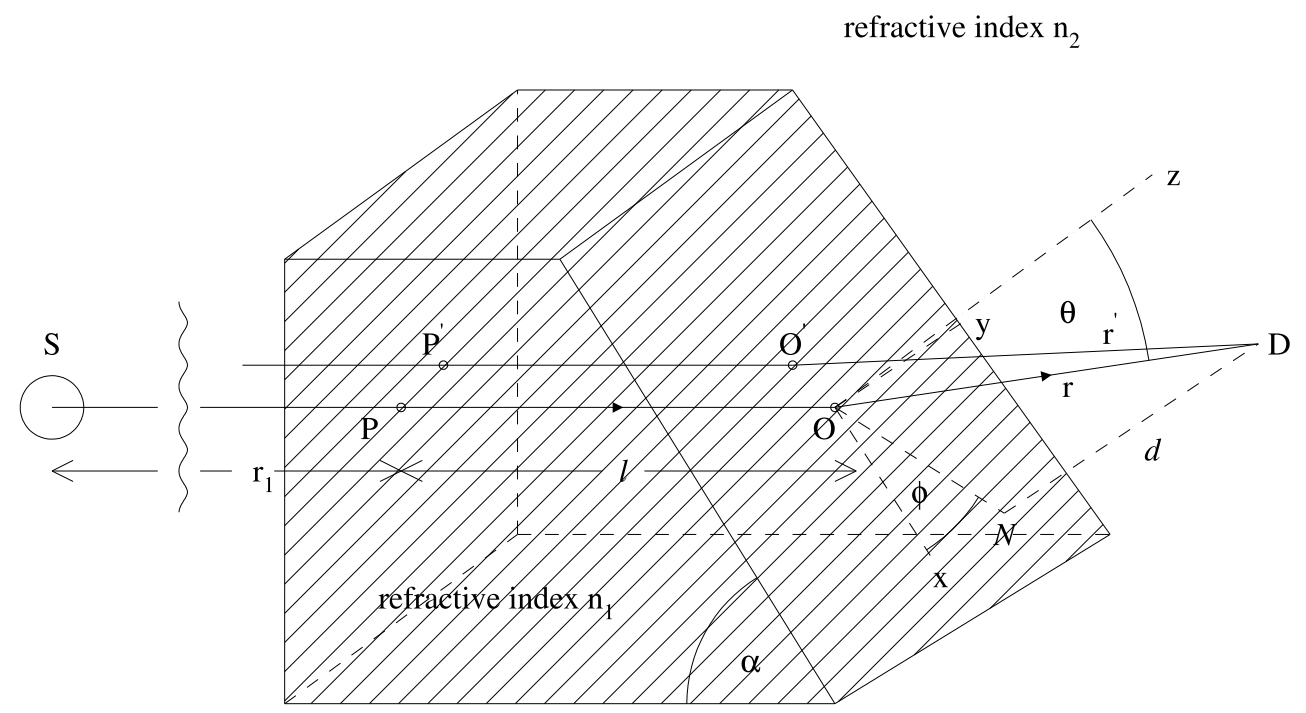

Figure 5: A photon produced by the source $S$ is observed in a detector at $D$, which is movable in a plane distant $d$ from the oblique face of a block of transparent material of refractive index $n_{1}$ surrounded by a medium of refractive index $n_{2}$. The position of $D$ that corresponds to an extremum of the phase of the probability amplitude for photon detection at $D$ is found to correspond to Snell's law of refraction.

In Fig. 5 is shown the trajectory, SPOD, of a photon from the decay of an excited atom, $\mathrm{S}$, at a point sufficiently far to the left of the figure that all straight line paths from the atom may be considered parallel to the line PO. Over the path segment, PO, of length, $l$, the photon propagates in a transparent medium of refractive index $n_{1}$. The surrounding space, back to the position of the excited atom, is assumed to be filled with a transparent medium of refractive index $n_{2}$. The interface between the two media, passing through $\mathrm{O}$, is a plane surface whose normal is at an angle $\theta_{I}=\pi / 2-\alpha$ to the segment PO (see Fig. $6 \mathrm{a})$, where, in the plane defined by PO and the normal, the angle between the projection of the interface and PO is $\alpha$. The photon detector, $\mathrm{D}$, is at a fixed distance, $d$, from the plane interface at a position defined by spherical polar coordinates $r, \theta, \phi$. The cartesian coordinate axes are defined so that the $z$ axis lies along the normal to the interface and the path segment $\mathrm{PO}$ is in the $x z$ plane.

By analogy with (5.18) the probability amplitude corresponding to detection of the photon at $\mathrm{D}$ at time $t_{D}$ is ${ }^{22}$ :

$$
\mathcal{A}=\frac{\tilde{\mathcal{A}}}{r_{1} l r} \exp \left[-\kappa c\left(t_{D}-t_{0}-\frac{n_{2} r_{1}}{c}\right)+i \Phi(r, l)\right]
$$

where

$$
\Phi(r, l) \equiv \kappa\left(n_{2} r+n_{1} l\right)
$$

\footnotetext{
${ }^{22}$ In this section it is assumed that $c\left(t_{D}-t_{0}\right)-x_{S D}$ is sufficiently large that there is no refraction annulment effect.
} 


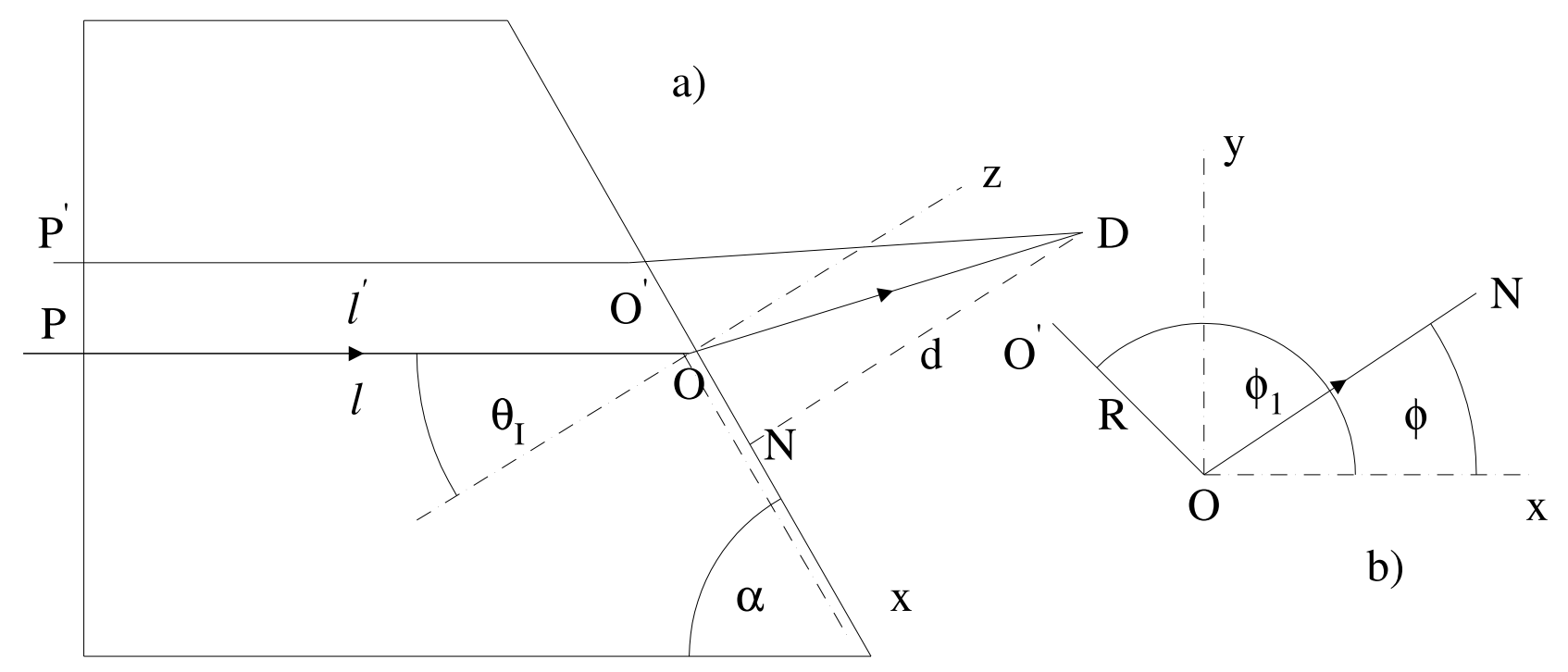

Figure 6: a) $x z$ projection, b) $x y$ projection, of the experimental layout of Fig.5.

The position of the detector $\mathrm{D}$ that maximises the photon detection probability is now determined by requiring that $\Phi(r, l)$ is stationary. As first pointed out by Dirac [20], and much emphasised later by Feynman [6], this condition ensures that neighbouring trajectories have almost the same phase, giving an amplitude, by vector addition in the complex plane, that maximises the modulus of the probability amplitude $A_{f i}$ in (2.1) above. The phase is varied by parallel displacement of the trajectory to P'O', where the point $\mathrm{O}^{\prime}$, in the $x y$ plane is specified by the coordinates: $x=R \cos \phi_{1}, y=R \sin \phi_{1}$ so that (see Figs.5 and 6) $r \rightarrow r^{\prime}$ and $l \rightarrow l^{\prime}$ where

$$
\begin{aligned}
r^{\prime 2} & =\left(d \tan \theta \cos \phi-R \cos \phi_{1}\right)^{2}+\left(d \tan \theta \sin \phi-R \sin \phi_{1}\right)^{2}+d^{2} \\
l^{\prime} & =l+R \cos \phi_{1} \cos \alpha
\end{aligned}
$$

The parameters $\theta, \phi, R$ and $\phi_{1}$ are now varied so that the $r, l$ dependent part, $\Phi$, of the phase in (6.1) is stationary. This requires that:

$$
n_{2} \frac{\partial r^{\prime}}{\partial R}+n_{1} \frac{\partial l^{\prime}}{\partial R}=n_{2} \frac{\partial r^{\prime}}{\partial \phi_{1}}+n_{1} \frac{\partial l^{\prime}}{\partial \phi_{1}}=0
$$

Differentiating (6.2) and (6.3):

$$
\begin{aligned}
\frac{\partial r^{\prime}}{\partial R} & =-\frac{d \tan \theta}{r^{\prime}}\left(\cos \phi \cos \phi_{1}+\sin \phi \sin \phi_{1}\right)+\frac{R}{r^{\prime}} \\
\frac{\partial r^{\prime}}{\partial \phi_{1}} & =\frac{R d \tan \theta}{r^{\prime}}\left(\cos \phi \sin \phi_{1}-\sin \phi \cos \phi_{1}\right) \\
\frac{\partial l^{\prime}}{\partial R} & =\cos \phi_{1} \cos \alpha \\
\frac{\partial l^{\prime}}{\partial \phi_{1}} & =-R \sin \phi_{1} \cos \alpha
\end{aligned}
$$

Substituting (6.5) and (6.7) into the first member of (6.4) gives:

$$
-\frac{n_{2} d \tan \theta\left(\cos \phi \cos \phi_{1}+\sin \phi \sin \phi_{1}\right)}{r^{\prime}}+n_{2} \frac{R}{r^{\prime}}+n_{1} \cos \phi_{1} \cos \alpha=0
$$


The solution to this equation with $R=\phi=0$ is:

$$
\left(-\frac{n_{2} d \tan \theta}{r}+n_{1} \cos \alpha\right) \cos \phi_{1}=0
$$

Since $d / r=\cos \theta,(6.10)$ gives:

$$
\sin \theta \equiv \sin \theta_{O}=\frac{n_{1}}{n_{2}} \cos \alpha=\frac{n_{1}}{n_{2}} \sin \theta_{I}
$$

which is Snell's law of refraction. Substituting (6.6) and (6.8) into the second member of (6.4) and setting $\phi=0$ gives:

$$
R\left(\frac{n_{2} d \tan \theta}{r}-n_{1} \cos \alpha\right) \sin \phi_{1}=0
$$

which is verified both by the condition $R=0$ and (6.11). (6.10) and (6.12) show that the phase is stationary for an arbitary value of $\phi_{1}$ provided that $R=\phi=0$.

When $\alpha=\pi / 2$, so that the photon is incident normally on the plane interface, so that $\theta_{I}=0,(6.11)$ gives $\theta_{O}=0$. The stationary phase condition then requires rectilinear propagation of the photon between $\mathrm{S}$ and $\mathrm{D}$.

The above calculation is easily adapted to the case of reflection at the interface, within the medium of refractive index $n_{1}$, by the replacements in (6.12):

$$
\theta \rightarrow \pi-\theta_{R}, \quad n_{2} \rightarrow n_{1}
$$

where $\theta_{R}$ denotes the angle of reflection relative to the inward normal at the interface. In this case (6.10) gives:

$$
n_{1}\left(-\sin \theta_{R}+\sin \theta_{I}\right) \cos \phi_{1}=0
$$

i.e., $\theta_{R}=\theta_{I}$, the law of reflection.

The solution, (6.11), for the stationary phase with $R=\phi=0$ and with $\theta$ determined by Snell's law, implies that the photon trajectory corresponding to the stationary phase of the path amplitude lies in the $x z$ plane defined by the incident trajectory $\mathrm{PO}$ and the normal to the surface separating the refractive media.

The sizes of the deviations to be expected from the classical trajectory corresponding to the stationary phase condition, as well as the spatial extent, within the media, of the regions where photon scattering processes contribute significantly to refraction or reflection will now be estimated using the path amplitude formalism.

In the region of the stationary point, the variation of the phase is determined by the second order partial derivatives of the phase with respect to the trajectory parameters. For the angle of refraction $\theta_{O}$, Taylor's theorem gives:

$$
\Delta \Phi=\Phi-\Phi_{\text {stat }}=\frac{1}{2}\left[\frac{\partial^{2} \Phi}{\partial R^{2}}\left(\frac{d R}{d \theta}\right)^{2}+\frac{\partial^{2} \Phi}{\partial \phi_{1}^{2}}\left(\frac{d \phi_{1}}{d \theta}\right)^{2}\right]_{\text {stat }}\left(\Delta \theta_{O}\right)^{2}
$$


From (6.1):

$$
\begin{aligned}
& \frac{\partial^{2} \Phi}{\partial R^{2}}=\kappa\left[n_{2} \frac{\partial^{2} r^{\prime}}{\partial R^{2}}+n_{1} \frac{\partial^{2} l^{\prime}}{\partial R^{2}}\right] \\
& \frac{\partial^{2} \Phi}{\partial \phi_{1}^{2}}=\kappa\left[n_{2} \frac{\partial^{2} r^{\prime}}{\partial \phi_{1}^{2}}+n_{1} \frac{\partial^{2} l^{\prime}}{\partial \phi_{1}^{2}}\right]
\end{aligned}
$$

Taking the $\mathrm{R}$ derivative of (6.5):

$$
\frac{\partial^{2} r^{\prime}}{\partial R^{2}}=\frac{1}{r^{\prime 2}} \frac{\partial r^{\prime}}{\partial R} d \tan \theta\left(\cos \phi \cos \phi_{1}+\sin \phi \sin \phi_{1}\right)+\frac{1}{r^{\prime}}-\frac{R}{r^{\prime 2}} \frac{\partial r^{\prime}}{\partial R}
$$

Choosing now $\phi_{1}=0$ so that the photon trajectory is in the $x z$ plane, and substituting the parameters at the stationary point: $R=\phi=0$ in Eqns (6.5) and (6.17) gives:

$$
\begin{aligned}
\left.\frac{\partial r^{\prime}}{\partial R}\right|_{\text {stat }} & =-\frac{d \tan \theta_{O}}{r} \\
\left.\frac{\partial^{2} r^{\prime}}{\partial R^{2}}\right|_{\text {stat }} & =\left.\frac{d}{r} \frac{\partial r^{\prime}}{\partial R}\right|_{\text {stat }} \tan \theta_{O}+\frac{1}{r}
\end{aligned}
$$

Combining (6.18) and (6.19):

$$
\begin{aligned}
\left.\frac{\partial^{2} r^{\prime}}{\partial R^{2}}\right|_{\text {stat }} & =-\frac{d^{2}}{r^{3}} \tan ^{2} \theta_{O}+\frac{1}{r} \\
& =\frac{1}{r}\left[1-\left(\frac{d}{r}\right)^{2} \tan ^{2} \theta_{O}\right] \\
& =\frac{\cos ^{2} \theta_{O}}{r}
\end{aligned}
$$

The $\mathrm{R}$ derivative of (6.7) gives:

$$
\frac{\partial^{2} l^{\prime}}{\partial R^{2}}=0
$$

(6.15), (6.20) and (6.21) may be combined to obtain:

$$
\left.\frac{\partial^{2} \phi}{\partial R^{2}}\right|_{s t a t}=\kappa n_{2} \frac{\cos ^{2} \theta_{O}}{r}
$$

Since $\cos \theta=d / r$,

$$
\left.\frac{d \cos \theta}{d R}\right|_{\text {stat }}=-\left.\sin \theta_{O} \frac{d \theta}{d R}\right|_{\text {stat }}=-\left.\frac{d}{r^{2}} \frac{d r}{d R}\right|_{\text {stat }}=-\left.\frac{d}{r^{2}} \frac{\partial r^{\prime}}{\partial R}\right|_{\text {stat }}=\frac{\sin \theta_{O} \cos \theta_{O}}{r}
$$

That is,

$$
\left.\frac{d R}{d \theta}\right|_{\text {stat }}=-\frac{r}{\cos \theta_{O}}
$$

Combining (6.14), (6.22) and (6.24) and noting that, since $\phi_{1}$ is chosen to be zero, the second term in square bracket in (6.14) vanishes,

$$
\Delta \Phi=\left.\frac{1}{2} \frac{\partial^{2} \Phi}{\partial R^{2}}\right|_{\text {stat }}\left(\left.\frac{d R}{d \theta}\right|_{\text {stat }}\right)^{2}\left(\Delta \theta_{O}\right)^{2}=\frac{\kappa n_{2} r}{2}\left(\Delta \theta_{O}\right)^{2}
$$


With $\Delta \Phi=\pi$, the spread of the angle $\theta_{O}$ about its classical value is then estimated to be:

$$
\Delta \theta_{O}=\sqrt{\frac{2 \pi}{\kappa n_{2} r}}=\sqrt{\frac{\lambda_{\gamma}^{0}}{n_{2} r}}
$$

In a similar way, the displacements parallel to the $x$ and $y$ axes, $\Delta x_{0}$ and $\Delta y_{0}$, corresponding to $\Delta \Phi=\pi$ are found to be:

$$
\begin{aligned}
\Delta y_{O} & =\sqrt{\frac{\lambda_{\gamma}^{0} r}{n_{2}}} \sec \theta_{O} \\
\Delta x_{O} & =\sqrt{\frac{\lambda_{\gamma}^{0} r}{n_{2}}}
\end{aligned}
$$

So, for the Sodium D-lines with $\lambda_{\gamma}^{0}=5.9 \times 10^{-5} \mathrm{~cm}$, refractive index of 1.5 and $r=1 \mathrm{~m}$, and normal incidence, $\Delta x_{O}=\Delta y_{0}=6.3 \times 10^{-4} \mathrm{~cm}$. If the transverse dimensions of the interface are larger than this the photon paths are well represented by the classical lightray corresponding to the stationary phase condition. For significantly smaller dimensions this is no longer the case and the photon paths are smeared over a much wider region. This is the diffraction-dominated domain of the classical wave theory of light.

Introducing the effective velocity, $v$, of the photon in the refractive medium: $v=c / n$ according to (5.6) the phase $\Phi(r, l)$ defined after (6.1) may be written as:

$$
\Phi(r, l)=\kappa\left(n_{2} v_{2} t_{2}^{e f f}+n_{1} v_{1} t_{1}^{e f f}\right)=\kappa c\left(t_{2}^{e f f}+t_{1}^{e f f}\right)=\kappa c T^{e f f}
$$

where

$$
t_{1}^{e f f}=\frac{l}{v_{1}}, \quad t_{2}^{e f f}=\frac{r}{v_{2}}
$$

and $T^{e f f}=t_{2}^{e f f}+t_{1}^{e f f}$ is the total effective propagation time of a photon from $\mathrm{P}$ to $\mathrm{D}$ in Fig.5. Since the propagation time from $\mathrm{S}$ to $\mathrm{P}$ ' is a constant, independant of the position of $\mathrm{P}$ ', a stationary value of $\Phi$ correponds also to a stationary value of the total effective propagation time from the source to $\mathrm{D}$. Thus the classical photon trajectory passing through D correponds to a stationary value of the photon's total effective propagation time, which is Fermat's Principle.

\section{The Reflection Coefficient of Light at Normal Inci- dence from a Plane Interface between Transparent Media}

A practical experimental set-up to measure the reflection coefficient of light is shown in Fig 7. A plane interface between vacuum and a medium of refractive index $n$ lies in the $y z$ plane of a Cartesian coordinate system. $S$ is a light source (as previously a single excited atom produced at time $\left.t_{0}\right)$ at a large distance $x=-x_{0}\left(x_{0}>0\right)$ from the interface. In an actual experiment this could conveniently be replaced by a small source in the focal 


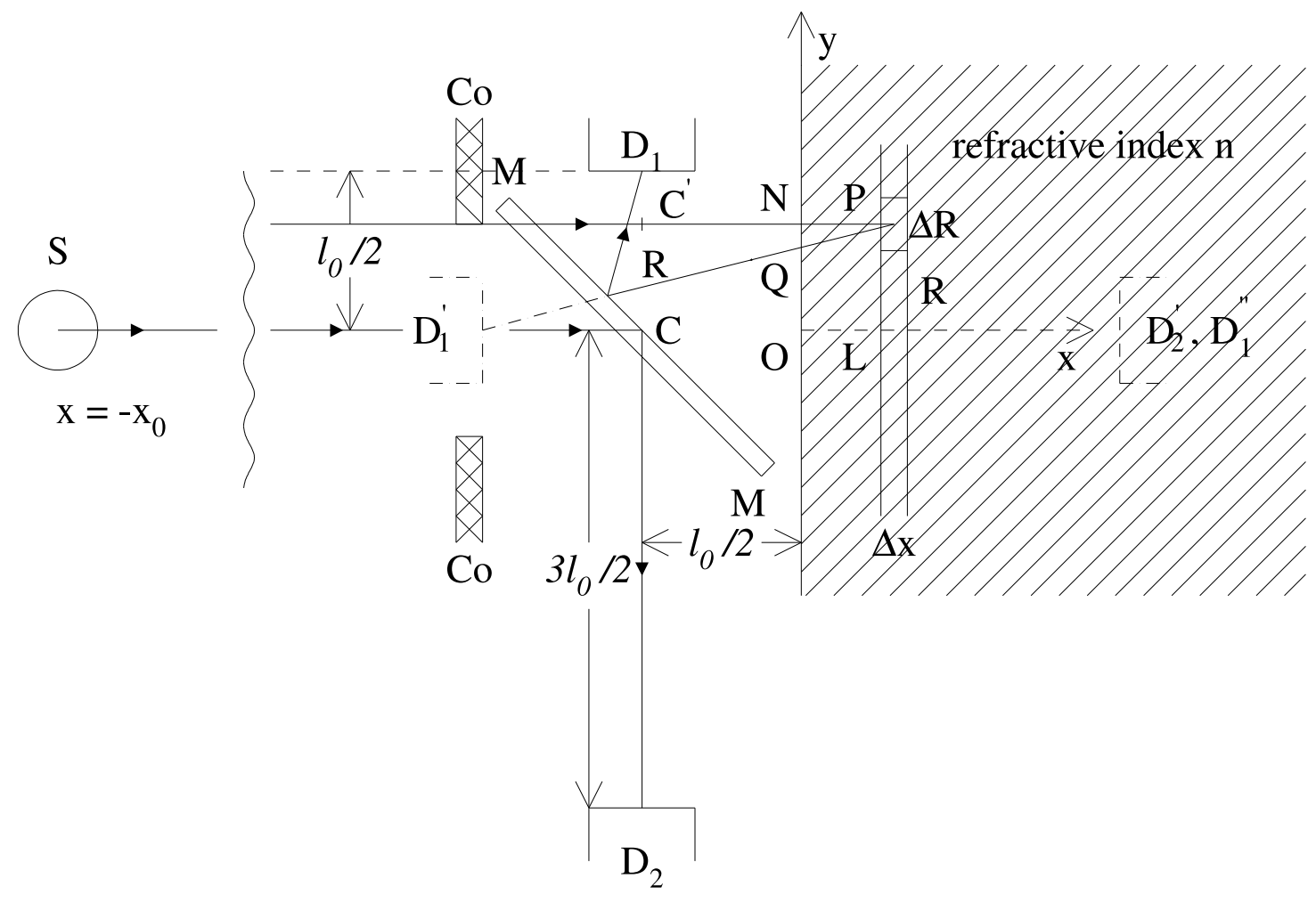

Figure 7: An apparatus to measure the reflection coefficient at normal incidence from a plane interface between vacuum and a block of uniform transparent material of refractive index $n$ (see text).

plane of a converging lens or by a parallel laser beam. At $(x, y, z)=\left(-l_{0} / 2,0,0\right)$ is a half-silvered mirror (HSM) (or an equivalent optical component) MM, with the normal to its surface in the $x y$ plane at an angle of $45^{\circ}$ to the $x$-axis. A circular collimator CoCo restricts the photon paths to a cylindrical region around the $x$-axis. Two small photon detectors $D_{1}$ and $D_{2}$ are situated at $\left(-l_{0} / 2, l_{0} / 2,0\right)$ and $\left(-l_{0} / 2,-3 l_{0} / 2,0\right)$ respectively. The virtual images of $D_{1}$ and $D_{2}$ generated by reflection in the HSM, as viewed from $\mathrm{O}$ and $\mathrm{S}$ respectively, are denoted as $D_{1}^{\prime}$ and $D_{2}^{\prime}$, and that generated by reflection of $D_{1}$ in both the HSM and the surface of the transparent medium, as viewed from $\mathrm{S}$, by $D_{1}^{\prime \prime}$. Since $D_{2}^{\prime}$ and $D_{1}^{\prime \prime}$ coincide (see Fig 7 ) it can be seen that, with this arrangement, equal solid angles are subtended at the detectors by the source $S$. Photons detected in $D_{1}$ cross the HSM, are backscattered from the transparent medium and reflected at the back surface of the HSM (e.g. path SNPQR $D_{1}$ ). Photons detected in $D_{2}$ are reflected from the HSM directly into $D_{2}$ (e.g. path $\mathrm{SC} D_{2}$ ).

It will be assumed that the decay width of the excited atom may be neglected (i.e. $\rho=0$ in (4.11)). The time dependence of the path amplitude for a given detection time $t_{D}$ is then $\simeq \exp \left[-i \kappa c\left(t_{D}-t_{0}\right)\right]$. Assuming that the back surface of the HSM is halfsilvered, as typically done in Michelson interferometers, which have a similar geometry, the probability amplitude for the photon to be observed in $D_{2}$ is ${ }^{23}$ :

$$
\mathcal{A}\left(D_{2}\right)=\mathcal{A}_{0} \mathcal{A}_{D} T_{H S M}^{2} R_{H S M} \exp \left[i\left(2 \phi_{H S M}^{T}+\phi_{H S M}^{R}\right)\right] \exp \left[i \frac{3 \kappa l_{0}}{2}\right]
$$

\footnotetext{
${ }^{23}$ Note that a photon detected in $D_{2}$ crosses the HSM twice
} 
where $\mathcal{A}_{0} \mathcal{A}_{D}$ denotes the probability amplitude for photon detection if $D_{2}$ were situated in the plane $x_{0}=-l_{0} / 2$, in the absence of the HSM, (including also the time dependent factor mentioned above), and $\mathcal{A}_{D}$ is the process amplitude for photon detection. $T_{H S M} \exp \left[i \phi_{H S M}^{T}\right]$ and $R_{H S M} \exp \left[i \phi_{H S M}^{R}\right]$ are the transmisson and reflection amplitudes of the HSM.

The path amplitude, $\Delta \mathcal{A}\left(D_{1}\right)$ for the photon to be scattered at some point, $\mathrm{P}$, in the interior of the transparent medium, and subsequently be detected in $D_{1}$ is given by the product of seven process amplitudes:

$$
\begin{aligned}
\Delta \mathcal{A}\left(D_{1}\right)= & \mathcal{A}_{D}\langle D|\gamma| R\rangle R_{H S M} \exp \left[i \phi_{H S M}^{R}\right]\langle R|\gamma| P\rangle \\
& \times\left(\mathcal{N} \mathcal{A}_{\text {scat }} R \Delta R \Delta x \Delta \phi\right)\left\langle P|\gamma| C^{\prime}\right\rangle T_{H S M} \exp \left[i \phi_{H S M}^{T}\right] \mathcal{A}_{0}
\end{aligned}
$$

Here $\mathrm{C}^{\prime}$ is the intersection with the plane $x=-l_{0} / 2$ of the photon path $\mathrm{SN}$, and $(R, \phi, x)$ are cylindrical coordinates specifying the position of P. Using (5.18) to write explicitly the triple product of photon propagators in (7.2), and integrating over all possible positions, $\mathrm{P}$, of the scattering process gives

$$
\mathcal{A}\left(D_{2}\right)=\iiint \tilde{\mathcal{A}}_{1} \exp \left[i \kappa\left[\frac{l_{0}}{2}+l-r+n(x+r)\right]\right] \frac{\mathcal{N} \mathcal{A}_{\text {scat }} R d R d x d \phi}{l}
$$

where

$$
\tilde{\mathcal{A}}_{1} \equiv \mathcal{A}_{0} \mathcal{A}_{D} R_{H S M} \exp \left[i \phi_{H S M}^{R}\right] T_{H S M} \exp \left[i \phi_{H S M}^{T}\right]
$$

In (7.3) the following spatial intervals have been introduced (see Fig 7):

$$
l \equiv P D_{1}^{\prime}, \quad r \equiv P Q, \quad x \equiv N P=O L
$$

Noting the relation:

$$
\frac{l_{0}}{x}=\frac{l-r}{r}
$$

which is a consequence of the similarity of the triangles $D_{1}^{\prime} Q O$ and $D_{1}^{\prime} P L$, the distance $r$ may be eliminated from (7.3) to give:

$$
\mathcal{A}\left(D_{2}\right)=\iiint \tilde{\mathcal{A}}_{1} \exp \left[i \kappa\left[\frac{l\left(l_{0}+n x\right)}{l_{0}+x}+\frac{l_{0}}{2}+n x\right]\right] \frac{\mathcal{N} \mathcal{A}_{\text {scat }} R d R d x d \phi}{l}
$$

As, according to the Huygens-Fresnel Principle, only values of $x$ of order $\lambda_{\gamma}^{0}$ contribute to the $x$ integral in (7.3), it follows that, in the integrand of (7.3), $x, r \ll l_{0}$ so that $l^{2}=R^{2}+\left(l_{0}+x\right)^{2} \simeq R^{2}+l_{0}^{2}$ is a good approximation. Thus $R d R=l d l$. Using this relation to eliminate $R$ in favour of $l$ in (7.5) and performing the $l$ integration, in a similar way to the $r_{1}$ integration in (5.1), gives:

$$
\begin{aligned}
\mathcal{A}\left(D_{2}\right)= & \iint \frac{\tilde{\mathcal{A}}_{1}\left(l_{0}+x\right)}{i \kappa\left(l_{0}-n x\right)} \mathcal{N} \mathcal{A}_{\text {scat }} d x d \phi \\
& \times\left\{\exp \left[i \kappa\left[\frac{l_{\text {max }}(\phi)\left(l_{0}+n x\right)}{l_{0}+x}+\frac{l_{0}}{2}-n x\right]\right]-\exp \left[i \kappa\left[\frac{3 l_{0}}{2}+2 n x\right]\right]\right\}
\end{aligned}
$$

The lower limit of the integral is $l_{\min }=l_{0}+x$. On performing the $\phi$ integration the contribution from the upper limit of the $l$ integral vanishes due to the rapid phase variation 
resulting from irregularities of size $\lambda_{\gamma}^{0}$ or larger in the shape of the collimator Co that determines $l_{\max }(\phi)$.

Performing the $x$ integral according to the Huygens-Fresnel Principle (the integral is equal to one half of the contribution of the first half-period in $x$ ), and neglecting $x$ relative to $l_{0}$, except in the phases of the path amplitudes, gives the final result for $\mathcal{A}\left(D_{2}\right)$ :

$$
\begin{aligned}
\mathcal{A}\left(D_{2}\right) & =-\tilde{\mathcal{A}}_{1}\left(\frac{\pi \mathcal{N} \mathcal{A}_{\text {scat }}}{n \kappa^{2}}\right) \exp \left[i \frac{3 \kappa l_{0}}{2}\right] \\
& =-\frac{\tilde{\mathcal{A}}_{1}}{2 n}\left(\frac{1}{2 \pi}\left(\lambda_{\gamma}^{0}\right)^{2} \mathcal{N} \mathcal{A}_{\text {scat }}\right) \exp \left[i \frac{3 \kappa l_{0}}{2}\right] \\
& =-\tilde{\mathcal{A}}_{1}\left(\frac{n-1}{2 n}\right) \exp \left[i \frac{3 \kappa l_{0}}{2}\right]
\end{aligned}
$$

where, in the last line, (5.9) has been used. (7.7) gives the reflection coefficient at normal incidence, calculated using the Feynman path $(F P)$ method, $\rho_{R}^{F P}$ :

$$
\rho_{R}^{F P}=\left|\frac{n-1}{2 n}\right|^{2}
$$

It is measured by observing the ratio of the counting rates of the similar detectors (assumed to be equally efficient), $D_{1}$ and $D_{2}$ :

$$
\frac{\operatorname{Rate}\left(D_{1}\right)}{\operatorname{Rate}\left(D_{2}\right)}=\frac{\left|\mathcal{A}\left(D_{1}\right)\right|^{2}}{\left|\mathcal{A}\left(D_{2}\right)\right|^{2}}=\frac{\rho_{R}^{F P}}{T_{H S M}^{2}}
$$

The modulus of the transition amplitude of the HSR, $T_{H S M}$ is readily measured by replacing the block of transparent material with a plane specular reflector of known reflectivity.

In should be noticed that $\rho_{R}^{F P}$ differs markedly from the Fresnel prediction for the reflection coefficient at normal incidence:

$$
\rho_{R}^{\text {Fresnel }}=\left|\frac{n-1}{n+1}\right|^{2}
$$

For example, for a glass/vacuum interface, with $n_{\text {glass }}=1.5, \rho_{R}^{F P}=0.028, \rho_{R}^{\text {Fresnel }}=$ 0.040, a difference of $43 \%$. A simple experiment similar to that sketched in Fig 7 could easily discriminate between these predictions. Another interesting difference with respect to the Fresnel formula is related to the minus sign on the RHS of (7.7). This sign, for the Fresnel prediction, is, in fact, ambiguous. Taking the limit as the angle of incidence tends to zero for photons linearly polarised perpendicular to the plane of incidence gives for the reflected amplitude $(n-1) /(n+1)$, whereas the similar limit for photons polarised in the plane of incidence is $-(n-1) /(n+1)$ [41]. Of course, at normal incidence, the terms 'perpendicular' and 'parallel' polarisation become meaningless as the 'plane of incidence' is no longer defined. However, the actual phase shift for reflection at normal incidence is certainly measurable, and the path amplitude calculation, unlike the Fresnel formula, gives the definite prediction, $\pi$, for the phase shift.

This phase shift could be measured by replacing the the detector $D_{2}$ by a suitably placed plane specular mirror and adding an optical attenuator so that the light reflected from this mirror and detected in $D_{1}$, and the light scattered by the transparent medium, 
reflected by the HSR and detected in $D_{1}$ have a similar intensity so as the maximise interference effects for a photon passing along either of the two possible paths. Such an experiment, using a suitably calibrated optical attenuator could also measure the reflection coefficient with a single photon detector. The so-modified apparatus is in fact a Michelson interferometer with one of the specular mirrors relaced by a vacuum/glass interface. The Feynman path amplitude description of a conventional Michelson interferometer is given in the next section.

The result (7.7) for the probabilty amplitude is easily adapted to the case when the vacuum in Fig 7 is replaced by a uniform transparent medium of refractive index $n_{1}$ while the refractive index of the original block of transparent medium is denoted by $n_{2}$. Suitably modifying the photon propagators in (7.2), using (5.18) and calculating the contribution to the probability amplitude from photon paths scattered from the atoms of the medium of refractive index $n_{1}$, gives, instead of (7.7), the expression:

$$
\begin{aligned}
\mathcal{A}\left(D_{2}\right) & =-\tilde{\mathcal{A}}_{1}\left(\frac{n_{2}-1}{2 n_{1} n_{2}}-\frac{n_{1}-1}{2 n_{1}^{2}}\right) \exp \left[i \frac{3 \kappa l_{0}}{2}\right] \\
& =-\tilde{\mathcal{A}}_{1}\left(\frac{n_{2}-n_{1}}{2 n_{1}^{2} n_{2}}\right) \exp \left[i \frac{3 \kappa l_{0}}{2}\right]
\end{aligned}
$$

which may be compared with the Fresnel formula, which gives, instead, for the quantity in the large curved brackets of (7.11): $\pm\left(n_{2}-n_{1}\right) /\left(n_{2}+n_{1}\right)$. The minus sign of the second term in the large curved brackets in the first line of (7.11) results from a reversal of the order of the limits in the $x$ integration over the first half-period zone, since the atoms of the medium of refractive index $n_{1}$ are situated at negative $x$. Setting $n_{2}=n$, $n_{1}=1$ in (7.11) recovers the previous result (7.7). If $n_{1}>n_{2}$ there is no phase shift on reflection in accordance with the well-known result of the classical wave theory of light. If $n_{1}=n_{2} \mathcal{A}\left(D_{2}\right)$ vanishes so there is no back-scattered light in a uniform refractive medium. According to (7.11) this may be interpreted as the result of perfect destructive interference between the path amplitudes of photons back-scattered scattered from atoms with positive and negative $x$.

Interference phenomena closely related to those occuring in 'Newton's Rings' [2] that are extensively discussed in [1] are simply analysed by suitably modifying the $x$ integration limits in (7.6) above. Replacing the block of transparent medium by a thin sheet of thickness $\lambda_{\gamma}^{0}(1+2 p) / 4 n$, where $p$ is a positive integer, gives a reflection coefficient four times larger than in (7.8). If the sheet has a thickness $\lambda_{\gamma}^{0}(1+p) / 2 n, \rho_{R}^{F P}$ vanishes due to perfect destructive interference, in this case, of the path amplitudes corresponding to the scattering of the photon from each atom of the medium comprising the sheet.

\section{Spatio-Temporal Interference Effects in the Michel- son Interferometer}

A schematic layout of a Michelson interferometer [42] is shown in Fig 8. The photon

source, $S$, a single excited atom, is produced at a known time, $t_{0}$. For concretness, as discussed in Section 2 above, this can be taken to be an atom of, say, sodium vapour, 

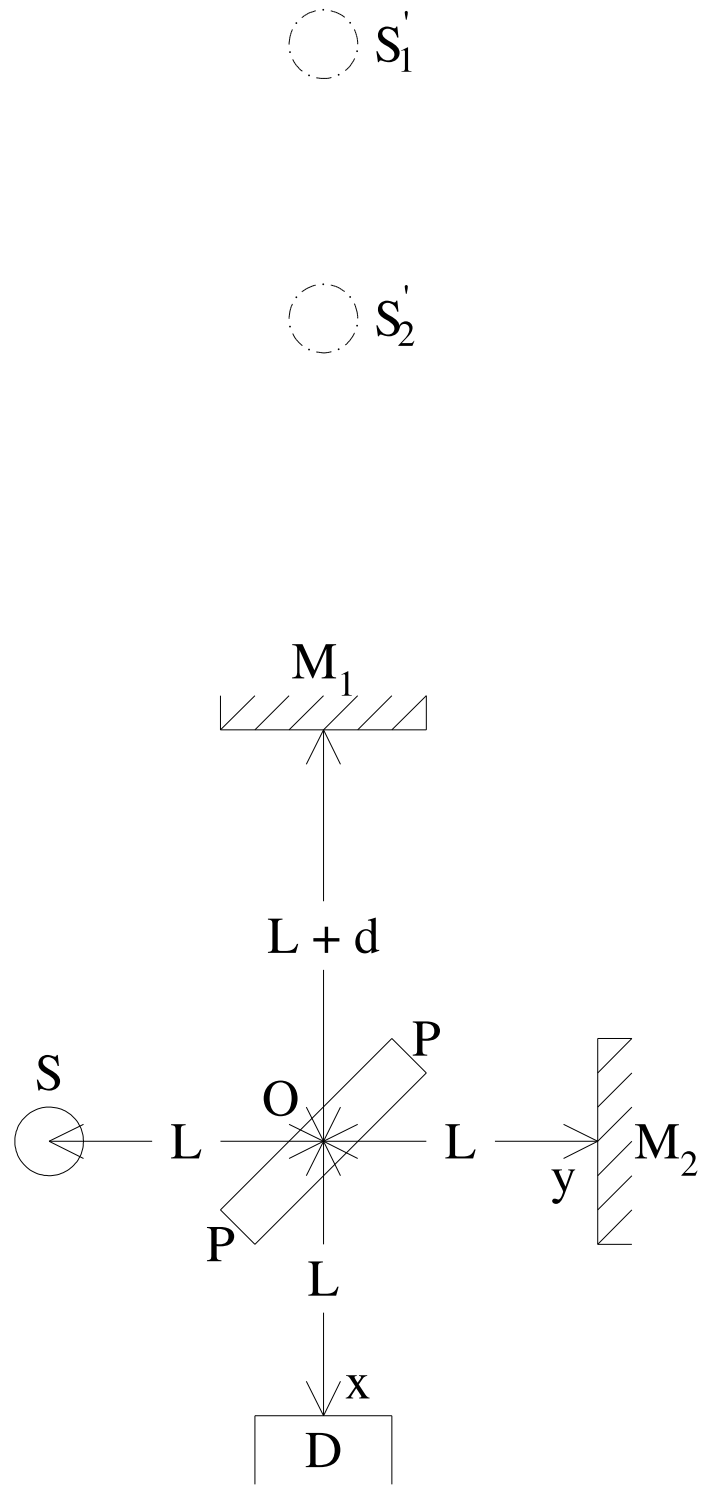

Figure 8: The Michelson interferometer. A photon from the decay of an excited atom at $S$, produced at time $t_{0}$, follows the paths $S O M_{1} O D$ or $S O M_{2} O D$ in alternative histories of the decay process and subsequent space-time propagation of the photon, before being detected at $D$ at time $t_{D} . P P$ is a glass plate half-silvered on its back surface. $S_{1}^{\prime}$ is the virtual image of $S$ after reflection first in $P P$ then in the plane mirror $M_{1} . \quad S_{2}^{\prime}$ is the virtual image of $S$ after reflection first in the plane mirror $M_{2}$ then in PP. The path diffrence $2 d$ is chosen to be $\geq c \tau_{S}$ where $\tau_{S}$ is the mean lifetime of the excited source atom. Note that the atom must decay at different times in its alternative histories in order that the photon arrives at the detector at the fixed time $t_{D}$. It is just this time difference, determining the phase advance of the propagator of the excited atom, that determines the size of the quantum interference term generated by the amplitudes corresponding to the two alternative paths. 
resonantly excited by a tuned, pulsed, laser beam. The pulse width should be at most a few nanoseconds and the time of passage should be known with a precision of 1ns or better.

The photon produced by decay of the excited atom may follow the paths $S O M_{1} O D$ (reflection in by the mirror $M_{1}$ ) or $S O M_{2} O D$ (reflection in by the mirror $M_{2}$ ) before being detected, at time $t_{D}$, in the photon detector at $D$. The glass plate PP situated at the origin of a Cartesian coordinate system, contains the $z$-axis of the latter and is inclined at $45^{\circ}$ to the $x$ - and $y$-axes. The back face of the plate is partially silvered to increase the moduli of the path amplitudes associated with the paths $S O M_{1} O D$ and $S O M_{2} O D$. In a practical interferometer, focussing lenses would be installed in the paths $S O$ and $O D$ to render the photon trajectories in the interferometer parallel to the $x$ - and $y$-axes and increase the efficiency of photon detection. They would contribute a constant multiplicative factor to the interfering path amplitudes and so have no effect on the interference phenomena discussed here.

The simplest way to understand and analyse the operation of the interferometer is to consider the virtual images $S_{1}^{\prime}$ and $S_{2}^{\prime}$ of the source, as reflected in the partially silvered plate and the mirrors $M_{1}$ and $M_{2}$, respectively, and viewed from the position of the photon detector [42] (see Fig 8). The following length intervals are defined:

$$
S O=O M_{2}=O D \equiv L, \quad O M_{1} \equiv L+d
$$

It then follows that:

$$
S_{1}^{\prime} D \equiv L_{1}=4 L+2 d, \quad S_{2}^{\prime} D \equiv L_{2}=4 L
$$

The probability amplitudes, $\mathcal{A}(1), \mathcal{A}(2)$ for photons reflected at $M_{1}, M_{2}$ and detected by $\mathrm{D}$ at time $t_{D}$ are given by (5.18) as:

$$
\begin{aligned}
& \mathcal{A}(1)=\frac{\tilde{\mathcal{A}}_{1}}{L_{1}} \exp \left[-i\left(\kappa c-\frac{i}{2 \tau_{S}}\right)\left(t_{D}-t_{0}-\frac{L_{1}}{c}\right)\right] \\
& \mathcal{A}(2)=\frac{\tilde{\mathcal{A}}_{2}}{L_{2}} \exp \left[-i\left(\kappa c-\frac{i}{2 \tau_{S}}\right)\left(t_{D}-t_{0}-\frac{L_{2}}{c}\right)\right]
\end{aligned}
$$

where the the space-time independent amplitudes $\tilde{\mathcal{A}}_{1}$ and $\tilde{\mathcal{A}}_{2}$ include the production and detection process amplitudes as well as the amplitudes describing all transmission and reflection processes in the arms of the interferometer. In (8.1) and (8.2) the mean life $\tau_{S}=\hbar / \Gamma_{S}$ of the source atom has been introduced as in (3.12). For the Sodium D-lines $\tau_{S} \simeq 10 \mathrm{~ns}$. This value will be taken for the quantitative predictions presented below. Using (2.1) and (2.3), the probability, $P\left(t_{D}<t_{D}^{\max }\right)$ to detect the photon in $\mathrm{D}$ during the time interval $t_{0}<t_{D}<t_{D}^{\max }$ is given by the following expressions:

(i) For: $t_{D}^{\max } \leq t_{0}+L_{2} / c$

$$
P\left(t_{D}<t_{D}^{\max }\right)=0
$$

(ii) For: $t_{0}+L_{2} / c<t_{D}^{\max } \leq t_{0}+L_{1} / c$ 


$$
P\left(t_{D}<t_{D}^{\max }\right)=\int_{t_{0}+L_{2} / c}^{t_{D}^{\max }}|\mathcal{A}(2)|^{2} d t_{D}=\frac{\tau_{S}\left|\tilde{\mathcal{A}}_{2}\right|^{2}}{L_{2}^{2}}\left[1-\exp \left[-\frac{1}{\tau_{S}}\left(t_{D}^{\max }-t_{0}-\frac{L_{2}}{c}\right)\right]\right]
$$

(iii) For: $t_{0}+L_{1} / c<t_{D}^{\max }$

$$
\begin{aligned}
P\left(t_{D}<t_{D}^{\max }\right) & =\int_{t_{D}^{\min }}^{t_{D}^{\max }}|\mathcal{A}(1)+\mathcal{A}(2)|^{2} d t_{D} \\
& =\int_{t_{0}+L_{2} / c}^{t_{D}^{\max }}|\mathcal{A}(2)|^{2} d t_{D}+\int_{t_{0}+L_{1} / c}^{t_{D}^{\max }}\left(|\mathcal{A}(1)|^{2}+2 \operatorname{Re}\left[\mathcal{A}(1) \mathcal{A}(2)^{*}\right]\right) d t_{D} \\
& =\tau_{S}\left\{\frac{\left|\tilde{\mathcal{A}}_{2}\right|^{2}}{L_{2}^{2}}\left[1-\exp \left[-\frac{1}{\tau_{S}}\left(t_{D}^{\max }-t_{0}-\frac{L_{2}}{c}\right)\right]\right]\right. \\
& +\left[1-\exp \left[-\frac{1}{\tau_{S}}\left(t_{D}^{\max }-t_{0}-\frac{L_{1}}{c}\right)\right]\right] \\
& \times\left[\frac{\left|\tilde{\mathcal{A}}_{1}\right|^{2}}{L_{1}^{2}}+2 \frac{\left|\tilde{\mathcal{A}}_{1}\right|\left|\tilde{\mathcal{A}}_{2}\right|}{L_{1} L_{2}} \exp \left(-\frac{\left(L_{1}-L_{2}\right)}{2 c \tau_{S}}\right)\right. \\
& \left.\left.\times \cos \left\{\kappa\left(L_{1}-L_{2}\right)+\phi_{12}\right\}\right]\right\}
\end{aligned}
$$

where

$$
\phi_{12}=\operatorname{phase}\left(\tilde{\mathcal{A}}_{1}\right)-\operatorname{phase}\left(\tilde{\mathcal{A}}_{2}\right)
$$

For condition (i) the photon can arrive at the detector by neither path, so its detection probability vanishes. For condition (ii) the photon can arrive at the detector only via the path $\mathrm{SOM}_{2} O D$ so that there is no interference phenomenon. For condition (iii) the photon may arrive at the detector via either path so interference is possible. With the aid of optical compensators in the arms $O M_{1}, O M_{2}, \tilde{\mathcal{A}}_{1}$ and $\tilde{\mathcal{A}}_{1}$ may be chosen so that:

$$
\frac{\tilde{\mathcal{A}}_{1}}{L_{1}}=\frac{\tilde{\mathcal{A}}_{2}}{L_{2}}=K
$$

In this case (also setting $t_{0}=0$ ) (8.5) simplifies to :

$$
\begin{aligned}
P\left(t_{D}<t_{D}^{\max }\right) & =\tau_{S} K^{2}\left\{2-f\left(t_{D}^{\max }\right)\left(1+e^{\frac{2 d}{c \tau_{S}}}\right)\right. \\
& \left.+2\left(e^{-\frac{d}{c \tau_{S}}}-f\left(t_{D}^{\max }\right) e^{\frac{d}{c \tau_{S}}}\right) \cos \left(2 \kappa d+\phi_{12}\right)\right\}
\end{aligned}
$$

where

$$
f(t) \equiv \exp \left[-\frac{1}{\tau_{S}}\left(t-\frac{4 L}{c}\right)\right]
$$

Neglecting small corrections due to the exponential terms in (8.7) the maximum and minimum values of $P\left(t_{D}<t_{D}^{\max }\right)$ are:

$$
\begin{aligned}
P_{\max (\min )} & =\tau_{S} K^{2}\left\{2-f\left(t_{D}^{\max }\right)\left(1+e^{\frac{2 d}{\tau_{S}}}\right)\right. \\
+ & \left.(-) 2\left(e^{-\frac{d}{c \tau_{S}}}-f\left(t_{D}^{\max }\right) e^{\frac{d}{c \tau_{S}}}\right)\right\}
\end{aligned}
$$

and the 'fringe visibility' $V\left(t_{D}<t_{D}^{\max }\right)$ is:

$$
V\left(t_{D}<t_{D}^{\max }\right)=\frac{P_{\max }-P_{\min }}{P_{\max }+P_{\min }}=\frac{2\left(e^{-\frac{d}{c \tau} S}-f\left(t_{D}^{\max }\right) e^{\frac{d}{c \tau}}\right)}{2-f\left(t_{D}^{\max }\right)\left(1+e^{\frac{2 d}{c \tau} S}\right)}
$$


Curves of $V\left(t_{D}<t_{D}^{\max }\right)$ as a function of $t_{D}^{\max }$ are presented in Fig 9 for: $\tau_{S}=10 \mathrm{~ns}$, $L=50 \mathrm{~cm}$ and $d=12.5,25$ and $50 \mathrm{~cm}$. Such interference effects as a function of $t_{D}^{\max }-t_{0}$, are readily observable. It is sufficient to adjust the interferometer for maximum destructive or constructive interference near some fixed value $d=d_{0}$ and measure $V$, by small variations of $d$ around $d_{0}$, for different values of $t_{D}^{\max }$. If $N_{\max }\left(N_{\min }\right)$ are the numbers of photons recorded with $t_{D}<t_{D}^{\max }$ when the interferometer is adjusted for maximum constructive (destructive) interference then:

$$
V\left(t_{D}<t_{D}^{\max }\right)_{\exp }=\frac{N_{\max }-N_{\min }}{N_{\max }-N_{\min }}
$$

which may be compared with the prediction of (8.9). Fitting this prediction to the data determines the the only parameter, $\tau_{S}$, that is not fixed by the geometry of the experiment.

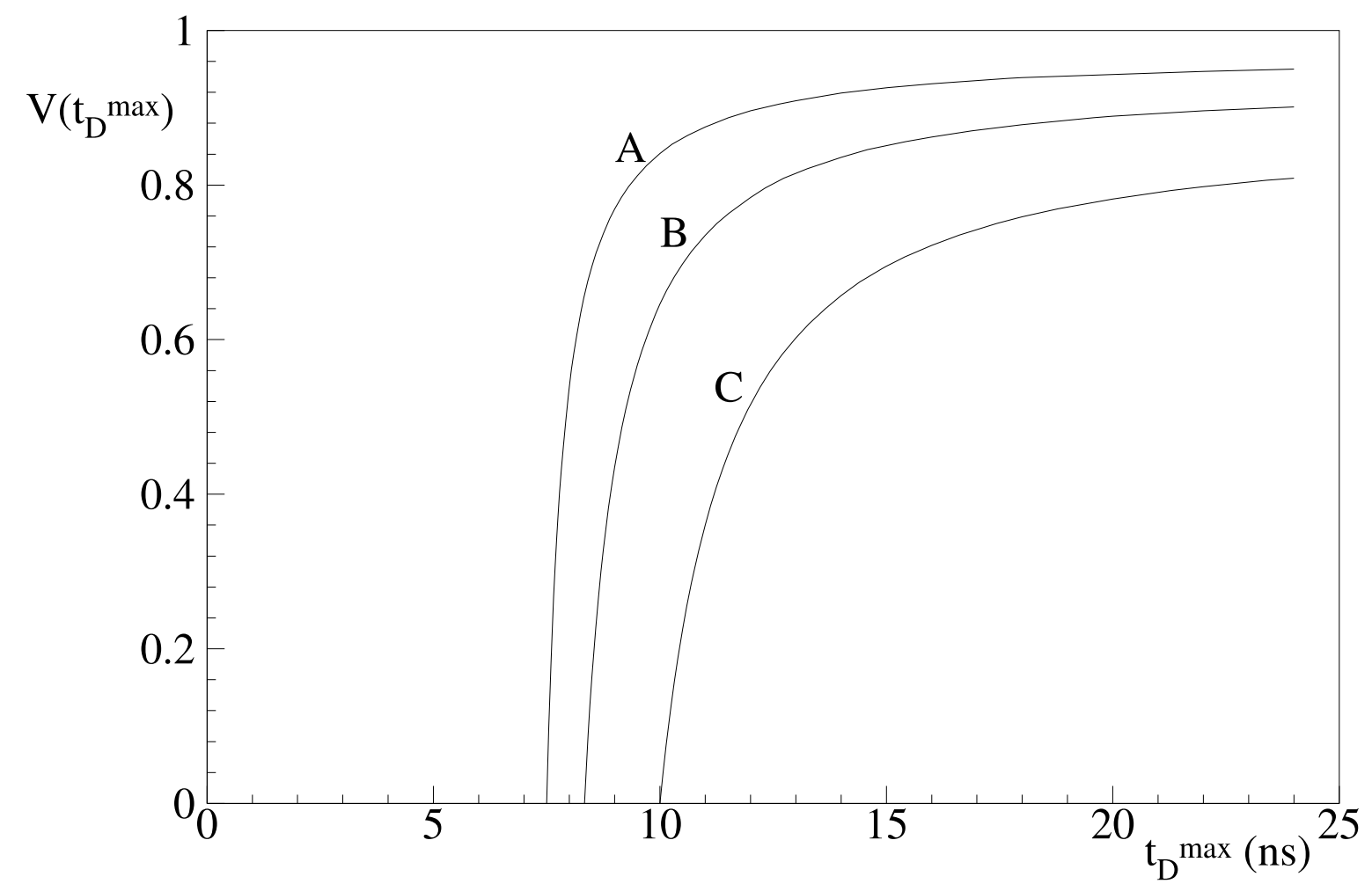

Figure 9: Fringe visibilty $V$ in the Michelson interferometer of Fig.8 as a function of $t_{D}^{\max }$ the maximum observation time of the photon at $D$. The excited source atom is produced at time $t=0 . L=50 \mathrm{~cm}$ and $\tau_{S}=10 \mathrm{~ns}$. The curves $A, B$ and $C$ correspond to $d=12.5,25$ and $50 \mathrm{~cm}$.

Choosing a value of $t_{D}^{\max } \gg c \tau_{S}$ results in $f\left(t_{D}^{\max }\right) \simeq 0$. The time-integrated fringe visibility as a function of $d, V_{\infty}(d)$, is then qiven by (8.9) as

$$
V_{\infty}(d)=V\left(t_{D}<\infty\right)=\exp \left(-\frac{d}{c \tau_{S}}\right)
$$

Thus the time integrated visiblity is predicted to decrease exponentially with the path difference $2 d$. Exactly the same behaviour is predicted by the classical wave theory of light [43]. In this calculation, similar to that of Michelson [44], The visibility was calculated using the equation obtained by making the substitutions $\kappa \rightarrow 2 \pi c \nu_{\gamma}$ and 
$f\left(t_{D}^{\max }\right) \gg c \tau_{S}$ in (8.7) above, and by weighting the cosine interference term with a Lorentzian distribution in the freqency, $\nu_{\gamma}$, of the photon. In the Feynman path amplitude calculation $\kappa$ is defined by the pole masses of the initial and final states (see (4.9) above), which, unlike the photon frequency, do not vary on an event-by-event basis. Thus, from the point-of-view of the Feynman path amplitude calculation, the classical wave calculation is incorrect, even though it predicts the same result.

In the above treatment, two potentially important physical effects have been neglected.

The first effect is 'pressure broadening' of the atomic linewidth. This results in an observed value of $\tau_{S}$ less then that, $\tau_{S}^{\text {nat }}$, corresponding to the 'natural' linewidth of an isolated, freely decaying, atom:

$$
\frac{1}{\tau_{S}}=\frac{1}{\tau_{S}^{n a t}}+\frac{1}{\tau_{P}}
$$

where $\tau_{P}$ is a characteristic lifetime parameter that tends to infinity as the pressure of the source of excited atoms tends to zero. The physical origin of this effect is easily understood in the path amplitude language. In order for the source atom to retain the coherent phase relationship between different path amplitudes, following from (4.3), that is assumed in (8.1) and (8.2) above, the atom must remain in the excited state sufficiently long that unhindered spontaneous decay can occur. If this is not the case, for example, if the atom is dexcited, or excited into a different state by inter-atomic collisions, after the time corresponding to the 'earlier' path amplitude but before the time corresponding to the 'later' one, no interference will be possible. In the case that all inter-atomic collisions destroy the excited state then the parameter $\tau_{P}$ is simply related to the mean time between such collisions. There is then competition between decay and inter-atomic collisions for destruction of the excited state. On the assumption that these are independent processes:

$$
\Gamma_{S}=\Gamma_{S}^{n a t}+\Gamma_{P}
$$

Which is equivalent to (8.12) above. In fact, the actual situation is much more complicated, since the inter-atomic collisions do not always result in destruction of the excited atom and may modify thee energy level or the decay probability of the excited atom. Many attempts were made during the '30s and ' $40 \mathrm{~s}$ of the last century to calculate $\Gamma_{P}$ from phenomenological atomic models $[45,46]$.

The second effect is the possible correction due to motion of the source atom, which has previously been assumed, throughout the present paper, to be at rest. An analogous calculation has recently been performed [12] by the present author for the related 'neutrino oscillation' problem. It is adapted to the present case in Appendix E below. The result is markedly different from 'Doppler effect' formulae found in the previous literature and text books. There is no damping effect at any order in $v / c$, only a phase shift. The classical wave theory of light predicts a Gaussian dependence of the fringe visiblity on the path difference due to the first order Doppler Effect (DE):

$$
V_{\infty}(d)^{D E}=\exp \left[-\pi\left(\frac{2 \pi d}{\lambda_{\gamma}^{0}}\right)^{2} \frac{k T}{M}\right]
$$

This formula, due to Lord Rayleigh, is obtained by weighting the cosine interference term in the visibility function by a Gaussian distribution of $\nu_{\gamma}$ derived, using the Doppler 


\begin{tabular}{|c|ccccccc|}
\hline Transition & $\lambda_{\gamma}^{0}(\AA)$ & $\Delta^{e x p}(\mathrm{~cm})$ & $\Delta^{D E}(\mathrm{~cm})$ & $\Delta^{\text {nat }}(\mathrm{cm})$ & $\tau_{S}(\mathrm{~ns})$ & $\tau_{S}^{\text {nat }}(\mathrm{ns})$ & $\tau_{P}(\mathrm{~ns})$ \\
\hline $\mathrm{H}_{r} 3 \mathrm{p}-2 \mathrm{~s}$ & 6563 & 19.0 & 14.3 & 225 & 0.46 & 5.4 & 0.50 \\
$\mathrm{H}_{b} 4 \mathrm{p}-2 \mathrm{~s}$ & 4861 & 8.5 & 10.6 & 516 & 0.204 & 12.4 & 0.207 \\
$\mathrm{Na}$ D 3p-3s & 5893 & 80.0 & 65.0 & 225 & 1.92 & 12.4 & 2.98 \\
\hline
\end{tabular}

Table 1: Measurements from [44] of path lengths $\Delta^{\exp }$ yielding a fringe visiblity of $50 \%$ in comparison with theoretical expectations $\Delta^{\text {nat }}$ derived using (8.11) and $\Delta^{D E}$ from (8.14). The corresponding values of the lifetime prameters $\tau_{S}$ and $\tau_{S}^{\text {nat }}$ are also shown as well as the pressure-broadening parameter $\tau_{P}$ derived from (8.12).

effect, from a Maxwellian distribution of source-atom velocities. As in the classical wave derivation of (8.11) this requires the substitution $\kappa \rightarrow 2 \pi c \nu_{\gamma}$ in (8.7) above, which is incorrect for the Feynman path amplitude calculation.

The prediction of (8.14) was compared by Michelson in [44] to a number of experimental measurements of fringe visiblity. The results obtained for some transitions in Hydrogen and Sodium are summarised in Table 1. The experimental observable, $\Delta$, is the path difference $2 d$ in the Michelson interferometer for which $V_{\infty}\left(\frac{\Delta}{2}\right)=0.5$. Also shown in Table 1 are the measured values of $\tau_{S}=\Delta /(2 c \ln 2)$ derived from $(8.11), \Delta^{D E}$ from (8.14), theoretical values of $\tau_{S}^{\text {nat } 24}$ and values of the pressure-broadening lifetime $\tau_{P}$ calculated from $\tau_{S}$ and $\tau_{S}^{n a t}$ using (8.12). At the time that Michelson performed the measurements shown in Table 1, Quantum Mechanics had yet to be invented so no theoretical values of $\tau_{S}^{\text {nat }}$ were available. The values of $\tau_{P}$ shown in Table 1 show that pressure-broadening effects are very important for the Hydrogen lines, but less so for the Sodium D-lines. Michelson had observed that line widths increase with increasing pressure, but compared his measurements only with the Doppler formula (8.14). For this comparison, in the case of the Hydrogen lines, Michelson chose a temperature of $485^{\circ} \mathrm{K}$ in (8.14) corrsponding to a R.M.S. velocity, $\sqrt{k T / M}$, of $2.0 \times 10^{3} \mathrm{~m} / \mathrm{sec}$. Equation (8.14) was used to predict values of $\Delta / \lambda_{\gamma}^{0}$ for different atomic transitions, which were compared with the corresponding experimental quantities. Rough agreement was claimed although some positive deviations of up to $30 \%$ and negative deviations up to $40 \%$ were observed in some cases. No uncertainties were quoted on the experimental measurements. Given the importance of pressure broadening effects for the measurements presented in [44], and the difficulty to distinguish between the former effect and that due to source motion, these measurements provide no evidence for or against the possiblity of a vanishingly small source-motion correction, as predicted by the path amplitude calculation.

Precise measurement of the temperature dependence of the line widths of excited atoms in a gaseous source then constitutes another stringent experimental test of path amplitude predictions. In practice it was very difficult in the past to disentangle conjectured 'Doppler effect' and actual 'pressure broadening' effects in the data. Both are expected to result in larger linewidths at higher temperatures for a constant volume gaseous source. One possible approach is to measure $\tau_{S}$ in a Michelson interferometer, using a constant temperature gaseous source, as a function of pressure at low pressure. Extrapolating to

\footnotetext{
${ }^{24}$ The values of $\tau_{S}^{n a t}$ for the hydrogen lines are taken from P136 of [47]. The value for the sodium D-lines is calculated using the formula: $1 / \tau_{S}^{n a t}=4 \pi r_{e} \nu_{m n} 2 f /(3 c)$ [48] where $r_{e}$ is the classical electron radius and $f=0.9755$.
} 
zero pressure then gives a line width with contributions only from the natural width and possible source motion effects, as $\tau_{P}$ is infinite at zero pressure. The prediction of the Feynman path amplitude calculation is that the same extrapolated value of $\tau_{S}$ should be obtained, by this procedure, independently of the temperature of the source.

\section{Spatially Dependent Interference Effects in Quan- tum Systems with Two Probability Amplitudes.}

In this section the Feynman path amplitude description will be applied to some analogous physical systems: the Young double-slit experiment (YDSE) using either photons or electrons, quark flavour oscillations in the neutral kaon system and neutrino oscillations. In all cases the total probability amplitude for the system, $A_{F I}$, decomposes into the sum of two probability amplitudes, $A_{F I}^{A}$ and $A_{F I}^{B}$ :

$$
A_{F I}=A_{F I}^{A}+A_{F I}^{B}=\left|A_{F I}^{A}\right| e^{i \phi_{A}}+\left|A_{F I}^{B}\right| e^{i \phi_{B}}
$$

For the YDSE experiments, $A_{F I}^{A}$ and $A_{F I}^{B}$ describe experiments where either one of the slits is closed. For the neutral kaon or neutrino oscillation experiments, these amplitudes correspond to flavour mixing scenarios where only a single mass eigenstate is produced. The experimental observable, $P_{F I}$, is the probability to observe one of the set of states $\mathrm{F}:\left|f_{m}\right\rangle, m=1,2, \ldots$ given a prepared state in the set $\mathrm{I}:\left|i_{l}\right\rangle, \quad l=1,2, \ldots$ (see Section 2 above). According to Feynman's principles I and III ((2.1) and (2.3)):

$$
\begin{aligned}
P_{F I} & =\left|A_{F I}^{A}+A_{F I}^{B}\right|^{2} \\
& =\left|A_{F I}^{A}\right|^{2}+\left|A_{F I}^{B}\right|^{2}+2 \operatorname{Re}\left[A_{F I}^{A}{ }^{*} A_{F I}^{B}\right] \\
& =\left|A_{F I}^{A}\right|^{2}+\left|A_{F I}^{B}\right|^{2}+2\left|A_{F I}^{A}\right|\left|A_{F I}^{B}\right| \cos \left(\phi_{B}-\phi_{A}\right)
\end{aligned}
$$

The interesting physical phenomenon, in every case, is described by the interference term in (9.2) that depends on the phase difference $\phi_{B}-\phi_{A}$. The origin of the latter is different in all the four examples under discussion. In a YDSE, the phase difference is a consequence of different path lengths corresponding to the two probability amplitudes just as for the Michelson interferometer discussed in the previous section. However the physical mechanism that actually generates the phase difference is quite different in the two cases. For the photon experiment it is the space-time propagator of the excited source atom. For the electron experiment it is the space-time propagator of the electron itself. For neutral kaon oscillations the path difference is constant and the phase difference is a result of the different space-time propagators of the $\mathrm{K}_{S}$ and $\mathrm{K}_{L}$ mesons. The propagators are different because of the different masses of these states. As previously pointed out $[11,12,13]$, for the case of neutrino oscillations, both the propagator of the unstable source particle whose decay produces the neutrino, and the propagators of the neutrino mass eigenstates $\nu_{1}$ and $\nu_{2}$ give important contributions to the phase difference. In each case a realistic experiment is described in which all relevant physical parameters are discussed. In most previous discussions only simplifed models have been used. These models give essentially the same results as the path amplitude calculations for both the YDSE as well as for neutral kaon oscillations, but a markedly different one for neutrino oscillations. 


\section{Young Double Slit Experiment with Photons}

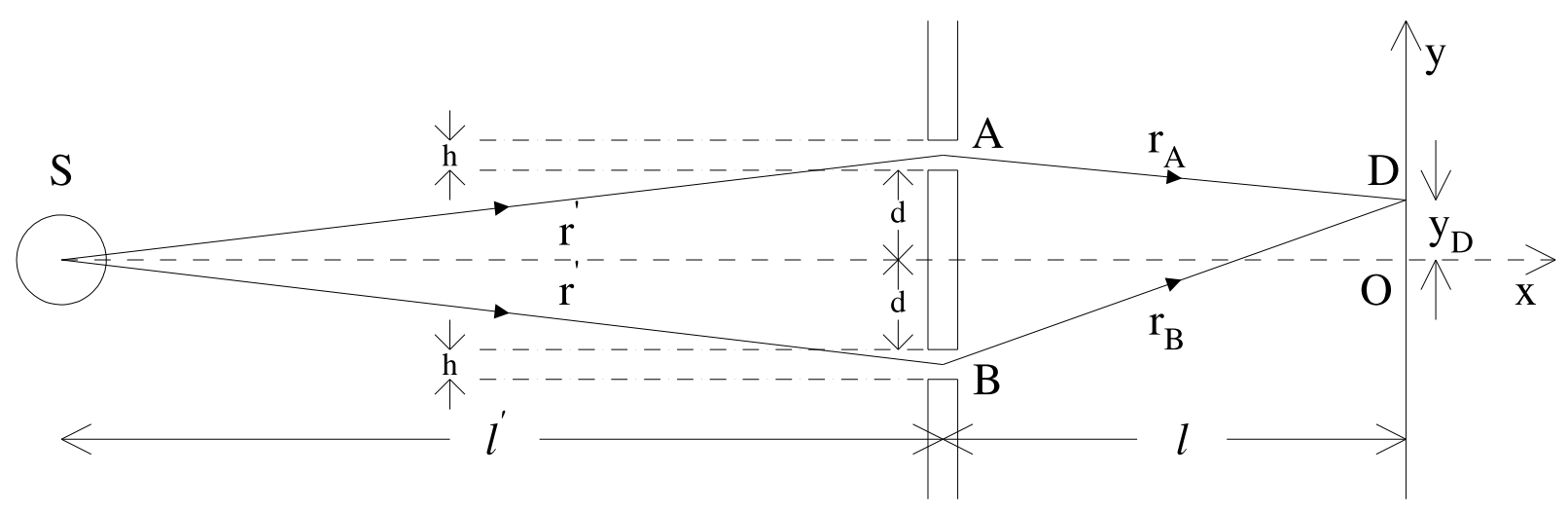

Figure 10: Geometry of a Young double slit experiment. Particles are produced at the source $S$ and detected at $D$. For the photon experiment, $S$ is a single excited atom. For electrons $S$ is the exit of an electrostatic accelerator producing a narrow electron beam with the Gaussian momentum profile of (9.13).

A schematic layout of a typical YDSE is shown in Fig.10, where the important geometrical parameters are defined. The photon source is a single excited atom at $\mathrm{S}$. The photon passes through the slits $\mathrm{A}$ or $\mathrm{B}$ in correspondence with the probability amplitudes $A_{D S}^{A}\left(t_{D}\right)^{\gamma}$ or $A_{D S}^{B}\left(t_{D}\right)^{\gamma}$, and is detected at $\mathrm{D}$ at time $t_{D}$. Fig.10 shows the projections of the alternative paths of the photon in the $x y$ plane, which is perpendicular to the long edges of the slits. In order for the photon to arrive at D it must be Fresnel diffracted, in the $x y$ plane, at one of the slits. It is assumed that the height, $h$, of the slits is sufficiently small for this to occur, and also that the y-dependence of the detection rate, resulting from diffraction (i.e. due to integrating the contributions corresponding to paths passing at different positions within the slit) may be neglected for small values of $y_{D}$ (see Fig. 10). The widths of the slits, $w$, are assumed to be sufficiently long that the photon follows an essentially rectilinear trajectory in the $x z$ and $y z$ planes. The probability amplitudes $A_{D S}^{A}\left(t_{D}\right)^{\gamma}$ and $A_{D S}^{B}\left(t_{D}\right)^{\gamma}$ are given directly by (4.8) above (compare Fig.1 and Fig.10):

$$
\begin{aligned}
& A_{D S}^{A}\left(t_{D}\right)^{\gamma}=\frac{\tilde{\mathcal{A}}}{r^{\prime} r_{A}} \mathcal{A}_{\text {diff }} h w \exp \left[-\frac{i}{\hbar}\left(\kappa c-i \frac{1}{2 \tau_{S}}\right)\left(t_{D}-t_{0}-\frac{r_{A}+r^{\prime}}{c}\right)\right] \\
& A_{D S}^{B}\left(t_{D}\right)^{\gamma}=\frac{\tilde{\mathcal{A}}}{r^{\prime} r_{B}} \mathcal{A}_{\text {diff }} h w \exp \left[-\frac{i}{\hbar}\left(\kappa c-i \frac{1}{2 \tau_{S}}\right)\left(t_{D}-t_{0}-\frac{r_{B}+r^{\prime}}{c}\right)\right]
\end{aligned}
$$

Neglecting the angular dependence of $\mathcal{A}_{\text {diff }}$ and setting $r_{A}=r_{B}=l$ in the denominator of the RHS of (9.3) and (9.4) (a good approximation for small values of $y_{D}$ ), substituting the path amplitudes in (9.2) and integrating, with suitable limits, over $t_{D}$, as done above the the similar case of the Michelson interferometer, gives the result, similar to (8.7) above:

$$
P_{F I} \equiv P_{F I}\left(t_{D}<\infty\right)=2 \tau_{S}\left|A_{0}^{\gamma}\right|^{2}\left\{1+\exp \left[-\frac{\left(r_{B}-r_{A}\right)}{2 c \tau_{S}}\right] \cos \kappa\left(r_{B}-r_{A}\right)\right\}
$$

where

$$
A_{0}^{\gamma} \equiv-\frac{i \tilde{\mathcal{A}} \kappa h w}{2 \pi l r^{\prime}}
$$


Assuming that $y_{D}, d \ll l$, it follows from the geometry of Fig.11 that:

$$
\Delta r \equiv r_{B}-r_{A} \simeq \frac{2\left(d+\frac{h}{2}\right) y_{D}}{l}
$$

giving in (9.5):

$$
\begin{aligned}
P_{F I}\left(y_{D}\right) & =2 \tau_{S}\left|A_{0}^{\gamma}\right|^{2}\left[1+\exp \left[-\frac{\left(d+\frac{h}{2}\right) y_{D}}{c \tau_{S} l}\right] \cos \left(\frac{2 \kappa\left(d+\frac{h}{2}\right) y_{D}}{l}\right)\right] \\
& \simeq 4 \tau_{S}\left|A_{0}^{\gamma}\right|^{2} \cos ^{2}\left(\frac{2 \pi\left(d+\frac{h}{2}\right) y_{D}}{\lambda_{\gamma}^{0} l}\right)
\end{aligned}
$$

where in the last line of (9.8) the small exponential damping correction to the interference term has been neglected. This is identical with the well-known result derived using the classical wave theory. The spacing, $\Delta y_{D}$, between adjacent interference fringes is give by (9.8) as:

$$
\Delta y_{D}=\frac{\lambda_{\gamma}^{0} l}{2\left(d+\frac{h}{2}\right)}
$$

For typical experimental values: $l=10 \mathrm{~cm}, d+h / 2=1 \mathrm{~mm}, \lambda_{\gamma}^{0}=5893 \AA, \Delta y_{D}$ is 29 $\mu m$. For the Sodium-lines $\tau_{S}^{n a t}=5.4 \mathrm{~ns}$, so that the damping correction in (9.5) is only $\exp \left(-1.8 \times 10^{-11} n\right)$ for the $n$th interference fringe.

\section{Young Double Slit Experiment with Electrons}

The actual realisation of Feynman's gedankenexperiment, discussed at length in chapter 1 of Vol III of [3] was not done until the early 1980s [50]. A closely analagous experiment was performed by Tonomura et al [49]. In this electron biprism experiment, the double slits of a YDSE are replaced by a region of electric field around a thin wire that provides an equivalent deflection of the electron trajectories to that provided by Fresnel diffraction at the slits in a YDSE.

In electron quantum interference experiments the electrons are typically produced by a 'direct current' source using thermionic of field emission. There is thus no source with a well-defined quantum state yielding a known time-dependent phase as in the optical experiments considered above, or the neutrino oscillation experiments to be discussed below.

Referring to Fig.10 and using (3.11) for the space-time propagator of the electron, the probability amplitudes analagous to (9.3) and (9.4) for the electron case are, on making similar geometrical approximations to those made in (9.5) above:

$$
\begin{aligned}
& A_{D S}^{A e}=A_{0}^{e} \int \exp \left[-\frac{i}{\hbar}\left[E_{e} \Delta t_{A}-p_{e}\left(r^{\prime}+r_{A}\right)\right]\right] f\left(p_{e}\right) d p_{e} \\
& A_{D S}^{B e}=A_{0}^{e} \int \exp \left[-\frac{i}{\hbar}\left[E_{e} \Delta t_{B}-p_{e}\left(r^{\prime}+r_{B}\right)\right]\right] f\left(p_{e}\right) d p_{e}
\end{aligned}
$$

where

$$
A_{0}^{e} \equiv \frac{A_{D} \beta_{e}^{2} \mathcal{A}_{\text {diff }} h w A_{S}}{r^{\prime} l}
$$


In these equations, $A_{S}$ is the electron production amplitude, $\mathcal{A}_{\text {diff }}$, the amplitude for diffraction at a slit and $A_{D}$ the amplitude for the electron detection process. The function $f\left(p_{e}\right)$ is the normalised distribution of electron momenta at the detector. In the following a Gaussian form will be used:

$$
f(p)=\frac{1}{\sqrt{2 \pi} \sigma_{p}} \exp \left[-\frac{(p-\langle p\rangle)^{2}}{2 \sigma_{p}^{2}}\right]
$$

It can be seen that the two important differences between a photon and an electron YDSE. are that, (i) in the electron case the phases of the probability amplitudes are determined by the space-time propagator of the electron, whereas in the photon case the phase is determined entirely by the coherent source, the contribution from the photon propagator vanishing, and (ii) unlike on-shell photons, the electrons do not have a constant velocity, so that their actual velocity or momentum distribution must be taken into account in order to construct the probability amplitudes (9.10) and (9.11). Because of the photon's constant velocity it must be produced at different times in amplitudes corresponding to paths of different lengths. However, the electron can be produced at the same time in both probability amplitudes (i.e. $\Delta t_{A}=\Delta t_{B}$ in (9.10) and (9.11)) and produce the same detection event, at a well defined time, due to different velocities along each path. In this case, unlike for the photon YDSE above and neutrino oscillations, to be discussed below, there is no contribution to the interference phase from the production amplitude $A_{S}$.

The Lorentz-invariant character of the phase of the space-time propagator of a particle (see (3.11) above) enables the phases of the complex exponentials in (9.10) and (9.11) to be written in the following four equivalent ways:

$$
\phi=-\frac{m c^{2} \tau}{\hbar}=-\frac{m c^{2} t}{\gamma \hbar}=-\frac{\left(m c^{2}\right)^{2} r}{E v \hbar}=-\frac{(m c)^{2} r}{p \hbar}
$$

Where $\gamma$ is the usual relativistic parameter $1 / \sqrt{1-\beta^{2}}, \beta \equiv v / c=p c^{2} / E=r /(c t)$. Substituting the appropriate values of $r$ and $p$ then gives for the phase difference in (9.2):

$$
\phi_{B}-\phi_{A}=\frac{\left(m c^{2}\right)^{2}}{c^{2} \hbar}\left(\frac{r^{\prime}+r_{A}}{p_{A}}-\frac{\left(r^{\prime}+r_{B}\right)}{p_{B}}\right)
$$

For equal production times of the electron in the paths $\mathrm{A}$ and $\mathrm{B}$ the condition:

$$
t=t_{A}=t_{B}=\frac{r^{\prime}+r_{A}}{v_{A}}=\frac{r^{\prime}+r_{B}}{v_{B}}
$$

is respected. It is shown in Appendix F that, in this case, the RHS of (9.15) may be written, to first order in the small quantity $\Delta r / r^{\prime}=\left(r_{B}-r_{A}\right) / r^{\prime}$, as:

$$
\phi_{B}-\phi_{A}=\frac{\bar{p} \Delta r}{\hbar}=\frac{2 \pi \Delta r}{\lambda_{e}^{D B}}
$$

where

$$
\bar{p} \equiv \frac{p_{A}+p_{B}}{2}
$$


and

$$
\lambda_{e}^{D B} \equiv \frac{h}{\bar{p}}
$$

Which is de Broglie's formula for the quantum wavelength of a particle. As further discussed in Appendix F, the equal time condition (9.16) is essential to obtain this relation in the Feynman path amplitude approach. The relation (9.16) also implies the following velocity difference for the electron for the paths $\mathrm{A}$ and $\mathrm{B}$ :

$$
v_{B}-v_{A}=\frac{c^{2}\left(m c^{2}\right)^{2}}{\bar{E}^{3}}\left(p_{B}-p_{A}\right)=\frac{\bar{v} \Delta r}{r^{\prime}+\bar{r}}+O\left[(\Delta r)^{2}\right]
$$

where $\bar{E}, \bar{v}$ and $\bar{r}$ are defined similarly to $\bar{p}$ in (9.18).

Taking the modulus squared of (9.10) or (9.11), and performing the momentum integration, gives:

$$
\begin{aligned}
\left|A_{D S}^{A, B} e\right|^{2} & =\left|A_{0}^{e}\right|^{2} \iint e^{i\left(\phi_{A, B}(p)-\phi_{A, B}\left(p^{\prime}\right)\right)} f(p) f\left(p^{\prime}\right) \delta\left(p-p^{\prime}\right) d p d p^{\prime} \\
& =\frac{1}{2 \sqrt{\pi} \sigma_{p}}\left|A_{0}^{e}\right|^{2}
\end{aligned}
$$

The equal time condition gives, for the interference term in (9.2):

$$
\begin{aligned}
I_{A B} & \equiv 2\left|A_{F I}^{A}\right|\left|A_{F I}^{B}\right| \cos \left(\phi_{B}-\phi_{A}\right) \\
& =2\left|A_{0}^{e}\right|^{2} \operatorname{Re}\left\{\iint e^{-\frac{i\left(p_{A}+p_{B}\right) \Delta r}{2 \hbar}} f\left(p_{A}\right) f\left(p_{B}\right) \delta\left(p_{B}-p_{A}-\Delta p\right) d p_{A} d p_{B}\right\} \\
& =\frac{\left|A_{0}^{e}\right|^{2}}{\pi \sigma_{p}^{2}} \operatorname{Re}\left\{\int e^{-\frac{i\left(p_{A}+\frac{\Delta p}{2}\right) \Delta r}{\hbar}} e^{-\frac{\left(p_{A}-\langle p\rangle\right)^{2}}{2 \sigma_{p}^{2}}} e^{-\frac{\left(p_{A}+\Delta p-\langle p\rangle\right)^{2}}{2 \sigma_{p}^{2}}} d p_{A}\right\}
\end{aligned}
$$

where, from (9.20),

$$
\Delta p \equiv p_{B}-p_{A}=\bar{p}\left(\frac{\bar{E}}{m c^{2}}\right)^{2} \frac{\Delta r}{r^{\prime}+\bar{r}}+O\left[(\Delta r)^{2}\right]
$$

The $p_{A}$ integral is readily evaluated by the method of 'completing the square' to yield the result:

$$
I_{A B}=\frac{\left|A_{0}^{e}\right|^{2}}{\sqrt{\pi} \sigma_{p}} \exp \left[-\left(\frac{\Delta p}{2 \sigma_{p}}\right)^{2}\right] \exp \left[-\left(\frac{\sigma_{p} \Delta r}{2 \hbar}\right)^{2}\right] \cos \left(\frac{\langle p\rangle \Delta r}{\hbar}\right)
$$

The first exponential damping term takes into account the fact that a non-vanishing spread in electron momentum is necessary for interference to occur - a consequence of the imposed condition of equal production times for the electron in the two paths. The second exponential describes damping due to the width of the momentum distribution. If it is very wide the probability to satisfy the condition (9.20) becomes very small. Inserting (9.21) and (9.24) in (9.2), as well as the expression (9.7) for $\Delta r$, gives finally:

$$
\begin{aligned}
P_{F I}\left(y_{D}\right)^{e} & =\frac{\left|A_{0}^{e}\right|^{2}}{\sqrt{\pi} \sigma_{p}}\left[1+\exp \left\{-\left[\left(\frac{\Delta p}{2 \sigma_{p}}\right)^{2}+\left(\frac{\sigma_{p} \Delta r}{2 \hbar}\right)^{2}\right]\right\} \cos \left(\frac{2\langle p\rangle(d+h / 2) y_{D}}{l \hbar}\right)\right] \\
& \simeq 2 \frac{\left|A_{0}^{e}\right|^{2}}{\sqrt{\pi} \sigma_{p}} \cos ^{2}\left(\frac{2 \pi(d+h / 2) y_{D}}{\lambda_{e}^{D B} l}\right)
\end{aligned}
$$


where in the last line the typically small interference damping corrections ${ }^{25}$ have been neglected. It can be seem by comparing (9.8) and (9.25) that, in this approximation, photons and electrons with $\lambda_{e}^{D B}=\lambda_{\gamma}^{0}$ are predicted to produce identical interference patterns in geometrically indentical YDSE.

\section{Strange Quark Flavour Oscillations}

In this case the Feynman path amplitude description of a specific experiment [51] will be considered. This is done to ensure that the effects of all possibly relevant physical parameters are properly taken into account in the discussion. In the experiment, $\mathrm{K}_{\mathrm{S}}$ or $\mathrm{K}_{\mathrm{L}}$ mesons were produced via the processes: $\pi^{-} p \rightarrow \Lambda\left(\mathrm{K}_{\mathrm{S}}, \mathrm{K}_{\mathrm{L}}\right)$ by a $1.01 \mathrm{GeV} / \mathrm{c}$ pion beam. The initial state proton was either free within a chemically bound hydrogen atom, or bound in a carbon nucleus, of the polythene/plastic scintillator target. Roughly $50 \%$ of the interactions occured on bound protons. Semi-leptonic decays $\mathrm{K}_{\mathrm{S}}, \mathrm{K}_{\mathrm{L}} \rightarrow \pi^{ \pm} e^{\mp} \nu$ were observed, and their proper time intervals calculated. In this experiment the paths $\mathrm{A}$ and $\mathrm{B}$ in (9.2) correspond to the space-time propagation of the $\mathrm{K}_{\mathrm{S}}$ or $\mathrm{K}_{\mathrm{L}}$ mesons respectively, so in the following the labels A,B are replaced by S,L. It is assumed that either meson is detected at a fixed distance, $L$, from its production point.

The detailed physics underlying the production amplitudes of the mesons is not well understood, but is not important for the description of the flavour oscillation phenomenon. An $s \bar{s}$ quark pair is produced by the strong interaction. The $s$ quark is bound in the $\Lambda$, while the $\bar{s}$ undergoes a flavour-changing weak charged-current interaction which produces one of the mass eigenstates $\left|K_{S}\right\rangle,\left|K_{L}\right\rangle$ of the neutral kaon system. These states contain within their quark substructure both $\bar{s} d\left(\left|\mathrm{~K}_{0}\right\rangle\right)$ and $s \bar{d}\left(\left|\overline{\mathrm{K}}_{0}\right\rangle\right)$ components. Neglecting small CP-violating contributions, $\left|\mathrm{K}_{\mathrm{S}}\right\rangle$ and $\left|\mathrm{K}_{\mathrm{L}}\right\rangle$ are eigenstates of $\mathrm{CP}$ :

$$
\begin{array}{ll}
\left|\mathrm{K}_{\mathrm{S}}\right\rangle=\frac{1}{\sqrt{2}}\left(\left|\mathrm{~K}_{0}\right\rangle-\left|\overline{\mathrm{K}}_{0}\right\rangle\right) \quad \mathrm{CP}=+1 \\
\left.\left|\mathrm{~K}_{\mathrm{L}}\right\rangle=\frac{1}{\sqrt{2}}\left(\left|\mathrm{~K}_{0}\right\rangle+\left|\overline{\mathrm{K}}_{0}\right\rangle\right)\right) & \mathrm{CP}=-1
\end{array}
$$

where the states $\left|\mathrm{K}_{0}\right\rangle$ and $\left|\overline{\mathrm{K}}_{0}\right\rangle$ are related by the CP operator as:

$$
\mathrm{CP}\left|\mathrm{K}_{0}\right\rangle=-\left|\overline{\mathrm{K}}_{0}\right\rangle, \quad \mathrm{CP}\left|\overline{\mathrm{K}}_{0}\right\rangle=-\left|\mathrm{K}_{0}\right\rangle
$$

The $\bar{s}$ quark produced in association with the $\Lambda$ couples only to the $\left|\mathrm{K}_{0}\right\rangle$ component of $\left|\mathrm{K}_{\mathrm{S}}\right\rangle$ and $\left|\mathrm{K}_{\mathrm{L}}\right\rangle$. It then follows from (9.26) and (9.27) that, neglecting CP-violating effects, the $\left|\mathrm{K}_{\mathrm{S}}\right\rangle$ and $\left|\mathrm{K}_{\mathrm{L}}\right\rangle$ production amplitudes are equal:

$$
\left\langle\mathrm{K}_{\mathrm{S}} \Lambda|T| \pi^{-} p\right\rangle=\left\langle\mathrm{K}_{\mathrm{L}} \Lambda|T| \pi^{-} p\right\rangle \equiv A_{P}
$$

The decay processes $\mathrm{K}_{\mathrm{S}}, \mathrm{K}_{\mathrm{L}} \rightarrow \pi^{ \pm} e^{\mp} \nu$ have been found, experimentally, to respect the ' $\Delta S=\Delta Q$ Rule', which is predicted in the Standard Electroweak Model, by consideration of the $\mathrm{W}$-boson exchange diagrams that mediate these transitions. This rule predicts that the $\left|\mathrm{K}_{0}\right\rangle$ components of $\left|\mathrm{K}_{\mathrm{S}}\right\rangle$ and $\left|\mathrm{K}_{\mathrm{L}}\right\rangle$, decay only into the channel $\pi^{-} e^{+} \nu$ while the $\left|\overline{\mathrm{K}}_{0}\right\rangle$

\footnotetext{
${ }^{25}$ For example, the damping corrections for the $n$th interference fringe of the experiment of Reference [49] where $p=229 \mathrm{MeV} / \mathrm{c}, \sigma_{p} / p=6.0 \times 10^{-7}$ (length of wavepacket $1 \mu \mathrm{m}$ ) and $r^{\prime}+\bar{r} \simeq 2 \mathrm{~m}$ are: $\Delta p /\left(2 \sigma_{p}\right)=n \bar{\gamma}^{2} h /\left[\sigma_{p}\left(r^{\prime}+\bar{r}\right)\right]=n 1.7 \times 10^{-9}$ and $\sigma_{p} \Delta r /(2 \hbar)=n \pi \sigma_{p} / p=n 1.9 \times 10^{-6}$.
} 
components decay only into $\pi^{+} e^{-} \nu$. The equations (9.26) and (9.27) then lead to the following relations between the semileptonic decay amplitudes:

$$
\left\langle\pi^{-} e^{+} \nu|T| \mathrm{K}_{\mathrm{S}}\right\rangle=\left\langle\pi^{-} e^{+} \nu|T| \mathrm{K}_{\mathrm{L}}\right\rangle=-\left\langle\pi^{+} e^{-} \nu|T| \mathrm{K}_{\mathrm{S}}\right\rangle=\left\langle\pi^{+} e^{-} \nu|T| \mathrm{K}_{\mathrm{L}}\right\rangle \equiv A_{e K}
$$

On the assumption that the $\mathrm{K}_{\mathrm{S}}, \mathrm{K}_{\mathrm{L}}$ mesons are produced at space-time points $x_{P S}, x_{P L}$ respectively, and decay at $x_{D}$, the probability amplitudes for the production of $e^{+}, e^{-}$via propagation of $\mathrm{K}_{\mathrm{S}}, \mathrm{K}_{\mathrm{L}}$ are given by (2.2) and (2.3) as:

$$
\begin{aligned}
& A_{D S}^{e^{+}}\left(\mathrm{K}_{\mathrm{S}}\right)=A_{e K} \int\left\langle D\left|\mathrm{~K}_{\mathrm{S}}\right| P S\right\rangle f\left(p_{S}\right) d p_{S} \\
& A_{D S}^{e^{+}}\left(\mathrm{K}_{\mathrm{L}}\right)=A_{e K} \int\left\langle D\left|\mathrm{~K}_{\mathrm{L}}\right| P S\right\rangle f\left(p_{L}\right) d p_{L} \\
& A_{D S}^{e^{-}}\left(\mathrm{K}_{\mathrm{S}}\right)=-A_{e K} \int\left\langle D\left|\mathrm{~K}_{\mathrm{S}}\right| P S\right\rangle f\left(p_{S}\right) d p_{S} \\
& A_{D S}^{e^{-}}\left(\mathrm{K}_{\mathrm{L}}\right)=A_{e K} \int\left\langle D\left|\mathrm{~K}_{\mathrm{L}}\right| P S\right\rangle f\left(p_{L}\right) d p_{L}
\end{aligned}
$$

The space-time propagators of the mesons are given by (3.12) as:

$$
\left\langle D\left|\mathrm{~K}_{\mathrm{i}}\right| P i\right\rangle=\frac{\beta_{i}}{L} \exp \left[-i \frac{\left[m_{i}-i \Gamma_{i} /\left(2 c^{2}\right)\right] c^{2} \Delta \tau_{i}}{\hbar}\right] \quad i=S, L
$$

The amplitudes $f\left(p_{S}\right), f\left(p_{L}\right)$ describe the momentum distribution of the mesons. As discussed below the dominant source of momentum smearing is radiative corrections rather than variation of the physical masses $W_{L}$ and $W_{S}$, of order $\Gamma_{L} / c^{2}, \Gamma_{S} / c^{2}$, of $\mathrm{K}_{\mathrm{L}}$ and $\mathrm{K}_{\mathrm{S}}$, due to their unstable nature. It is shown below that the precise form of $f(p)$ does not affect the final result for the detection probability. For convenience, $f(p)$ is normalised so that

$$
\int_{0}^{\infty} f(p)^{2} d p=1
$$

Since the decay widths are relatively large, indeed $\Gamma_{S}=2.1\left(m_{L}-m_{S}\right)$, the kinematical effects of the variation of the physical masses of the mesons are of a similar size to those, generated by the difference of pole masses, that underly the whole 'flavour oscillation' phenomenon and so cannot be, prima facie, neglected. This variation of the physical mass, $W$, modifies the exact expression, (9.14), for the propagator phase in the following way:

$$
\phi=-\frac{m c^{2} \tau}{\hbar}=-\frac{m c^{2} t}{\gamma \hbar}=-\frac{\left(m c^{2}\right)^{2} L}{E(W) v(W) \hbar}=-\frac{m c^{2} W L}{p \hbar}
$$

where

$$
E(W) \equiv \sqrt{\left(W c^{2}\right)^{2}+(p c)^{2}}, \quad v(W)=\frac{p c^{2}}{E(W)}
$$

The phase then depends, in general, on both the physical mass and the velocity (or momentum) of the particle. Unlike that of the photon in physical optics the particle velocity is variable, and unlike the electron in the YDSE discussed above, the velocity is not fixed by the value of the momentum.

In order to proceed further it is necessary to discuss the space-time structure of the production and detection events corresponding to the probability amplitudes. Only the 
spatial distance $L$ between the production and detection events is necessarily constant in the probability amplitudes (9.30)-(9.33). To give some feeling for the variation of the kinematical quantities and time intervals involved one may ask the following questions: (1) What is the difference of momentum necessary to compenstate the change in velocity due to the $\mathrm{K}_{\mathrm{S}}-\mathrm{K}_{\mathrm{L}}$ mass difference? (2) In case of equal momenta for the $\mathrm{K}_{\mathrm{S}}$ and $\mathrm{K}_{\mathrm{L}}$ what is the difference in their production times in order to arrive simultaneously at the detection event? If the velocities of the $\mathrm{K}_{\mathrm{S}}$ and $\mathrm{K}_{\mathrm{L}}$ are equal, their momenta $p_{L}$ and $p_{S}$ must satisfy the condition:

$$
\frac{v_{S}}{c}=\frac{1}{\sqrt{1+\left(\frac{W_{S} c}{p_{S}}\right)^{2}}}=\frac{v_{L}}{c}=\frac{1}{\sqrt{1+\left(\frac{W_{L} c}{p_{L}}\right)^{2}}}
$$

that is

$$
\frac{W_{S}}{W_{L}}=\frac{p_{S}}{p_{L}}
$$

so that

$$
\frac{\langle\delta p\rangle}{\langle p\rangle}=\frac{\left(\left\langle W_{L}\right\rangle-\left\langle W_{S}\right\rangle\right) c}{\langle p\rangle}=\frac{\left(m_{L}-m_{S}\right) c}{\langle p\rangle}=1.8 \times 10^{-14}
$$

where the value $\langle p\rangle=194 \mathrm{MeV} / \mathrm{c}$, corresponding to the centre-of-mass momentum of the $\mathrm{K}_{\mathrm{S}}$ and $\mathrm{K}_{\mathrm{L}}$ in the experiment [51], and $m_{L}-m_{S}=3.49 \times 10^{-12} \mathrm{Mev} / c^{2}$ have been used. This is many orders of magnitude smaller than than the momentum smearing of the $\mathrm{K}_{\mathrm{S}}$ and $\mathrm{K}_{\mathrm{L}}$ due to initial state photon radiation. This is estimated, using the soft photon radiatve correction formalism of [52] to be:

$$
\frac{\left\langle\delta p_{\text {rad }}\right\rangle}{\langle p\rangle}=4.2 \times 10^{-2}
$$

for the experiment [51]. In these circumstances the path amplitudes in (9.30)-(9.33) for different momenta and velocities, within the appropriate range given in (9.39), must contribute equally to the sum over intermediate states in (2.3), so that no damping of the interference term is to be expected from momentum or velocity variation. This is verified in the following calculation.

To answer the question (2), the difference of production times: $\Delta t_{S L} \equiv t_{P S}-t_{P L}$ for $\mathrm{K}_{\mathrm{S}}$ and $\mathrm{K}_{\mathrm{L}}$ of fixed momentum, in order to arrive simultaneously at the typical distance $c \beta \gamma \tau_{S}$ from their production point is calculated:

$$
\Delta t_{S L}=\frac{\left(m_{L}-m_{S}\right) \tau_{S}}{\bar{E}}
$$

Some values of $\Delta t_{S L}$ for different assumed values of $\bar{p}$ and $\bar{E}$ are presented in Table 2. Associating these with the lifetime of a hypothetical unstable source particle gives decay widths of such a particle in the range from 1 to $200 \mathrm{MeV}$. whereas the 'characteristic time' ${ }^{26}$ of the strong/weak interaction process that produces the $\Lambda \mathrm{K}_{\mathrm{S}}$ or $\Lambda \mathrm{K}_{\mathrm{L}}$ systems must be much shorter than this as no such resonant state is actually produced. In the following

\footnotetext{
${ }^{26}$ This is an interesting concept that has not been addressed, to date, by any physical theory. Presumably it takes some non-vanishing time for the quark rearrangement and creation processes that convert, in the present example, the $\pi^{-} p$ system into $\Lambda \mathrm{K}_{\mathrm{S}}$ or $\Lambda \mathrm{K}_{\mathrm{L}}$, to occur. The possibility to observe such a timeinterval in decay processes by measurable deviations from the exponential decay law or the Breit-Wigner line shape of an unstable particle has been suggested [53].
} 


\begin{tabular}{|c|ccccc|}
\hline $\bar{p}(\mathrm{GeV} / \mathrm{c})$ & 0.01 & 0.1 & 1.0 & 10.0 & 100.0 \\
$\bar{E}(\mathrm{GeV})$ & 0.498 & 0.508 & 1.117 & 10.1 & 100.0 \\
$\Delta t_{S L}(\mathrm{sec})$ & $6.27 \times 10^{-22}$ & $6.14 \times 10^{-22}$ & $2.8 \times 10^{-22}$ & $3.1 \times 10^{-23}$ & $3.1 \times 10^{-24}$ \\
\hline
\end{tabular}

Table 2: Momentum and energy dependence of $\Delta t_{S L}$, the difference in production times of $\mathrm{K}_{\mathrm{S}}$ and $\mathrm{K}_{\mathrm{L}}$ of equal momentum in order to arrive simultaneously at distance $c \beta \gamma \tau_{S}$ from their production point.

it is assumed that the 'characteristic time' is so short in comparison with the values of $\Delta t_{S L}$ shown in Table 2, that the production time of the $\mathrm{K}_{\mathrm{S}}$ and $\mathrm{K}_{\mathrm{L}}$ in the probability amplitudes (9.30)-(9.33) is the same in every case, i.e. $t_{P S}=t_{P L}=t_{P}$. Thus, since the detection event occurs some definite time, the $K_{S}$ and $K_{L}$ are assumed to have equal velocities in the alternative probability amplitudes. Note here the difference with the electron YDSE. There also the equal production time assumption is made, leading to the conventional de Broglie wavelength for the associated 'matter wave', but in this case due to the unique physical mass of the electron, and the different path lengths in the YDSE, different velocities are necessary.

Substituting (9.34) into (9.30)-(9.33) and using the expression for $\Delta \tau_{i}$ given by the second and last members of (9.36), it follows that:

$$
\begin{aligned}
\left|A_{D S}^{e^{+}}\left(\mathrm{K}_{\mathrm{S}, \mathrm{L}}\right)\right|^{2} & =\left|A_{D S}^{e^{-}}\left(\mathrm{K}_{\mathrm{S}, \mathrm{L}}\right)\right|^{2} \\
& =\left|\frac{A_{e K} \beta_{S, L} A_{P}}{L}\right|^{2} \int f\left(p_{S, L}^{\prime}\right) f\left(p_{S, L}\right) \delta\left(p_{S, L}-\left(p_{S, L}^{\prime}\right) d p_{S, L}^{\prime} d p_{S, L}\right. \\
& \simeq\left|\frac{A_{e K} \bar{\beta} A_{P}}{L}\right|^{2} \exp \left[-\frac{\Gamma_{S, L} W_{S, L} m_{S, L} L}{\langle p\rangle \hbar}\right] \\
& \equiv\left|A_{e p}\right|^{2} \exp \left[-\frac{\Gamma_{S, L} W_{S, L} m_{S, L} L}{\langle p\rangle \hbar}\right]
\end{aligned}
$$

where the approximations $\beta_{L} \simeq \beta_{S}=\bar{\beta}$ and $W_{L} \simeq W_{S}=\bar{m} \equiv\left(m_{L}+m_{S}\right) / 2$ have been made and $\langle p\rangle$ denotes the average value of $p_{L}$ or $p_{S}$.

Replacing the path labels A and B in $\operatorname{Eqn}(9.2)$ by $S$ and $L$ respectively, the interference terms $I_{S L}^{e^{ \pm}}$for the detection of $e^{ \pm}$are given by (9.30)-(9.36) as :

$$
\begin{aligned}
I_{S L}^{e^{ \pm}}= & \pm 2 R e\left|A_{e p}\right|^{2} \iint \exp \left\{-\frac{1}{2 \hbar}\left[\frac{\Gamma_{S} W_{S}}{p_{S}}+\frac{\Gamma_{L} W_{L}}{p_{L}}\right]\right\} \exp \left\{\frac{i c^{2}}{\hbar}\left[\frac{m_{S} W_{S}}{p_{S}}-\frac{m_{L} W_{L}}{p_{L}}\right] L\right\} \\
& \times f\left(p_{S}\right) f\left(p_{L}\right) \delta\left(W_{S} p_{L}-W_{L} p_{S}\right) d p_{L} d p_{S}
\end{aligned}
$$

where the $\delta$-function imposes the equal velocity condition (9.38) and the approximation $\beta_{S} \beta_{L} \simeq \bar{\beta}^{2}$ has been made. Performing the $p_{S}$ integral in (9.43) gives:

$$
\begin{aligned}
I_{S L}^{e^{ \pm}}= & \pm 2 R e\left|A_{e p}\right|^{2} \int \exp \left\{-\frac{\left(\Gamma_{S}+\Gamma_{L}\right)}{2 \hbar} \frac{W_{L} L}{p_{L}}\right\} \exp \left\{\frac{i c^{2} \Delta m_{L S}}{\hbar} \frac{W_{L}}{p_{L}} L\right\} \\
& \times f\left(\frac{W_{S} p_{L}}{W_{L}}\right) f\left(p_{L}\right) d p_{L}
\end{aligned}
$$

where $\Delta m_{L S} \equiv m_{L}-m_{S}$. Since the ratio $W_{L} / W_{S}$ differs from unity only by quantities of order $\Gamma_{S} / c^{2} \bar{m} \simeq\left(3.49 \times 10^{-12} \mathrm{MeV}\right) /(498 \mathrm{MeV})=7.0 \times 10^{-15}$, then, to a very good 
approximation, the replacements: $W_{L} / W_{S}=1, W_{L}=\bar{m}$ may be made in (9.44) giving:

$$
I_{S L}^{e^{ \pm}}= \pm 2 R e\left|A_{e p}\right|^{2} \exp \left\{-\frac{\left(\Gamma_{S}+\Gamma_{L}\right)}{2 \hbar} \frac{\bar{m} L}{\langle p\rangle}\right\} \cos \left(\frac{\bar{m} c^{2} \Delta m_{L S} L}{\hbar\langle p\rangle}\right)
$$

where the normalisation condition (9.35) has been used.

Subsitituting (9.42) and (9.45) into (9.2) gives finally for the probability, $P\left(e^{ \pm}, L,\langle p\rangle\right)$ to detect $e^{ \pm}$at distance $L$ from the production point of $\mathrm{K}_{\mathrm{S}}, \mathrm{K}_{\mathrm{L}}$ with momentum $\langle p\rangle$ :

$$
\begin{aligned}
P\left(e^{ \pm}, L,\langle p\rangle\right)= & \left|A_{e p}\right|^{2}\left\{\exp \left[-\frac{\Gamma_{S} \bar{m} c^{2} L}{\hbar\langle p\rangle}\right]+\exp \left[-\frac{\Gamma_{L} \bar{m} c^{2} L}{\hbar\langle p\rangle}\right]\right. \\
& \left. \pm 2 \exp \left[-\frac{\left(\Gamma_{S}+\Gamma_{L}\right)}{2 \hbar} \frac{\bar{m} c^{2} L}{\langle p\rangle}\right] \cos \left(\frac{\bar{m} c^{2} \Delta m_{L S} L}{\hbar\langle p\rangle}\right)\right\}
\end{aligned}
$$

On the assumption that the $\mathrm{K}_{\mathrm{S}}$ and $\mathrm{K}_{\mathrm{L}}$ mesons have the same velocity this probability may be expressed in terms of the proper time interval $\tau$ between their production and decay via the relation (see (9.36):

$$
\tau=\frac{W L}{\langle p\rangle} \simeq \frac{\bar{m} L}{\langle p\rangle}
$$

so that

$$
\begin{aligned}
P\left(e^{ \pm}, \tau\right)= & \left|A_{e P}\right|^{2}\left\{\exp \left[-\frac{\Gamma_{S} \tau}{\hbar}\right]+\exp \left[-\frac{\Gamma_{L} \tau}{\hbar}\right]\right. \\
& \left. \pm 2 \exp \left[-\frac{\left(\Gamma_{S}+\Gamma_{L}\right) \tau}{2 \hbar}\right] \cos \left(\frac{\Delta m_{L S} c^{2} \tau}{\hbar}\right)\right\}
\end{aligned}
$$

This is just the formula that has been used till now to interpret such quark flavour oscillation experiments $[51,54,55]$. Thus the Feynman path amplitude calculation, where careful account is taken of all relevant physical parameters, particularly with respect to the space-time structure of the production and detection events, gives the same result as a simple calculation of the phase difference $\phi_{S}-\phi_{L}$, neglecting all such considerations, using the time-dependent Schrödinger equation in the respective rest frames of the $\mathrm{K}_{\mathrm{S}}$ and $\mathrm{K}_{\mathrm{L}}$ mesons..

\section{Neutrino Oscillations}

Previous discussions of neutrino oscillations using the Feynman path amplitude formalism can be found in $[11,12,13]$. The experiment to be discussed here is detection of the process: $\nu n \rightarrow e^{-} p$, where the incoming neutrino is produced by pion decay at rest: $\pi^{+} \rightarrow \mu^{+} \nu$. The result found generalises in a straightforward manner to any neutrino oscillation experiment where the neutrino source is at rest. The experiment is shown in more detail in Fig.11. A slow $\pi^{+}$comes to rest at time $t_{0}$ in a stopping target $\mathrm{T}$ (Fig.11 a)). For simplicity, the case of only two neutrino flavours is considered. The neutrino mass eigenstates $\left|\nu_{1}\right\rangle$ and $\left|\nu_{2}\right\rangle$ have pole masses $m_{1}$ and $m_{2}$ where $m_{1}>m_{2}{ }^{27}$. Fig.11

\footnotetext{
${ }^{27}$ Note that the subscripts ' 1 ' and '2' are arbitary, and do not necessarily correspond to fermion generation number. In the presently favoured interpretation of atmospheric neutrino oscillations [58], where there is an important contribution from pion decay neutrinos, the mass eigenstates are those associated with the second and third generations
} 
b) and c) show two alternative histories for the stopped pion. In Fig11 b), the decay $\pi^{+} \rightarrow \mu^{+} \nu_{1}$ occurs at time $t_{1}$, in Fig.12 c) the decay $\pi^{+} \rightarrow \mu^{+} \nu_{2}$ occurs at a later time $t_{2}$. With a suitable time difference $t_{2}-t_{1}$, the neutrinos corresponding to the two alternative paths arrive at the detection event at the same time, $t_{D}$ and are thus indistinguishable (Fig.11 d)). The corresponding probability amplitudes $A_{e \mu}^{(1)}, A_{e \mu}^{(2)}$ then add, as in (9.1), to give the total probability amplitude for the experiment. The labels 'A' and ' $\mathrm{B}$ ' in (9.1) and (9.2) are here replaced by '(1)' and '(2)' corresponding to the mass eigenstates $\nu_{1}$ and $\nu_{2}$.

Note that the physical situation is strictly analagous to that of the Michelson Interferometer or the photon YDSE. In these experiments the photon must be emitted at different times by the source, in the interfering amplitudes, in order to arrive in time coincidence at the detection event. In these two experiments the time difference is necessary to compensate the non-equal path lengths. In a neutrino oscillation experiment, both paths have the same length, and the time difference of neutrino emission is necessary to compensate for the different neutrino velocities.

Introducing the process amplitudes for the $\pi$-decay and neutrino detection processes, and the space-time propagators of the pion source and the neutrinos according to (3.12), the probability amplitudes for propagation of the states $\left|\nu_{1}\right\rangle$ and $\left|\nu_{2}\right\rangle$ are:

$$
A_{e \mu}^{(i)}=\left\langle e^{-} p|T| \nu n\right\rangle U_{e i}\left\langle D\left|\nu_{i}\right| i\right\rangle U_{\mu i}\left\langle\nu \mu^{+}|T| \pi^{+}\right\rangle\left\langle i\left|\pi^{+}\right| 0\right\rangle \quad(i=1,2)
$$

the space-time propagators of the source pion (assumed to be at rest so that $\Delta \tau=\Delta t$ ) and the neutrinos are:

$$
\begin{aligned}
\left\langle i\left|\pi^{+}\right| 0\right\rangle & =\exp \left[-i \frac{\left[m_{\pi}-i \Gamma_{\pi} /\left(2 c^{2}\right)\right] c^{2}\left(t_{i}-t_{0}\right)}{\hbar}\right] \quad(i=1,2) \\
\left\langle D\left|\nu_{i}\right| i\right\rangle & =\exp \left[-i \frac{m_{i} c^{2}\left(\tau_{D}-\tau_{i}\right)}{\hbar}\right] \quad(i=1,2)
\end{aligned}
$$

The process amplitudes for pion decay and neutrino detection are written as products of 'reduced' process amplitudes and elements of the Maki-Nakagawa-Sakata [57] matrix $U_{\ell i}$ that describes the strength of the charged-current coupling between lepton $\ell$ and the neutrino mass eigenstate $\left|\nu_{i}\right\rangle$ :

$$
\begin{aligned}
\left\langle e^{-} p|T| \nu_{i} n\right\rangle & =U_{e i}\left\langle e^{-} p|T| \nu n\right\rangle \quad(i=1,2) \\
\left\langle\nu_{i} \mu^{+}|T| \pi^{+}\right\rangle & =U_{\mu i}\left\langle\nu \mu^{+}|T| \pi^{+}\right\rangle \quad(i=1,2)
\end{aligned}
$$

In the case of only two charged lepton and neutrino flavours, all the elements of the MNS matrix are real and are specified by a single mixing angle $\theta_{12}$ :

$$
U_{e 1}=U_{e 2}=\cos \theta_{12}, \quad U_{e 2}=-U_{\mu 1}=\sin \theta_{12}
$$

Denoting the phase of $A_{e \mu}^{(i)}$ as $\phi^{(i)},(9.49)-(9.51)$ give:

$$
-\frac{\hbar \phi^{(i)}}{c^{2}}=m_{i}\left(\tau_{D}-\tau_{i}\right)+m_{\pi}\left(t_{i}-t_{0}\right) \quad(i=1,2)
$$



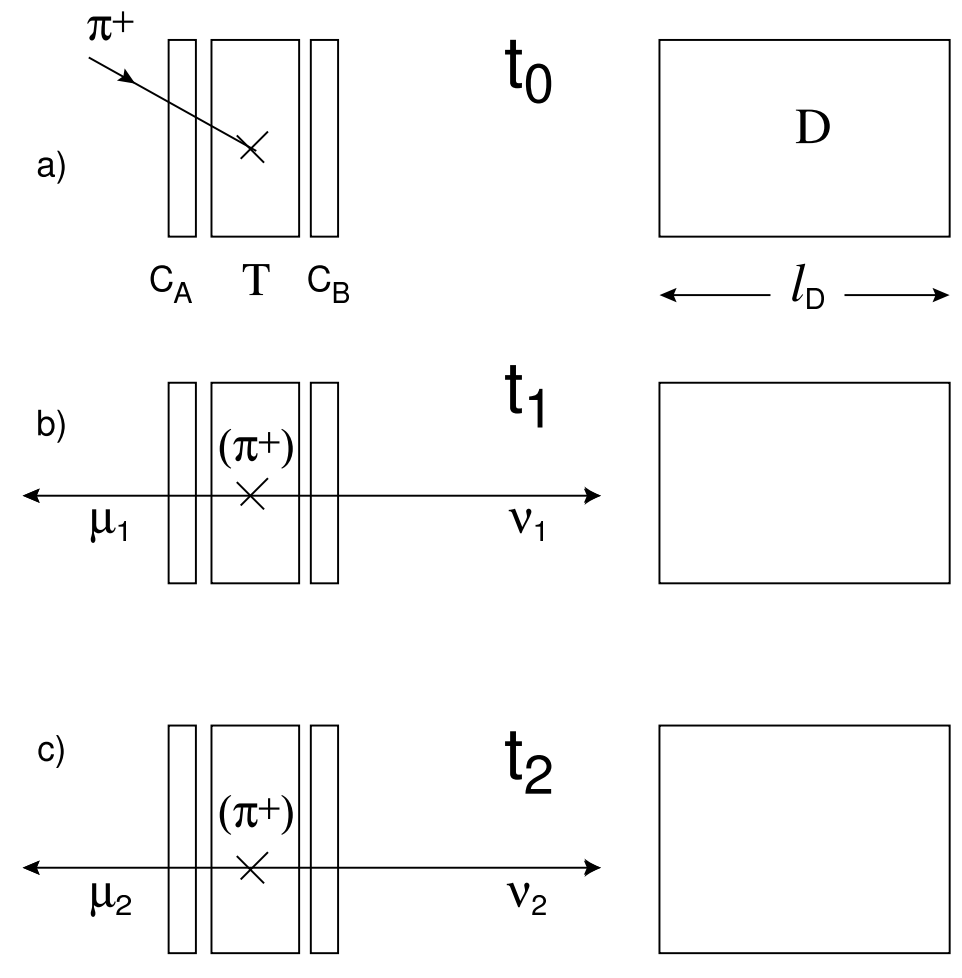

d)

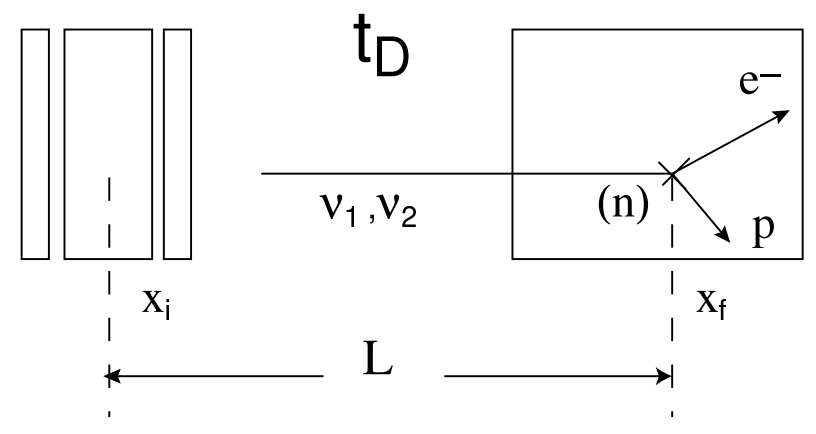

Figure 11: An experiment to measure neutrino oscillations following pion decay. a) a $\pi^{+}$ comes to rest in a stopping target $T$ at time $t_{0}$. b) and $c$ ) show alternative histories of the stopped pion. In $b$ ) the decay $\pi^{+} \rightarrow \mu \nu_{1}$ occurs at time $t_{1}$. In c) the decay $\pi^{+} \rightarrow \mu \nu_{2}$ occurs at time $t_{2}$. If $m_{1}>m_{2}$, and with a suitable value of $t_{2}-t_{1}$, the neutrinos may arrive at the same time $t_{D}$ in the alternative histories at the the detection event, as shown in d). Since the detection event $\left(\nu_{1}, \nu_{2}\right) n \rightarrow p e^{-}$does not distinguish the neutrino flavour, the amplitudes corresponding to the different histories in b) and c) must be added, as in (9.1), to give the probability amplitude for the experiment. Note the similarity of the sequence of space-time events to that in the Michelson interferometer of Fig.8. 
Using the last member of (9.14) to re-write the first term on the RHS of (9.55) and noting that:

$$
t_{i}-t_{0}=t_{D}-t_{0}-\frac{L}{v_{i}} \quad(i=1,2)
$$

gives

$$
\begin{aligned}
-\frac{\hbar \phi^{(i)}}{c^{2}} & =\frac{m_{i}^{2} L}{p_{i}}+m_{\pi}\left(t_{D}-t_{0}\right)-\frac{m_{\pi} L}{v_{i}} \\
& =\frac{m_{i}^{2} L}{p_{0}}\left[1-\frac{c m_{\pi}}{2 p_{0}}\right]+m_{\pi}\left(t_{D}-t_{0}-\frac{L}{c}\right)+O\left[m_{i}^{4}\right] \quad(i=1,2)
\end{aligned}
$$

where the neutrinos are assumed to be ultra-relativistic so that:

$$
v_{i}=c\left(1-\frac{c^{2} m_{i}^{2}}{2 p_{0}^{2}}\right)+O\left[m_{i}^{4}\right] \quad(i=1,2)
$$

In (9.57) and (9.58) $p_{0}$ is the centre-of-mass momentum of a massless neutrino produced in the decay $\pi \rightarrow \mu \nu$

$$
\frac{p_{0}}{c}=\frac{m_{\pi}^{2}-m_{\mu}^{2}}{2 m_{\pi}}=29.8 \mathrm{MeV} / \mathrm{c}
$$

The replacements $A_{F I}^{A, B} \rightarrow A_{e \mu}^{(1),(2)}$ in (9.2) give then, for the probability of electron detection at distance $L$ from the neutrino source:

$$
\begin{aligned}
P_{e \mu}= & \int_{t_{m i n}}^{\infty} d t_{D}\left|\frac{\left\langle e^{-} p|T| \nu n\right\rangle\left\langle\nu \mu^{+}|T| \pi^{+}\right\rangle}{L}\right|^{2} \exp \left[-\frac{\Gamma_{\pi}\left(t_{D}-t_{0}\right)}{\hbar}\right] \\
& \times\left\{\left|U_{e 1} U_{\mu 1}\right|^{2} \exp \left[\frac{\Gamma_{\pi} t_{1}^{f l}}{\hbar}\right]+\left|U_{e 2} U_{\mu 2}\right|^{2} \exp \left[\frac{\Gamma_{\pi} t_{2}^{f l}}{\hbar}\right]\right. \\
& \left.+2 U_{e 1} U_{e 2} U_{\mu 1} U_{\mu 2} \exp \left[\frac{\Gamma_{\pi}\left(t_{1}^{f l}+t_{2}^{f l}\right)}{2 \hbar}\right] \cos \left(\frac{\Delta\left(m_{12}\right)^{2} c^{2}}{p_{0}}\left[\frac{c m_{\pi}}{2 p_{0}}-1\right] \frac{L}{\hbar}\right)\right\}(9
\end{aligned}
$$

where

$$
\Delta\left(m_{12}\right)^{2} \equiv m_{1}^{2}-m_{2}^{2}
$$

In (9.60) the neutrino times-of-flight, $t_{i}^{f l}$, have been introduced:

$$
t_{i}^{f l} \equiv t_{D}-t_{i}=\frac{L}{v_{i}}=\frac{L}{c}\left(1+\frac{c^{2} m_{i}^{2}}{2 p_{0}^{2}}\right)+O\left[m_{i}^{4}\right] \quad(i=1,2)
$$

and the unobserved detection time $t_{D}$ has been integrated out. Consideration of Fig.12 shows that the values of $t_{\min }$ are: $t_{0}+t_{1}^{f l}$ for the propagation of neutrinos of mass $m_{1}$ only, $t_{0}+t_{2}^{f l}$ for the propagation of neutrinos of mass $m_{2}$ only, and $t_{0}+t_{1}^{f l}$ for the interference term in (9.60). This is because both neutrino paths must be possible if interference is to occur, and $t_{1}^{f l}>t_{2}^{f l}$. Performing the $t_{D}$ integrations in (9.60) and substituting the values of the MNS matrix elements from (9.54) then gives the final result:

$$
\begin{aligned}
P_{e \mu}= & \frac{2 \hbar}{\Gamma_{\pi}}\left|\frac{\left\langle e^{-} p|T| \nu n\right\rangle\left\langle\nu \mu^{+}|T| \pi^{+}\right\rangle}{L}\right|^{2} \sin ^{2} \theta_{12} \cos ^{2} \theta_{12} \\
& \times\left\{1-\exp \left[-\frac{\Gamma_{\pi} c \Delta\left(m_{12}\right)^{2} L}{4 \hbar p_{0}^{2}}\right] \cos \left(\frac{\Delta\left(m_{12}\right)^{2} c^{2}}{p_{0}}\left[\frac{c m_{\pi}}{2 p_{0}}-1\right] \frac{L}{\hbar}\right)\right\}
\end{aligned}
$$


The exponential damping correction of the interference term due to the non-vanishing pion lifetime is completely neligible ${ }^{28}$

The prediction (9.61) is readily generalised to the case of an arbitary two-flavour neutrino oscillation experiment with a stationary source, $S$ :

$$
S \rightarrow X_{S}+\bar{\ell}_{j}+\nu_{i}, \quad \nu_{i}+T_{D} \rightarrow X_{D} \ell_{k}
$$

to yield the prediction:

$$
\begin{aligned}
P_{k j}= & \frac{2 \hbar}{\Gamma_{S}}\left|\frac{\left\langle\ell_{k} X_{D}|T| \nu T_{D}\right\rangle\left\langle\nu \bar{\ell}_{j} X_{S}|T| S\right\rangle}{L}\right|^{2} \times \\
& \left\{\left|U_{k 1} U_{j 1}\right|^{2}+\left|U_{k 2} U_{j 2}\right|^{2}\right. \\
& \left.+2 U_{k 1} U_{k 2} U_{j 1} U_{j 2} \cos \left[\frac{\Delta\left(m_{12}\right)^{2}}{m_{S}}\left(\frac{R_{m}}{1-R_{m}^{2}}\right)^{2} \frac{L}{\hbar}\right]\right\}
\end{aligned}
$$

Here $\ell_{i}\left(\bar{\ell}_{i}\right)$ denotes a charged lepton (antilepton) of fermion generation $i$ and

$$
R_{m} \equiv \frac{m_{R}}{m_{S}}, \quad\left(c m_{R}\right)^{2} \equiv\left(p\left(X_{S}\right)+p\left(\bar{\ell}_{j}\right)\right)^{2}
$$

Here $p\left(X_{S}\right)$ and $p\left(\bar{\ell}_{j}\right)$ are 4 -vectors, $m_{S}$ is the mass of the source particle and $m_{R}$ is the effective mass of the particle, or particle system, $X_{S}+\bar{\ell}_{j}$, recoiling from the neutrino mass eigenstate. For the case of $\beta$-decay, where:

$$
E_{\beta}=E\left(\nu_{i}\right)+E\left(\bar{\ell}_{j}\right)=\text { constant }
$$

the argument $\phi^{(1)}-\phi^{(2)}$ of the cosine in (9.62) is replaced by $[11,12]$ :

$$
\phi^{(1)}-\phi^{(2)}=\frac{\Delta\left(m_{12}\right)^{2}}{p_{\nu}}\left[\frac{E_{\beta}}{2 p_{\nu}}-1\right]
$$

It is instructive to now compare in more detail the neutral kaon oscillation and neutrino oscillation cases. In Table 2 is shown the difference in production times of $K_{S}$ and $\mathrm{K}_{\mathrm{L}}$ mesons of various fixed momenta in order that they arrive simultaneously, in the alternative histories corresponding to either $\mathrm{K}_{\mathrm{S}}$ or $\mathrm{K}_{\mathrm{L}}$ propagation, at a detection event at a typical decay distance $c \beta \gamma \tau_{S}$. A similar comparison is now made for neutrino oscillations by considering the difference of production time $\Delta t_{12}=t_{2}-t_{1}$ in order that the neutrinos $\nu_{1}$ and $\nu_{2}$ arrive simultaneously at the detection event, as shown in Fig.11 d). The distance, $L(\pi)$, between the production and detection events is chosen so that the phase of the cosine term in (9.62) is $\pi \mathrm{rad}$. Thus:

$$
L(\pi)=\frac{h p_{0}}{\Delta\left(m_{12}\right)^{2} c^{2}\left(\frac{c m_{\pi}}{p_{0}}-2\right)}=\frac{13.8 \mathrm{~m}}{\left.\Delta\left(m_{12}\right)^{2} c^{4}(\mathrm{eV})^{2}\right)}
$$

\footnotetext{
${ }^{28}$ Setting $\Delta\left(m_{12}\right)^{2} c^{2} L /\left(p_{0} \hbar\right)$ to unity, so that the argument of the cosine in (9.62) is of the same order, as necessary for an observable neutrino oscillation effect, gives for the damping term: $\exp \left[-\Gamma_{\pi} /\left(4 p_{0}\right)\right]=$ $\exp \left[-4.0 \times 10^{-16}\right]$.
} 
The corresponding production time difference is:

$$
\Delta t_{21}=L(\pi)\left(\frac{1}{v_{1}}-\frac{1}{v_{2}}\right)=\frac{L(\pi)}{c} \frac{\Delta\left(m_{12}\right)^{2} c^{2}}{2 p_{0}^{2}}+O\left[m_{i}^{4}\right]
$$

where (9.57) has been used. Combining (9.65) and (9.66) gives:

$$
\Delta t_{21}=\frac{h}{4 c\left(\frac{m_{\pi} c}{2}-p_{0}\right)}=8.22 \times 10^{-24} \mathrm{sec}
$$

Thus $\Delta t_{21}$ depends only on the kinematics of the neutrino production process and is independent of the neutrino mass difference. For neutrinos produced in pion decay, $\Delta t_{21}$ is a factor of $3 \times 10^{-16}$ smaller than the pion mean lifetime of $2.6 \times 10^{-8} \mathrm{sec}$. This implies strictly equal amplitudes for the decay processes shown in Fig.11 b) and c) with completely negligible lifetime damping effects as discussed above. Further estimates of the latter may be found in [12]. The situation is thus dramatically different from the neutral kaon flavour oscillation case where, instead, the time differences for simultaneous arrival of equal momentum $\mathrm{K}_{\mathrm{S}}$ and $\mathrm{K}_{\mathrm{L}}$ mesons at the detection event shown in Table 2 are much larger than the characteristic time of the production process. Thus the $\mathrm{K}_{\mathrm{S}}$ and $\mathrm{K}_{\mathrm{L}}$ are produced at essentially the same time. Because of the tiny $\mathrm{K}_{\mathrm{S}}-\mathrm{K}_{\mathrm{L}}$ mass difference:

$$
\Delta m_{12} / \bar{m}=3.49 \times 10^{-12} \mathrm{MeV} / \mathrm{c}^{2} / 497.7 \mathrm{MeV} / \mathrm{c}^{2}=7.0 \times 10^{-15}
$$

momentum and velocity smearing effects due to variation of the physical masses of the $\mathrm{K}_{\mathrm{S}}$ and $\mathrm{K}_{\mathrm{L}}$ and radiative corrections ensure that the probability amplitudes for $\mathrm{K}_{\mathrm{S}}$ and $\mathrm{K}_{\mathrm{L}}$ of the same velocity are closely equal, leading to the conventionally used formula (9.47). In the neutrino case however inspection of equal velocity condition analgous to (9.38):

$$
\frac{m_{1}}{m_{2}}=\frac{p_{1}}{p_{2}}
$$

shows that it may be physically impossible for $\nu_{1}$ and $\nu_{2}$ to have the same velocity in the interfering probability amplitudes.

The current analysis of atmospheric neutrino oscillations where pion decays give an important contribution estimates the mass difference to be [58]:

$$
\Delta\left(m_{12}\right)^{2} c^{4} \simeq 2 \times 10^{-3}(\mathrm{eV})^{2}
$$

In order to respect the equal velocity condition (9.68) the smearing, $\delta p$, of the neutrino momentum must be such that:

$$
\frac{\delta p}{\bar{p}}=\frac{\Delta m_{12}}{\bar{m}}=\frac{m_{1}-m_{2}}{\bar{m}}
$$

Now,

$$
\Delta\left(m_{12}\right)^{2}=\left(m_{1}-m_{2}\right)\left(m_{1}+m_{2}\right)=2 \Delta m_{12} \bar{m}
$$

So, combining (9.70) and (9.71), equal velocities require that:

$$
\frac{\delta p}{\bar{p}}=\frac{\Delta\left(m_{12}\right)^{2}}{2(\bar{m})^{2}}
$$


Assuming $\bar{m}=0.5 \mathrm{eV} / c^{2}$, which is consistent with all direct upper limits on neutrino masses [56], and inserting the experimental value of $\Delta\left(m_{12}\right)^{2}$ from (9.69) gives:

$$
\frac{\delta p}{\bar{p}}=4 \times 10^{-3}
$$

The dominant source of momentum smearing in pion decay is radiation of real photons. Using the soft photon formalism of [52] to calculate the mean momentum smearing, $\left\langle\delta p_{\text {rad }}\right\rangle$ due to soft photon emission gives:

$$
\frac{\left\langle\delta p_{\text {rad }}\right\rangle}{\langle p\rangle}=3.5 \times 10^{-4}
$$

which is incompatible with the equal velocity condition (9.73). Indeed the latter condition can be consistent with (9.74) only if $\bar{m}$ is close to the current direct upper limit on $m_{\nu_{1}}$ of $3 \mathrm{eV} / c^{2}[56]$. In this case $\delta p / \bar{p} \simeq 1.0 \times 10^{-4}$, which is just compatible with (9.74). There is still, however, no physical reason why probability amplitudes with equal velocities and production times should be favoured over those with different velocities and different production times.

The difficulty to justify the equal velocity hypothesis for neutrinos in view of the condition (9.68) has been previously pointed out in the literature [59,60] but the authors of these papers still concluded that the 'standard' formula for the neutrino oscillation phase [58], which will now be discussed, was correct.

As pointed out in [11], the derivation of the standard formula requires that the neutrino velocities defined kinematically according to the formula $v_{k i n} \equiv p c^{2} / E$ are different for different mass eigenstates, but that the space-time velocities: $v_{s-t} \equiv L / t$ are the same. These manifestly contradictory hypotheses (if neutrinos are indeed real (on-shell) particules propagating in space-time) give, instead of the prediction of (9.63) for the oscillation phase:

$$
\phi_{12}=\phi^{(1)}-\phi^{(2)}=\frac{\Delta\left(m_{12}\right)^{2} c}{m_{S} \hbar}\left(\frac{R_{m}}{1-R_{m}^{2}}\right)^{2} L+O\left[m_{i}^{4}\right]
$$

the standard formula:

$$
\phi_{12}^{\text {stand }}=\phi^{(1)}-\phi^{(2)}=\frac{\Delta\left(m_{12}\right)^{2} c^{2}}{2 p_{0} \hbar} L+O\left[m_{i}^{4}\right]
$$

This is actually the same result as found here for the neutral kaon oscillation phase (9.45) since:

$$
\bar{m} \Delta m_{12}=\frac{\left(m_{1}+m_{2}\right)}{2}\left(m_{1}-m_{2}\right)=\frac{\Delta\left(m_{12}^{2}\right)}{2}
$$

A simple derivation of (9.76) can be found in [58]. Each neutrino mass eigenstate is assumed to evolve temporally in its rest frame according to the time-dependent Schrödinger Equation. The corresponding phase increment is correctly given by the first member of (9.14) above. The Lorentz invariance of the phase is then used to write it in terms of laboratory-frame quantities:

$$
\phi^{(i)}=-i \frac{m_{i} c^{2} \tau_{i}}{\hbar} \simeq-i \frac{\left(E_{i} t-p_{i} c L\right)}{\hbar} \quad(i=1,2)
$$


It can be seen that it is just at this point in the derivation, that the assumption of equal propagation times, $t$, for each mass eigenstate implies equal space-time velocities $L / t$ whereas the kinematical velocities $p_{i} c^{2} / E_{i}$ are assumed to be different. As discussed in detail in [11] it is this unphysical assumption in the approximation made in the last member of (9.77) that leads to the factor of 2 difference between (9.76) and the neutrino propagation phase given by the exact relation (9.14). The standard formula (9.76) is derived from $\phi^{(1)}-\phi^{(2)}$, as given by $(9.77)$, on making the further assumptions that the neutrinos are ultra-relativistic, that $L / t=c$, and retaining only the leading $\Delta\left(m_{12}\right)^{2}$ neutrino mass terms. Evidently, since (9.77) assumes equal production times for different mass eigenstates, there is no contribution to the oscillation phase from the source particle in the standard formula.

The standard formula (9.76) is still universally employed by the neutrino physics community. Claims that the Feynman path amplitude formula (9.75) is incorrect have been made [61] and rebutted [11,62]. A critical discussion of the ad hoc 'wavepacket' approach, that is frequently used in discussions in the literature of the quantum mechanics of neutrino oscillations, that results, - from the point-of-view of Feynman's formulation of quantum mechanics embodied in the laws I-V presented in Section 2 - an artificial and unphysical blurring of the space-time structure of production and detection events, can be found in $[11,12]$.

The formula for the production time difference, (9.66) becomes, in the general case described by (9.67):

$$
\Delta t_{21}=\frac{h}{2 m_{S} c^{2} R_{m}^{2}}
$$

Thus, when the mass of the system recoiling against the neutrino becomes very small in comparison with the mass of the source particle, $\Delta t_{21}$ becomes very large, as does also the 'oscillation length, $L_{o s c}$, defined by writing the argument of the cosine interference term in (9.63) as $2 \pi L / L_{\text {osc }}$ so that:

$$
L_{o s c} \equiv \frac{m_{S} h}{\Delta\left(m_{12}\right)^{2} c}\left(\frac{1-R_{m}^{2}}{R_{m}}\right)^{2}
$$

This dependence of the oscillation length on the kinematics of the neutrino production process gives a straightforward experimental method to discriminate between the path amplitude prediction for the oscillation length, (9.79) and that predicted by the standard formula (9.76) which is:

$$
L_{\text {osc }}^{\text {stand }} \equiv \frac{2 p_{0} h}{\Delta\left(m_{12}\right)^{2} c^{2}}
$$

As proposed in [13], if a terrestrial long linebase ' $\nu_{\mu}$ disappearence' experiment such as $\mathrm{K} 2 \mathrm{~K}$ [63] is performed using neutrinos produced in the process: $\mathrm{K} \rightarrow \mu \nu$, rather than $\pi \rightarrow \mu \nu$ as in the existing experiment, the path amplitude formula predicts that the oscillation length is a factor of $\simeq 28$ times longer for a kaon source than for a pion one yielding the same neutrino momentum. This implies a strong suppression of the ' $\nu_{\mu}$ disappearence' phenomenon when using a kaon rather than a pion source. The standard formula predicts the same oscillation length for pion and kaon sources giving neutrinos of the same momentum. 


\begin{tabular}{|c||c|c|c|c|}
\hline Experiment & Photon YDSE & Electron YDSE & $\mathrm{K}_{0}$ Osc. & $\nu$ Osc. \\
\hline \hline$\delta r=0 ?$ & No & No & Yes & Yes \\
$\delta t=0 ?$ & No & Yes & Yes & No \\
$\delta v=0 ?$ & Yes & No & Yes & No \\
$\delta \phi^{\text {source }}=0 ?$ & No & Yes & Yes & No \\
$\delta \phi^{\text {part }}=0 ?$ & Yes & No & No & No \\
$\hbar\left(\phi_{B}-\phi_{A}\right)$ & $-\bar{p} \Delta r$ & $-\bar{p} \Delta r$ & $-\frac{\Delta\left(m_{L S}\right)^{2} c^{2}}{2 \bar{p}} L$ & $-\frac{\Delta\left(m_{12}\right)^{2} c}{m_{S}}\left(\frac{R_{m}}{1-R_{m}^{2}}\right)^{2} L$ \\
$\lambda^{\text {eff }} / \lambda^{D B}$ & 1 & 1 & $2\left(\frac{\bar{p}}{m_{S} c}\right)^{2}$ & $\frac{\bar{p}}{m_{S} c}\left(\frac{1-R_{m}^{2}}{R_{m}}\right)^{2}$ \\
\hline
\end{tabular}

Table 3: Comparison of the four 'two probabilty amplitude' experiments. The characteristics of each experiment are specified by the quantities: $\delta r, \delta t, \delta v, \delta \phi^{\text {source }}$ and $\delta \phi^{\text {part }}$ which are the diffrences of; path length, time-of-flight, velocity, source phase and particle phase for the two interfering amplitudes. Also shown is the interference phase $\phi_{B}-\phi_{A}$ in (9.2) and the ratio of the effective wavelength defined in (9.80) to the de Broglie wavelength.

The four different 'two probability amplitude' experiments that have just been discussed are summarised in Table 3. Each experiment is first classified in terms of its space-time properties. These are specified by the differences between the path lengths $(\delta r)$, the times-of-flight $(\delta t)$ and the particle velocities $(\delta v)$ for the two paths. This information is given as a Yes/No answer to the questions: $\delta r=0 ?, \delta t=0 ?$ and $\delta v=0$ ? Similarly the contributions to the interference phase from the source and the propagating particles are specified by the differences $\delta \phi^{\text {source }}$ and $\delta \phi^{\text {part }}$ for the two paths and given as answers to the questions $\delta \phi^{\text {source }}=0$ ? and $\delta \phi^{\text {part }}=0$ ?. In the last two rows the values of the interference phase and the ratio $\lambda^{e f f} / \lambda^{D B}$ are reported. The effective wavelength, $\lambda^{e f f}$ is defined for neutral kaon abd neutrino oscillations in terms of the phase difference $\Delta \phi$ for paths of length $L$ by the relation:

$$
\lambda^{e f f} \equiv \frac{2 \pi L}{|\Delta \phi|}
$$

while $\lambda^{D B}$ is the conventional de Broglie wavelength of the propagating particle as given by $(9.19)$.

The puzzling and difficult-to-understand concept of 'wave-particle duality' does not survive the detailed analysis of the superficially analogous, but in fact very different, experiments presented in Table 3. The 'wave' associated with the particle is different in every case. Although both the YDSEs may be simply (and correctly) interpreted in terms of the de Broglie wavelengths of the photon or the electron, the physical origin of the 'wave' is quite different in the two cases. For the photon it is the phase of the propagator of the excited source atom; for the electron it is the phase of the propagator of the latter. The effective wavelength for neutral kaons, although originating entirely from their propagators is quite different from that of the electron in the YDSE experiment. This is a result of the different space-time properties of the experiments as detailed in Table 3. For neutrino oscillations $\lambda^{e f f}$ is again different and depends on the propagators of both the source particle and the neutrinos.

The conclusion to be drawn from Table 3 is clear. In a strict, ontological, sense only the source and propagating particles exist as physical entities, and it is their particular 
properties together with the space-time structure of the production and detection events that determine $\phi_{B}-\phi_{A}$. The associated 'wave' is only a (sometimes useful, sometimes not) mathematical abstraction, as clearly stated long ago by E.J.Williams [64]

The electron is, of course, a particle. The wave is in the mathematics.

Although its inventor doubted it until the end of his days ${ }^{29}$ the photon is also a particle. The realisation of this is enough remove Einstein's mysterious and counter-intuitive postulate concerning the constancy of the velocity of light from the foundations of special relativity [66], and, by comparing photon properties with those of classical electromagnetic waves, to understand, in a new way, the basic concepts of quantum mechanics [67]. Also, all the mechanical aspects of classical electromagnetism may be quantitatively explained, at the quantum level, as an effect of the exchange of space-like virtual photons [68].

\section{Summary and Outlook}

In this paper Feynman's path amplitude formulation of quantum mechanics has been applied to several specific problems. In particular many of the optical experiments discussed in a qualitative way in Feynman's book 'QED' [1] have been calculated in detail. The problems of physical optics addressed include: the calculation, from first principles, of the refractive index of a transparent medium, the laws of refraction and reflection of light at the interface of two transparent media, the rectilinear propagation of light in a homogeneous transparent medium, Fermat's Principle, the reflection coefficient of light at normal incidence from the interface between two transparent media, and temporal dependence of the fringe visibility in a Michelson interferometer. The path amplitude method is also used to analyse quantum interference effects in Young double slit experiments (YDSE) using photons or electrons, in neutral-kaon quark flavour oscillations and in neutrino flavour oscillations. In all cases, except neutrino oscillations, agreement is found with the predictions of the classical wave theory of light or 'wave particle duality' (i.e. introducing the de Broglie wavelength of a massive particle in analogy with the wavelength of the photon), for the YDSEs, or, in the case of neutral kaon or neutrino flavour oscillations, solving the time-dependent Schrödinger Equation in the particle rest frame.

All of the calculations presented are straightforward applications of Feynman's five laws of quantum dynamics, that provide a description of causally-related elementary processes that succeed each other in space-time. These laws, presented and discussed in Section 2, are:

(I) The probabilistic interpretation of the Probability Amplitude (2.1).

(II) The law of Sequential Factorisation (2.2).

(III) The law of Quantum Mechanical Superposition (2.3).

\footnotetext{
${ }^{29}$ 'What are light quanta? Today every scoundrel believes he knows the answer, but he is wrong.' [65]
} 
(IV) The law of Composite Factorisation (2.4)

(V) The Feynman Path Integral (2.9)

The dynamics of the theory is entirely contained in V. The laws II-IV describe how the probabilty amplitude is constructed, while I gives its physical interpretation. The probability amplitude is built, according to II, as the product of a series of amplitudes describing elementary physical processes ordered in time. For the calculation of spacetime dependent interference effects, the most important of these process amplitudes are the Green functions, of space-time propagators, of particles in motion or at rest. The derivation of these propagators from, respectively, the Feynman Path Integral and the time-dependent Schrödinger Equation, is described in Section 3.

Before addressing specific problems of quantum interference, the physical interpretation of Feynman's formulation of quantum mechanics is discussed in some detail in Section 2. This interpretation is indeed quite different from that of previous formulations since the primary physical concept, the path amplitude, is neither a Hilbert-space state vector nor a wavefunction, and the concept of a particle, localised in space-time, as in classical physics, is also essential. Indeed consideration of classical propagation of particles localised in space-time is necessary for the correct calculation of the phases of the path amplitudes that are the physical basis of all space-time quantum interference phenomena. In this theory, particles, once created, propagate in space-time in a classical manner within each path amplitude. In the limit $\hbar \rightarrow 0$ the sum over paths is replaced by a single path, the one predicted by Hamilton's Principle of classical mechanics, and Feynman's laws I and II become a statement of Bayes theorem for the combination of conditional propabilities.

Shortcomings of the conventional text book 'wave packet' representation of particles and the associated misinterpretation of the space-time Heisenberg Uncertainty Relation are pointed out. The relation of Feynman's formulation to other 'interpretations' of quantum mechanics: Consistent Histories, The 'Many Worlds' interpretation and de Broglie's Pilot Wave theory are discussed. The much debated 'measurement problem' of the Heisenberg and Schrödinger formulations of quantum mecanics, seems, at least superficially, to be absent from Feynman's formulation. Perhaps new 'problems' remain to be discovered; but in any case, to the writer's best knowledge, no discussion of the 'philosophy' of Feyman's formulation yet exists.

In Section 4 it is demonstrated that Feynman's path amplitude formalism, in which the dynamics is entirely contained within the Green functions of particles, predicts, in a completely general manner, the classical wave theory of light, under a certain well-defined condition. The latter is that the lifetime of the photon source must be much longer than the photon path differences, in the experiment under consideration, divided by the velocity of light. This is the case for essentially all quantum optics experiments using a laser as light-source. All such experiments can then be correctly analysed using the classical wave theory of light, i.e. as done in well-known text books on optics [33, 37], in terms only of spatial intervals and photon wavelengths, without taking into account the times of the events corresponding to different observed physical processes. Although Feynman's formulation predicts the existence of an effective 'wave field' $U$ that satisifies Hemholtz' Equation (4.13), from which all predictions for interference and diffraction phenomena of 
the classical wave theory may be derived, the physical source of the phase of $U$ is solely the temporal propagator of the excited source atom, not the propagator of the photon itself. Thus any 'wave particle duality' in physical optics is between the photon ('particle') and its source ('wave') not between the 'particle' and 'wave' aspects of the photon itself. Indeed the latter does not exist in the path amplitude formulation. Thus, starting only from the quantum properties of particles (their Green functions) their 'wave' properties may be derived in full. It can seen that the latter are then, albeit useful, mathematical abstractions that do not exist in the same strictly ontological sense as the particles. Given the a priori existence of particles and Feynman's rules for their quantum behaviour, all their so-called 'wave' properties may be derived. The inverse procedure is evidently not possible. The fundamental physical entities are then the particles, not the 'waves'. In consequence there is in fact no 'wave particle duality' in the physical description, though by chance, in some cases such as in Davisson and Germers's original 'matter wave' experiment [69] or the electron Young double slit experiment, a naive application of this idea gives the same prediction as the full path amplitude calculation.

Finally in Section 4 it is demonstrated that the amplitude for forward diffractive scattering derived from the Helmholtz Equation by means of Green's theorem, can also be derived by direct summation of path amplitudes. The integration procedure used is similar to that employed in Section 5 to calculate the refractive index of a transparent medium in terms of the scattering amplitude of light by the atoms of the medium.

Section 5 contains three different calculations of the refractive index. In the first two it is assumed that the photon which interacts with the refractive medium is produced sufficently long after after the production time of the excited source atom that all geometrically allowed paths of the photon are possible. The first calculation assumes a sheet of refractive material so thin that, to a good approximation, the refractive index may be calculated by considering a single scattering of the photon from the atoms of the material. For this condition to be satisfied the sheet must be very thin - a small fraction of the photon wavelength. The second calculation removes this restriction and considers a block of uniform refractive material of arbitary thickness. In this case any number of scatterings of the photon from the atoms of the material are taken into account. The formula obtained for the refractive index is the same (5.9) as that obtained in the single scattering case. The third calculation considers photons produced and detected promptly after the production of the excited atom. In this case, causality forbids photon trajectories that deviate significantly from the classical straight line path between the source and the detector, reducing the number of possible interactions with the atoms of the refractive medium and therefore strongly reducing the refractive index - the so-called 'refraction annulment' effect. A simple experiment to detect this phenomenon (if indeed it exists) is shown in Fig.4.

In Section 6 the laws of refraction, reflection and rectilinear propagation of light are derived by imposing the condition that the phases of the probabilty amplitudes of suitably defined experiments are stationary for variations of the spatial location of the photon path from its source to the detector. The generalisation of the stationary phase condition to the motion of an arbitary physical object gives the quantum mechanical explanation of Hamilton's Principle of classical mechanics. Since the phase of the probability amplitude is proportional to the effective propagation time of a photon from its source to the detector 
Fermat's principle of least time is also derived. By considering the second derivatives of the phase of the probability amplitude, with respect to the positions and angles of the corresponding paths, the the size of the spread of photon trajectories around the classical path corresponding to a stationary phase, is estimated.

In Section 7 the experiment used in [1] to exemplify the application of quantum mechanics to optics, the reflection of light at normal incidence from the boundary of two transparent media with different refractive indices is analysed in detail for the case of a realistic experimental set-up. It is essentially the same as the first quantitative quantum mechanical experiment ever performed -Newton's study of his 'Rings' [2]. The result for the reflection coefficient at a vacuum/medium interface, (7.8) is significantly different from the formula (7.10), first derived by Fresnel, that is to be found in all text books on optics. For a refractive index $n=1.5$, the reflection coefficient predicted by the path amplitude calculation is 0.028 , some $40 \%$ smaller than the prediction, 0.040, of the Fresnel formula. Experimental discrimination between these two possiblities should be simple and straightforward. The present writer has been unable to find either in text books or in the published research literature any account of experimental verification of the Fresnel formula (7.10) for photons in the optical region of wavelength.

Fresnel originally derived his formula for the reflection coefficient from an elastic-solid model of light waves. The same formula is obtained in classical electromagnetism by applying surface boundary conditions to the electric and magnetic fields of the different electromagnetic waves in refractive media of different refractive indices. The problem with this approach may be that although a classical electromagnetic wave is, certainly, a high density beam of monochromatic photons, a single photon is, equally certainly, not a classical electromagnetic wave, so it makes no sense to associate with it classical electric and magnetic fields. If the Feynman space-time picture of reflection is correct, the essential physics of the process relates not to the interface between media but resides in the amplitude $\mathcal{A}_{\text {scat }}$ for elastic scattering of photons by the atoms in the interior of the medium, as is manifest in the derivation of the formula (5.7) for the refractive index. This formula, and the formula (7.8) for the reflection coefficient are derived in exactly the same way by by integrating the path amplitudes corresponding to the scattering of the photon over all of the atoms of the refractive material probed by the photon in the experiment. The interface occurs only geometrically as a limit on this spatial integration.

This being said, it is certainly possible to envisage an experiment where the Fresnel formula would be appropriate and correct. For example, the reflection of a beam of microwave photons of sufficiently high spatial density from the surface of a dielectric. In this case the description of the beam as an electromagnetic wave will be appropriate, and the molecules of different dielectrics will be differently polarised, over macroscopic distances, by the electric fields associated with the microwave beam. The waves in different media will have different characteristics related by the boundary conditions on the electric fields of the waves, so that the Fresnel formula for the reflection coefficient should be correct, and the scattering amplitude of individual microwave photons from individual molecules less directly relevant. On the other hand, the macroscopic dielectric polarisation effects for a single optical photon entering a block of glass are clearly negligible and a purely quantum mechanical description is mandatory. For a general discussion of the different circumstances in which either the classical electromagnetic wave (high photon 
density) or the quantum mechanical (low photon density) description is appropriate, see $[67]$

The experiment described in Section 8, measurement of the temporal dependence of the fringe visiblity in a Michelson interferometer is one in which path length differences are deliberately chosen to be of the order of, or greater than, $c \tau_{S}$ where $\tau_{S}$ is the mean lifetime of the excited atomic state that produces the observed photon. The classical wave theory of light, in which only spatial intervals are considered is therefore unable, in principle, to describe such an experiment. Thus new predictions specific to the path amplitude method are obtained. These predictions for the fringe visiblity as a function ot the time elapsed after the production of the excited source atom are shown, for different path length differences, in Fig.9. The path amplitude calculation of the effects of random source motion on the time-integrated fringe visibility found in Appendix E predicts that there should be no effect on the fringe visiblity from this source. This is in contradiction with the prediction, (8.14), of Rayleigh, based on the classical wave theory, that such 'Doppler broadening' effects should be large ${ }^{30}$. In order to test the path amplitude prediction of a vanishing effect due to source motion, experiments are required that clearly discriminate this effect from that of pressure broadening. So far this has not been done.

In Section 9 analagous 'two probability amplitude' experiments in photon or electron optics, neutral kaon oscillations and neutrino oscillations are analysed and compared. The understanding of the different results obtained, summarised in Table 3, requires careful consideration of the nature of the particle source and the particles themselves as well as the space-time structure of the production and detection events of the different path amplitudes. Only for the photon and electron YDSE does the simple "wave particle duality' concept, that is the introduction of the de Broglie wavelength of the photon or the electron, give the same result as the path amplitude analysis. For the neutral kaon and neutrino oscillation cases, quite different effective wavelengths are found for the associated 'matter waves'. For optical interference experiments the source of the 'wave' is solely the propagator of the excited source atom. As neutrino oscillation experiments (unlike the electron YDSE and neutral kaon oscillation experiments) have a similar excited source particle, it is clear, if the path amplitude description is indeed correct, that the source particle must give an important contribution to the oscillation phase also in this case. In addition there is the contribution of the propagators of the neutrinos themselves, which is analogous to the sole source of the interference phase in the electron YDSE and neutral kaon oscillation experiments. Before the work reported in $[11,12,13]$ only this last contribution to the neutrino oscillation phase was taken into account.

Although all optical and massive particle diffraction and interference experiments that have been performed to date and found to be agreement with the predictions of the classical wave theory of light, or 'wave particle duality' can be interpreted, following the arguments given in Section 4 and 9, as evidence for the correctness of Feynman's formulation of quantum mechanics, the present paper contains several new predictions. If any of these predictions is not confirmed by experiment, Feynman's space-time formulation of quantum mechanics is not of general validity and answers must be sought to the question

\footnotetext{
${ }^{30}$ Indeed, as shown by the entries in the first and last rows of Table 1, the damping predicted by the Rayleigh formula is too large to explain Michelson's fringe visiblity measurements for the $\mathrm{H}_{r} 3 \mathrm{p}-2 \mathrm{~s}$ and $\mathrm{Na} D$ 3p-3s transitions.
} 
why, in some cases, cited above, the predictions agree with experiment, and in others not. These new predictions are:

(i) The refraction annulment effect (Section 5).

(ii) The reflection coefficient of light at normal incidence (Section 7).

(iii) Time dependence of fringe visiblity in the Michelson interferometer (Section 8)

(iv) Absence of source motion damping of fringe visiblity in the Michelson interferometer (Section 8).

(v) Different oscillation lengths for ' $\nu_{\mu}$ disappearence' using $\pi \rightarrow \mu \nu$ or $\mathrm{K} \rightarrow \mu \nu$ as neutrino sources.

It is clearly of interest to apply the methods developed in the present paper to other related physical problems. Some examples are:

(A) The coefficients of reflection and refraction of light at non-normal incidence. For this it is necessary to take into account photon polarisation. This requires, for the path amplitude method, a complete quantum description of polarisation effects in both the photon production process (electric dipole radiation from a polarised source atom) as well as photon scattering processes (Rayleigh scattering of polarised photons). Another interesting related problem is the path amplitude description of bi-refringence.

(B) Heavy quark flavour oscillations for entangled systems. Examples are:

$$
\phi \rightarrow \mathrm{K}_{\mathrm{L}} \mathrm{K}_{\mathrm{S}} \quad \Upsilon(4 S) \rightarrow \mathrm{B}_{\mathrm{H}} \mathrm{B}_{\mathrm{L}}
$$

The latter is of considerable current interest due to the on-going experimental programs of the BABAR and BELLE b-factories. Unlike the 'unentangled' process $\pi^{-} p \rightarrow \Lambda\left(\mathrm{K}_{\mathrm{L}}, \mathrm{K}_{\mathrm{S}}\right)$ described above, both the $\phi$ and the $\Upsilon(4 S)$ are coherent sources, similar to an excited atom in physical optics. The corresponding values of $\Delta t_{S L}$, $\Delta t_{L H}$ calculated using $(9.41)$ are $6.12 \times 10^{-25} \mathrm{~s}, 9.55 \times 10^{-26}$ s repectively, where the value $m_{H}-m_{L}=3.28 \times 10^{-10} \mathrm{Mev} / \mathrm{c}^{2}$ [56] has been used for the neutral b-meson mass difference. These time differences may be compared with the mean lifetimes of the $\phi$ and the $\Upsilon(4 S)$ source particles of $1.5 \times 10^{-22} \mathrm{~s}$ and $4.7 \times 10^{-23} \mathrm{~s}$ [56], which are factors of 24.5 and 492 times greater than $\Delta t_{S L}$ and $\Delta t_{L H}$. Thus, unlike for the case of $\pi^{-} p \rightarrow \Lambda\left(\mathrm{K}_{L}, \mathrm{~K}_{S}\right)$ neutral $\mathrm{K}$ - or b-mesons can, in principle, have the same detection time in alternative histories with different mass eigenstates, provided that, as in physical optics or neutrino oscillations, the particles are produced at different times in the alternative histories. In view of more stringent space-time restrictions imposed by the requirement of observation of both final state particles it is possible that the off-shell nature of the propagating particles is more important, in this case, in enabling interference, rather than production time differences or velocity smearing due to radiative effects. Only an actual calculation can show if this is the case or not. 
(C) Effective refractive indices for neutrinos. It is important, in view of the great current interest in neutrino oscillation experiments to repeat the refractive index calculations of Section 5 above for the case of neutrinos interacting with matter. The model used till now to derive the effective refractive indices of neutrinos in bulk matter $[70,71]$ was constructed by analogy with that used to used to describe coherent regeneration [72] of neutral kaons for which (see Table 3 above) simple 'wave particle duality' is a good approximation to path amplitude calculations. However, in the neutrino case, the path amplitude calculation suggests that this analogy breaks down, and an important contribution to the interference phase originates in the propagator of the source particle. This effect has not, to date, been included in the calculation of neutrino refractive indices.

One of the precepts of the standard 'Copenhagen Interpretation' of quantum mechanics is that our direct exerience of the world can only be understood, in a rational manner, by an appeal to classical concepts

The quantum theory is characterised by the acknowledgement of a fundamental limitation in the classical physical ideas when applied to atomic phenomena. The situation then created is of a peculiar nature, since our interpretation of the experimental material rests essentially upon the classical concepts [9].

Is it indeed true that '...our interpretation of the experimental material rests essentially upon the classical concepts'? In the case of one 'experiment' that is performed by every human being ${ }^{31}$ every day of her (or his) life without she (or he) being consciously aware of it, this statement seems to be quite untrue. The 'experiment' in question is our perception of colour. The photon detector D, forming an essential part of all the optical experiments discussed in Sections 2-8 above, was never precisely specified, but could well be one of the three types of cone receptor cells in the retina of the human eye. The detection efficiency of these receptors for photons is similar to that of a photo-diode with a flat spectral response in the optical region preceded by wide-band wavelength filters with roughly Gaussian acceptance profiles centered at $\lambda_{\gamma} \simeq 440,540$ and $570 \mathrm{~nm}$ with full widths at half-maximum $\simeq 200,200$ and 300nm [73]. What we perceive as the colour 'green' corresponds not always to some discrete or narrow range of values of $\lambda_{\gamma}$ but in general to a weighted average (by the human nervous system) of the intensities of photons in different spectral regions that are incident on the three types of receptors. The 'interpretation of the experimental material' mentioned above by Bohr when this material is, say, the perception of the colour green or the colour red, varies enormously according to the circumstances, but in all cases this interpretation is possible only in terms of quantum, not classical, concepts. The sources of the photons for all the colour perception experiments that will now be discussed are excited atoms in the Sun. Myriads of different spectral lines produced by spontaneous decay of these atoms are responsible for the 'white light' arriving at the surface of the Earth in daytime. The experiments differ in exactly how certain of these photons come to impinge on the cone receptors of the retina.

I step out on to my balcony and look at a geranium. I see that its leaves are green and

\footnotetext{
${ }^{31}$ Colour-blind people excepted
} 
its flowers red. Why? The explanation is the different atomic structures of the matter of the leaves and flowers. Photons of some wavelengths are absorbed, others re-emitted as fluorescent radiation by these atoms. The re-emitted radiation, incident on the retinal receptors and suitably re-weighted gives the perception of green for the leaves and red for the flowers. In this case the physics explanation of colour vision is simple, the different parts of the geranium filter, in a different way, the sunlight photons that are incident on it.

Nearby there is a shower of rain, and the Sun is lowering towards the western horizon. I look at the eastern sky and, at certain angles, see the green and red components of a rainbow. In this case the physics explanation of my perception of these colours is quite different. Now the photons responsible for the sensations of 'green' and 'red' have in each case closely similar wavelengths. The reason why they are observed in different spatial directions is explained by the phenomena analysed in Sections 5 and 6 above -refraction and reflection by spherical rain-drops. The angles at which the green and red bows are observed are controlled by the values of the scattering amplitude $\mathcal{A}_{\text {scat }}$ and the wavelength $\lambda_{\gamma}^{0}$ in (5.9) causing different wavelength photons to follow diffent paths according to Snell's law of refraction, (6.11).

Now I take a compact disc (CD) and hold it in the direct sunlight, turning it in my hand. At certain angles of the face of the CD relative to the direction of the Sun I observe vivid flashes of green or red light. As for the rainbow, the photons striking the cone receptors in my retina, when I perceive the green or red colour, occur in narrow bands of wavelength. However, the physical source of this sensation of colour is yet again different. The face of the CD constitutes a diffraction grating. The bright flashes of colour correspond to constructive interference of the path amplitudes of photons for particular intervals of wavelength. The quantum mechanical description is essentially the same as for the photon YDSE described in Section 9, except that, not just two, but many, probability amplitudes interfere constructively [74] to give large values of the combined probability amplitude $A_{f i}$ in (2.1) and therefore a large signal in the output neurones of the cone cell receptors. Where is now the physical origin of the visual perception of the different colours? It is not simply the grooves on the surface of the CD. These serve simply to define the lengths of the photon paths from their begining, at an atom in the Sun, to their end, at a cone cell in my retina. Constructive interference corresponds to a constant phase difference between the amplitudes of photon paths reflected between successive grooves on the CD. In Feynman's interpretation of quantum mechanics, these phase diffences are those between alternative paths of the same photon reflected from different places on the surface of the CD in its alternative histories. Constructive interference occurs when, for each adjacent pair A,B of paths, $\phi_{B}-\phi_{A}=2 \pi n$ where $n$ is an integer. The actual physical source of this phase difference is, uniquely, the temporal propagator $(2.12),(3.20)$ of the source atom. Thus, as I move the CD to observe either green or red interference fringes I select photons from different sets of source atoms in the Sun, and am, in fact, observing the temporal phase advances of the wavefunctions of these different atoms (Feynman's 'stopwatch hand' [1]) as probed by alternative paths followed by each single photon that is absorbed in the cone cells of my retina. These phase advances actually occur some 8.3 minutes before I perceive the fringes!

The last experiment on the perception of colour to be described is the one featured 
on the dust-jacket of the first edition of Feynman's book [1], that provided the primary motivation for the writing of the present paper. In order to observe this effect -thin film interference, under the best conditions, an overcast, rainy, day is desirable. This is for two reasons, firstly because wet tarmac road surfaces favour the production of thin films when small oil-drops fall upon them from passing vehicles, and secondly because diffuse sunlight, multiply scattered and refracted from the water vapour droplets of clouds, ensures that photons with paths that satisfy conditions for constructive or destructive interference can arrive at the eye from all parts of the thin oil-patch. In this case, the perceived colour is affected both by refraction, as in the case of the rainbow, and by path differences as in the case of the photon YDSE, or the CD experiment. The angular positions at which the differently coloured 'Newton's Rings' appear depend on both the thickness of the oil film and, as Newton noted [2], on the refractive index of the oil. However, the basic physical mechanism, in Feynman's interpretation, is the same as for the CD experiment temporal phase advances of the wavefunctions of atoms in the Sun, several minutes before the photons from the decay of those atoms strike the receptor cells of the retina of the observer.

Indeed, quantum mechanics is essential not only for 'the interpretation of the experimental material' but to the very physical mechanism of colour vision itself in the eye and the brain. The cone cell receptors are quite analagous to the leaves and flowers of the geranium i.e. they function essentially as wavelength filters. They do this because of the different structures of atomic energy levels to be found in the three different types of cone receptor cells. The world of our every-day experience not only requires quantum mechanics for its rational interpretation but also for enregistering the raw data from which that experience is derived. 


\section{Appendix A}

The case of a rectangular boundary, of sides $L_{Y}, L_{Z}$ for the refractive medium in Fig. 2 or Fig. 3 is shown in Fig 12a. The Ox axis, along which are situated the excited source atom and the photon detector, intersects the $Y Z$ plane at $\mathrm{O}_{P}$ and has cartesian coordinates $(Z, Y)$ relative to the center, $\mathrm{O}_{R}$, of the rectangular boundary. The axes OY and $\mathrm{OZ}$ are parallel to the sides of the rectangle $B_{1} B_{2} B_{3} B_{4}$ whose plane is perpendicular to Ox. The typical boundary point, $B$, has polar coordinates $\left(R_{1}, \phi_{1}\right)$ relative to $\mathrm{O}_{P}$ The corresponding value of $r_{1}^{\max }$ in (5.2) (see also Fig.2) is given by:

$$
r_{1}^{\max }=\sqrt{x_{1}^{2}+R_{1}^{2}}=x_{1}+\frac{R_{1}^{2}}{2 x_{1}}+O\left(\frac{R_{1}^{4}}{x_{1}^{3}}\right)
$$

The angles $\Phi_{1}, \Phi_{2}, \Phi_{3}$ and $\Phi_{4}$, of the line segments $O_{P} B_{1}, O_{P} B_{2}, O_{P} B_{3}$ and $O_{P} B_{4}$, joining the corners of the rectangle to $\mathrm{O}_{P}$, are defined, in an anti-clockwise sense, relative to the axis OZ,.

The function $r_{1}^{\max }\left(d_{i}, \phi_{1}\right)$ in (5.2) then has the following form, where corrections of $O\left(R_{1}^{4} / x_{1}^{3}\right)$ are neglected:

$$
\begin{gathered}
\Phi_{4}<\phi_{1}<2 \pi+\Phi_{1} \\
r_{1}^{\max }\left(x_{1}, L_{Z}, Z, \phi_{1}\right)=x_{1}+\frac{\left(L_{Z} / 2-Z\right)^{2}}{2 x_{1} \cos ^{2} \phi_{1}} \\
\Phi_{1}<\phi_{1}<\Phi_{2} \\
r_{1}^{\max }\left(x_{1}, L_{Y}, Y, \phi_{1}\right)=x_{1}+\frac{\left(L_{Y} / 2-Y\right)^{2}}{2 x_{1} \sin ^{2} \phi_{1}} \\
\Phi_{2}<\phi_{1}<\Phi_{3} \\
r_{1}^{\max }\left(x_{1}, L_{Z}, Z, \phi_{1}\right)=x_{1}+\frac{\left(L_{Z} / 2+Z\right)^{2}}{2 x_{1} \cos ^{2} \phi_{1}} \\
\Phi_{3}<\phi_{1}<\Phi_{4} \\
r_{1}^{\max }\left(x_{1}, L_{Y}, Y, \phi_{1}\right)=x_{1}+\frac{\left(L_{Y} / 2+Y\right)^{2}}{2 x_{1} \sin ^{2} \phi_{1}}
\end{gathered}
$$

The case of a circular boundary is shown in Fig 12b. By symmetry, it is sufficient to consider the displacement, $Y$, of of the projection, $\mathrm{O}_{P}$, of the $\mathrm{Ox}$ axis from the center of the circle, $\mathrm{O}_{C}$, whose plane is perpendicular to Ox. Choosing the OY axis parallel 


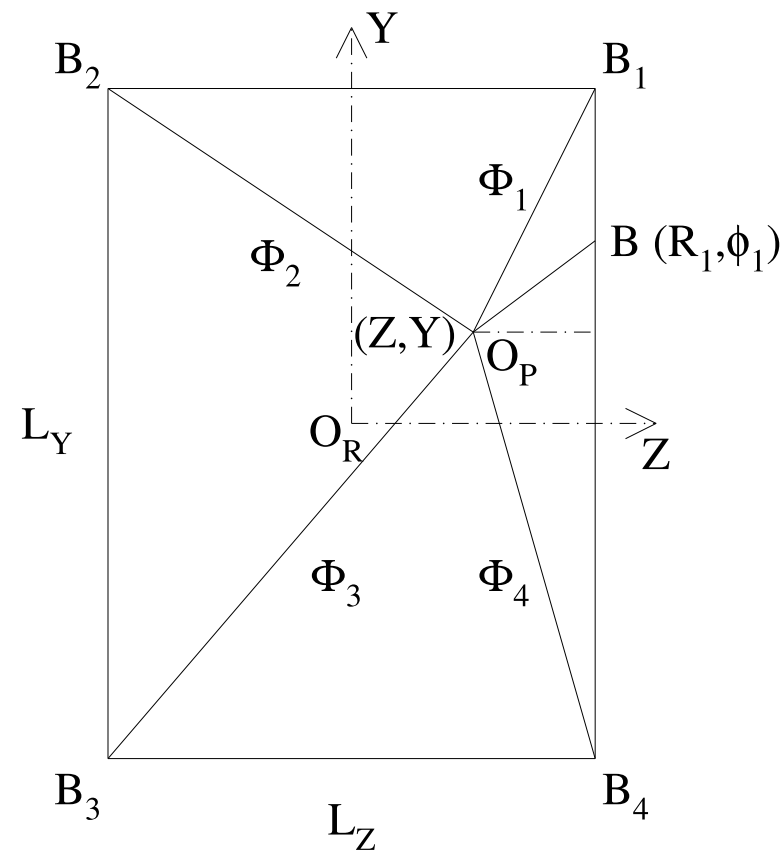

a)

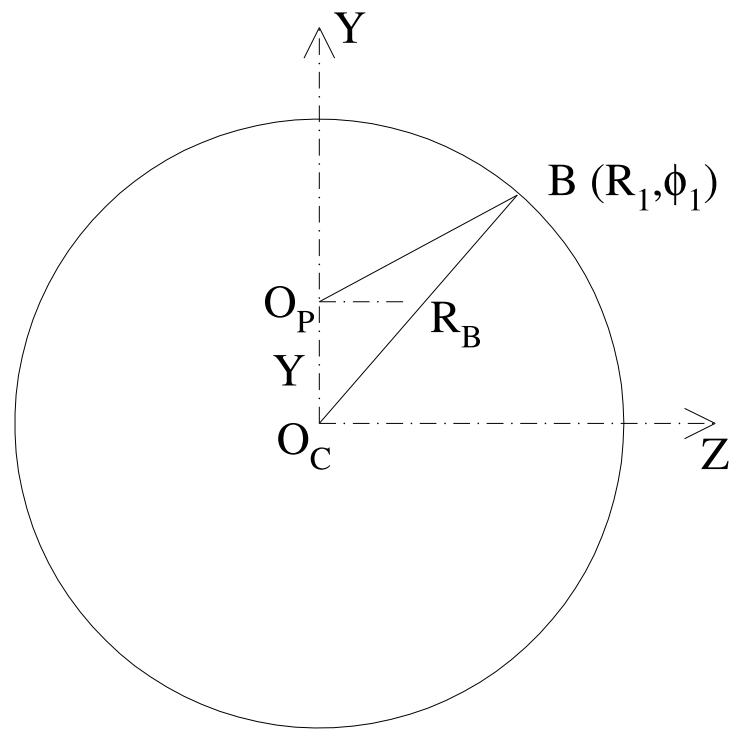

b)

Figure 12: Dependence of the upper limits of the $r_{1}$ integral in (5.2) on the azimuthal angle $\phi_{1}$ for different boundary geometries. $O_{P}$ is the projection of the classical rectilinear path joining the source and detector on the $Y Z$ (transverse) plane. Various geometrical parameters used to specify $r_{1}^{\max }$ are defined for the case of rectangular, a), and circular, b), boundaries. 
to $O_{C} O_{P}$, and a boundary point, $B$, with polar coordinates $\left(R_{1}, \phi_{1}\right)$ relative to $\mathrm{O}_{P}$, the function $r_{1}^{\max }\left(d_{i}, \phi_{1}\right)$ is found, from the geomery of Fig.10b, to be:

$$
r_{1}^{\max }\left(x_{1}, R_{B}, Y, \phi_{1}\right)=x_{1}+\frac{\left(\sqrt{R_{B}^{2}-Y^{2} \cos ^{2} \phi_{1}}-Y \sin \phi_{1}\right)^{2}}{2 x_{1}}
$$

where $R_{B}$ is the radius of the circular boundary.

The following contributions of the upper limit of the $r_{1}$ integral in (5.2) are then found :

$$
\begin{gathered}
I_{U}=-\int_{0}^{2 \pi} \exp \left[i \kappa r_{1}^{\max }\left(d_{i}, \phi_{1}\right) d \phi_{1}\right. \\
=-\int_{0}^{2 \pi}\left[\cos \frac{2 \pi r_{1}^{\max }\left(d_{i}, \phi_{1}\right)}{\lambda_{\gamma}^{0}}+i \sin \frac{2 \pi r_{1}^{\max }\left(d_{i}, \phi_{1}\right)}{\lambda_{\gamma}^{0}}\right] d \phi_{1}
\end{gathered}
$$

where

$$
\begin{array}{cr}
d_{i}=x_{1}, L_{Y}, L_{Z}, Y, Z & \text { rectangular boundary } \\
d_{i}=x_{1}, R_{B}, Y \quad \text { circular boundary }
\end{array}
$$

In the typical case that $r_{1}^{\max } \gg \lambda_{\gamma}^{0}$, a very rapid phase variation of the integrand in (A7) occurs as a function of $\phi_{1}$, so that the integral $I_{U}$ over this variable vanishes.

An possible exception to this is the case $Y=0$ in (A6), in which case:

$$
r_{1}^{\max }\left(x_{1}, R_{B}, 0, \phi_{1}\right)=x_{1}+\frac{R_{B}^{2}}{2 x_{1}}
$$

which is independent of $\phi_{1}$, so that no cancellations due to rapid phase variation as a function of this variable occur. However, even in this case, both the position of $\mathrm{O}_{P}$ must be specified, and the boundary radius $R_{B}$ must be constant, to within a fraction of $\lambda_{\gamma}^{0}$ in order to avoid the cancellation of $I_{U}$ due to rapid phase variation of the sine and cosine functions in (A7). 


\section{Appendix B}

The nested $n$ fold integral $\mathcal{I}_{n}(L)$ is defined as:

$$
\mathcal{I}_{n}(L)=\int_{x_{2}}^{\bar{x}+\frac{L}{2}} d x_{1} \int_{x_{3}}^{\bar{x}+\frac{L}{2}} d x_{2} \ldots \int_{\bar{x}-\frac{L}{2}}^{\bar{x}+\frac{L}{2}} d x_{n}
$$

Consider the incremental change: $L \rightarrow L+\delta L$. Substitution in (B1) gives:

$$
\begin{aligned}
\mathcal{I}_{n}(L+\delta L)= & \left(\int_{x_{2}}^{\bar{x}+\frac{L}{2}} d x_{1}+\int_{\bar{x}+\frac{L}{2}}^{\bar{x}+\frac{L+\delta L}{2}} d x_{1}\right)\left(\int_{x_{3}}^{\bar{x}+\frac{L}{2}} d x_{2}+\int_{\bar{x}+\frac{L}{2}}^{\bar{x}+\frac{L+\delta L}{2}} d x_{2}\right) \\
& \ldots\left(\int_{\bar{x}-\frac{L+\delta L}{2}}^{\bar{x}-\frac{L}{2}} d x_{n}+\int_{\bar{x}-\frac{L}{2}}^{\bar{x}+\frac{L}{2}} d x_{n}+\int_{\bar{x}+\frac{L}{2}}^{\bar{x}+\frac{L+\delta L}{2}} d x_{n}\right) \\
= & \mathcal{I}_{n}(L)+i_{1}+i_{2}+\ldots+i_{j}+\ldots+i_{n}+i_{-}+O\left(\delta L^{2}\right) \quad(B 2)
\end{aligned}
$$

where

$$
\begin{aligned}
i_{1} & =\frac{\delta L}{2} \int_{x_{3}}^{\bar{x}+\frac{L}{2}} d x_{2} \int_{x_{4}}^{\bar{x}+\frac{L}{2}} d x_{3} \ldots \int_{\bar{x}-\frac{L}{2}}^{\bar{x}+\frac{L}{2}} d x_{n}=\frac{\delta L}{2} \mathcal{I}_{n-1}(L) \\
i_{j} & =\int_{x_{2}}^{\bar{x}+\frac{L}{2}} d x_{1} \int_{x_{3}}^{\bar{x}+\frac{L}{2}} d x_{2} \ldots \int_{\bar{x}+\frac{L}{2}}^{\bar{x}+\frac{L+\delta L}{2}} d x_{j} \ldots \int_{\bar{x}-\frac{L}{2}}^{\bar{x}+\frac{L}{2}} d x_{n} \\
i_{-} & =\int_{x_{2}}^{\bar{x}+\frac{L}{2}} d x_{1} \int_{x_{3}}^{\bar{x}+\frac{L}{2}} d x_{2} \ldots \int_{x_{n}}^{\bar{x}+\frac{L}{2}} d x_{n-1} \int_{\bar{x}-\frac{L+\delta L}{2}}^{\bar{x}-\frac{L}{2}} d x_{n}
\end{aligned}
$$

Because of the linear dependence of each integral on its upper and lower limits, the following replacement may be made in (B4):

$$
\ldots \int_{x_{j}}^{\bar{x}+\frac{L}{2}} d x_{j-1} \int_{\bar{x}+\frac{L}{2}}^{\bar{x}+\frac{L+\delta L}{2}} d x_{j} \ldots \rightarrow \ldots \int_{\bar{x}_{j}}^{\bar{x}+\frac{L}{2}} \frac{\delta L}{2} \ldots
$$

where

similarly

$$
\bar{x}_{j}=\bar{x}+\frac{L}{2}+\frac{\delta L}{4}
$$

$$
\ldots \int_{\bar{x}_{j-1}}^{\bar{x}+\frac{L}{2}} d x_{j-2} \int_{\bar{x}_{j}}^{\bar{x}+\frac{L}{2}} \frac{\delta L}{2} \ldots \rightarrow \ldots \int_{\bar{x}_{j-1}}^{\bar{x}+\frac{L}{2}} \frac{\delta L}{4} \frac{\delta L}{2} \ldots
$$

where

$$
\bar{x}_{j-1}=\bar{x}+\frac{L}{2}+\frac{\delta L}{8}
$$

It follows that $i_{2}, i_{3}, \ldots i_{n}$ are at least of order $\delta L^{2}$. Similar considerations show that:

$$
i_{-}=\int_{x_{2}}^{\bar{x}+\frac{L}{2}} d x_{1} \int_{x_{3}}^{\bar{x}+\frac{L}{2}} d x_{2} \ldots \int_{\bar{x}_{n}}^{\bar{x}+\frac{L}{2}} d x_{n-1} \frac{\delta L}{2}
$$

where

or

$$
\bar{x}_{n}=\bar{x}-\frac{L}{2}-\frac{\delta L}{4}
$$

$$
\begin{aligned}
i_{-} & =\int_{x_{2}}^{\bar{x}+\frac{L}{2}} d x_{1} \int_{x_{3}}^{\bar{x}+\frac{L}{2}} d x_{2} \ldots\left(\int_{\bar{x}-\frac{L}{2}}^{\bar{x}+\frac{L}{2}} d x_{n-1}+\int_{\bar{x}-\frac{L}{2}-\frac{\delta L}{4}}^{\bar{x}-\frac{L}{2}} d x_{n-1}\right) \frac{\delta L}{2} \\
& =\frac{\delta L}{2} \mathcal{I}_{n-1}(L)+O\left(\delta L^{2}\right) \quad(B 7)
\end{aligned}
$$


Since $i_{2}, i_{3}, \ldots i_{n}$ give no $O(\delta L)$ contribution, combining (B2),(B3) and (B7) then gives:

$$
\mathcal{I}_{n}(L+\delta L)=\mathcal{I}_{n}(L)+\mathcal{I}_{n-1} \delta L+O\left(\delta L^{2}\right)
$$

or, from the Taylor expansion of $\mathcal{I}_{n}(L+\delta L)$,

$$
\frac{d \mathcal{I}_{n}(L)}{d L}=\mathcal{I}_{n-1}(L)
$$

hence

$$
\mathcal{I}_{n}(L)=\int_{0}^{L} \mathcal{I}_{n-1}(L) d L \quad(B 10)
$$

Since

$$
\mathcal{I}_{1}(L)=\int_{\bar{x}-\frac{L}{2}}^{\bar{x}+\frac{L}{2}} d x_{1}=L
$$

then

$$
\begin{aligned}
\mathcal{I}_{2}(L)= & \int_{0}^{L} L d L=\frac{L^{2}}{2} \\
\mathcal{I}_{3}(L)= & \int_{0}^{L} \frac{L^{2}}{2} d L=\frac{L^{3}}{3 !} \\
\mathcal{I}_{4}(L)= & \int_{0}^{L} \frac{L^{3}}{3 !} d L=\frac{L^{4}}{4 !} \\
& \ldots \\
\mathcal{I}_{n}(L)= & \int_{0}^{L} \frac{L^{n-1}}{(n-1) !} d L=\frac{L^{n}}{n !} \quad(B 12)
\end{aligned}
$$

which is (5.16) of the text. 


\section{Appendix C}

The nested $r_{1}, r_{2}, r_{3}$ integral in (4.30) is:

$$
\mathcal{I}_{3}(r)=\int_{x_{1}-x_{2}}^{\Delta s-r_{2}-r_{3}+x_{1}} e^{i \kappa r_{1}} d r_{1} \int_{x_{2}-x_{3}}^{\Delta s-r_{3}+x_{2}} e^{i \kappa r_{2}} d r_{2} \int_{x_{3}}^{\Delta s+x_{3}} e^{i \kappa r_{3}} d r_{3}
$$

Performing the $r_{1}$ integral:

$$
\mathcal{I}_{3}(r)=\frac{e^{i \kappa x_{1}}}{i \kappa} e^{i \kappa r_{1}} d r_{1} \int_{x_{2}-x_{3}}^{\Delta s-r_{3}+x_{2}}\left[e^{i \kappa\left(\Delta s-r_{3}\right)}-e^{i \kappa\left(r_{2}-x_{2}\right)}\right] d r_{2} \int_{x_{3}}^{\Delta s+x_{3}} e^{i \kappa r_{3}} d r_{3}
$$

Performing the $r_{2}$ integral:

$$
\mathcal{I}_{3}(r)=e^{i \kappa x_{1}} \int_{x_{3}}^{\Delta s+x_{3}}\left[\frac{\left(\Delta s-r_{3}+x_{3}\right)}{i \kappa} e^{i \kappa \Delta s}-\frac{\left(e^{i \kappa \Delta s}-e^{i \kappa\left(r_{3}-x_{3}\right)}\right)}{(i \kappa)^{2}}\right] d r_{3}
$$

Finally, performing the $r_{3}$ integral:

$$
\mathcal{I}_{3}(r)=e^{i \kappa x_{1}}\left(\frac{i}{\kappa}\right)^{3}\left[\left(-\frac{(i \kappa \Delta s)^{2}}{2}+i \kappa \Delta s-1\right) e^{i \kappa \Delta s}+1\right]
$$

The factor $\exp \left[i \kappa x_{1}\right]$ is cancelled by the factor $\exp \left[-i \kappa x_{1}\right]$ in (5.30) so that the integrand of the $x_{1}, x_{2}$ and $x_{3}$ integrals in this equation is constant. The integral is then given by (5.16) of the text as the $r$ and $x$ integrals factorise. The replacement $\kappa \Delta s=\Delta \Phi$ in (C1) yields (5.31) of the text.

The general expression for the nested $n$-fold $r$ and $x$ integrals obtained in a similar manner to the three-fold case just discussed is, in the notation of (5.33):

$\mathcal{I}_{n}(x, r)=\frac{(i \beta L)^{n}}{n !}\left\{\left((-1)^{n} \frac{(i \Delta \Phi)^{n-1}}{(n-1) !}-(-1)^{n} \frac{(i \Delta \Phi)^{n-2}}{(n-2) !}+\ldots-\frac{(i \Delta \Phi)^{2}}{2}+i \Delta \Phi-1\right) e^{i \Delta \Phi}+1\right\}$ 


\section{Appendix D}

Multiplying $\exp [i \Delta \Phi]$ by the polynomials in $i \Delta \Phi$ at each order in $\beta L$, the expresion in large curly brackets of (5.33) may be written as:

$$
\begin{aligned}
F_{\text {ref }} & =1-i \beta L\left[i \Delta \Phi+\frac{(i \Delta \Phi)^{2}}{2 !}+\frac{(i \Delta \Phi)^{3}}{3 !}+\ldots\right] \\
& +\frac{(i \beta L)^{2}}{2 !}\left[(i \Delta \Phi)^{2}\left(\frac{1}{1 !}-\frac{1}{2 !}\right)+(i \Delta \Phi)^{3}\left(\frac{1}{2 !}-\frac{1}{3 !}\right)+\ldots\right] \\
& +\frac{(i \beta L)^{3}}{3 !}\left[(i \Delta \Phi)^{3}\left(-\frac{1}{2 !}+\frac{1}{2 !}-\frac{1}{3 !}\right)+(i \Delta \Phi)^{4}\left(-\frac{1}{2 ! 2 !}+\frac{1}{3 !}-\frac{1}{4 !}\right)+\ldots\right] \\
& +\frac{(i \beta L)^{4}}{4 !}\left[(i \Delta \Phi)^{4}\left(\frac{1}{3 !}-\frac{1}{2 ! 2 !}+\frac{1}{3 !}-\frac{1}{4 !}\right)+(i \Delta \Phi)^{5}\left(\frac{1}{3 ! 2 !}-\frac{1}{2 ! 3 !}+\frac{1}{4 !}-\frac{1}{5 !}\right)+\ldots\right]+\ldots \\
& +\frac{(i \beta L)^{n}}{n !}\left[(i \Delta \Phi)^{n}\left(\ldots-\frac{1}{(n-2) ! 2 !}+\frac{1}{(n-1) !}-\frac{1}{n !}\right)\right. \\
& \left.+(i \Delta \Phi)^{n+1}\left(\ldots-\frac{1}{(n-1) ! 2 !}+\frac{1}{n !}-\frac{1}{(n+1) !}\right)+\ldots\right] \\
& +\ldots
\end{aligned}
$$

Separating out the real and imaginary parts at each order in $\beta L$ the gives the expression:

$$
\begin{aligned}
& F_{r e f}=1+\beta L\left[\Delta \Phi-\frac{(\Delta \Phi)^{3}}{3 !}+\ldots+i\left(\frac{(\Delta \Phi)^{2}}{2 !}-\frac{(\Delta \Phi)^{4}}{4 !}+\ldots\right)\right] \\
&+\frac{(\beta L)^{2}}{2 !}\left[\Delta \Phi\left(\Delta \Phi-\frac{(\Delta \Phi)^{3}}{3 !}+\ldots\right)-\frac{(\Delta \Phi)^{2}}{2 !}+\frac{(\Delta \Phi)^{4}}{4 !}-\ldots\right. \\
&+i\left(\frac{(\Delta \Phi)^{3}}{3 !}-\frac{(\Delta \Phi)^{5}}{5 !}+\ldots+\Delta \Phi\left(\frac{(\Delta \Phi)^{2}}{2 !}-\frac{(\Delta \Phi)^{4}}{4 !}+\ldots\right)\right] \\
&+\frac{(\beta L)^{3}}{3 !}\left[\frac{(\Delta \Phi)^{3}}{3 !}-\frac{(\Delta \Phi)^{5}}{5 !}+\ldots+\Delta \Phi\left(-\frac{(\Delta \Phi)^{2}}{2 !}+\frac{(\Delta \Phi)^{4}}{4 !}-\ldots\right)+\frac{(\Delta \Phi)^{2}}{2 !}\left(\Delta \Phi-\frac{(\Delta \Phi)^{3}}{3 !}+\ldots\right)\right. \\
&\left.+i\left(\frac{(\Delta \Phi)^{4}}{4 !}-\frac{(\Delta \Phi)^{6}}{6 !}+\ldots+\Delta \Phi\left(-\frac{(\Delta \Phi)^{3}}{3 !}+\frac{(\Delta \Phi)^{5}}{5 !}-\ldots\right)+\frac{(\Delta \Phi)^{2}}{2 !}\left(\frac{(\Delta \Phi)^{2}}{2 !}-\frac{(\Delta \Phi)^{4}}{4 !}+\ldots\right)\right)\right] \\
&+\frac{(\beta L)^{4}}{4 !}\left[-\frac{(\Delta \Phi)^{4}}{4 !}+\frac{(\Delta \Phi)^{6}}{6 !}-\ldots+\Delta \Phi\left(\frac{(\Delta \Phi)^{3}}{3 !}-\frac{(\Delta \Phi)^{5}}{5 !}+\ldots\right)\right. \\
&+\frac{(\Delta \Phi)^{2}}{2 !}\left(-\frac{(\Delta \Phi)^{2}}{2 !}+\frac{(\Delta \Phi)^{4}}{4 !}-\ldots\right)+\frac{(\Delta \Phi)^{3}}{3 !}\left(\Delta \Phi-\frac{(\Delta \Phi)^{3}}{3 !}+\ldots\right) \\
&++i\left(-\frac{(\Delta \Phi)^{5}}{5 !}+\frac{(\Delta \Phi)^{7}}{7 !}-\ldots+\Delta \Phi\left(\frac{\Delta \Phi)^{4}}{4 !}-\frac{(\Delta \Phi)^{6}}{6 !}+\ldots\right)\right. \\
&\left.\left.+\frac{(\Delta \Phi)^{2}}{2 !}\left(-\frac{(\Delta \Phi)^{3}}{3 !}+\frac{(\Delta \Phi)^{5}}{5 !}-\ldots\right)+\frac{(\Delta \Phi)^{3}}{3 !}\left(\frac{(\Delta \Phi)^{2}}{2 !}-\frac{(\Delta \Phi)^{4}}{4 !}+\ldots\right)\right)\right]+\ldots \\
&
\end{aligned}
$$

Noting that all the infinite series in $\Delta \Phi$ in (D2) are sine or cosine series, possibly with sequences of missing low order terms, enables the right side to be written as in the large curly brackets of (5.35). 
Factoring out the terms proportional to $S \equiv \sin \Delta \Phi$ and $C \equiv \cos \Delta \Phi$ at each order in $\beta L$, D2 may then be re-written as:

$$
\begin{aligned}
F_{\text {ref }} & =1+\beta L[S+i(1-C)] \\
& +\frac{(\beta L)^{2}}{2 !}[C-1+S \Delta \Phi+i(S-C \Delta \Phi)] \\
& +\frac{(\beta L)^{3}}{3 !}\left[-S\left(1-\frac{(\Delta \Phi)^{2}}{2 !}\right)+C \Delta \Phi+i\left(C\left(1-\frac{(\Delta \Phi)^{2}}{2 !}\right)+S \Delta \Phi-1\right)\right] \\
& +\frac{(\beta L)^{4}}{4 !}\left[1-C\left(1-\frac{(\Delta \Phi)^{2}}{2 !}\right)-S\left(\Delta \Phi-\frac{(\Delta \Phi)^{3}}{3 !}\right)\right. \\
& \left.+i\left(C\left(\Delta \Phi-\frac{(\Delta \Phi)^{3}}{3 !}\right)-S\left(1-\frac{(\Delta \Phi)^{2}}{2 !}\right)\right)\right] \\
& +\frac{(\beta L)^{5}}{5 !}\left[-C\left(\Delta \Phi-\frac{(\Delta \Phi)^{3}}{3 !}\right)+S\left(1-\frac{(\Delta \Phi)^{2}}{2 !}+\frac{(\Delta \Phi)^{4}}{4 !}\right)\right. \\
& \left.+i\left(1-S\left(\Delta \Phi-\frac{(\Delta \Phi)^{3}}{3 !}\right)-C\left(1-\frac{(\Delta \Phi)^{2}}{2 !}+\frac{(\Delta \Phi)^{4}}{4 !}\right)\right)\right] \\
& +\ldots
\end{aligned}
$$

The polynomials in $\Delta \Phi$ that multiply $S$ and $C$ at each order in $\beta L$ are the truncated sine and cosine series $S_{j}$ and $C_{j}$ respectively, defined in (5.36) and (5.37). Substitution of these series in (D3) yields then (5.38) and (5.39) of the text. 


\section{Appendix E}

It is assumed in the discussion of the Michelson interferometer in Section 8 that the source atom is at rest. In the case that the source is in motion in the laboratory frame the time factors in the complex exponentials of (8.1) and (8.2) must be scaled by the factor $1 / \gamma$ where $\gamma \equiv 1 / \sqrt{1-(v / c)^{2}}$ and $v$ is the source atom velocity, to take into account relativistic time dilatation. This is because the correct argument of the spacetime propagator (see (3.11)) is the proper time $\tau$, and $\Delta \tau=\Delta t / \gamma$ where $t$ is the laboratory time.

Assuming the Maxwellian distribution:

$$
\frac{d N}{d p}=C p^{2} e^{-\frac{p^{2}}{\bar{p}^{2}}}
$$

for the momentum, $p$, of the source atom, of mass, $M$, at absolute temperature, $T$, where $\bar{p}^{2}=2 M k T$, enables the distribution of the relativistic parameter $\gamma$ to be calculated via the relation:

$$
\gamma=\frac{E}{M}=\frac{\sqrt{M^{2}+p^{2}}}{M}=1+\frac{1}{2} \frac{p^{2}}{M^{2}}+\ldots
$$

Modifying the argument of the cosine in (8.7) to take into account the motion of the source atom gives

$$
\cos (2 \kappa d) \rightarrow \cos \left(\frac{2 \kappa d}{\gamma}\right) \simeq \cos \left(2 \kappa d-\frac{\kappa p^{2} d}{M^{2}}\right)
$$

Performing the average over $p$ using the distribution (E1):

$$
\left\langle\cos \left(\frac{2 \kappa d}{\gamma}\right)\right\rangle=\operatorname{Re} e^{2 i \kappa d}\left\{\frac{\int_{0}^{\infty} e^{-\frac{p^{2}}{\bar{p}^{2}}} e^{-\frac{i \kappa p^{2} d}{M^{2}}} d p}{\int_{0}^{\infty} e^{-\frac{p^{2}}{\bar{p}^{2}}} d p}\right\}
$$

Performing the integrals (see [75]) gives the result:

$$
\left\langle\cos \left(\frac{2 \kappa d}{\gamma}\right)\right\rangle=\operatorname{Re} \frac{k^{-\frac{3}{2}} e^{2 i \kappa d}}{\bar{p}^{3}}
$$

where

$$
k=\frac{1}{\bar{p}^{2}}\left[1+i \kappa\left(\frac{\bar{p}}{M}\right)^{2} d\right]
$$

Since $\kappa\left(\frac{\bar{p}}{M}\right)^{2} d \ll 1$, it follows that:

$$
k^{-\frac{3}{2}} \simeq \bar{p}^{3}\left[1-\frac{3}{2} i \kappa\left(\frac{\bar{p}}{M}\right)^{2} d\right] \simeq \bar{p}^{3} \exp \left[-\frac{3}{2} i \kappa\left(\frac{\bar{p}}{M}\right)^{2} d\right]
$$

Combining (E5) and (E6) gives finally:

$$
\left\langle\cos \left(\frac{2 \kappa d}{\gamma}\right)\right\rangle=\cos \left[2 \kappa d\left(1-\frac{3}{4}\left(\frac{\bar{p}}{M}\right)^{2}\right)\right]
$$

Thus the only effect of random Maxwellian motion of the source atom is the small relative change $-(3 / 4)(\bar{p} / M)^{2}$ of the phase of the interference term. There is no damping of the fringe visibility due to Doppler smearing of the wavelength of light as predicted by the classical wave theory. The numerical size of the correction term $3 / 4(\bar{p} / M)^{2}$ for a sodium atom at NTP is $1.6 \times 10^{-12}$. 


\section{Appendix F}

For convenience, units with $\hbar=c=1$ are employed in this Appendix. The following variables are used to characterise the velocities $v_{A}$ and $v_{B}$ of the electron in path $\mathrm{A}$ or path B:

$$
\alpha \equiv \frac{t_{A}}{t_{B}}, \quad \beta \equiv t_{A}-t_{B}
$$

The velocities are given, in terms of these variables and the path lengths $r^{\prime}+r_{A}$ and $r^{\prime}+r_{B}$ by the relations:

$$
v_{A}=\frac{(1-\alpha)\left(r^{\prime}+r_{A}\right)}{\beta}, \quad v_{B}=\frac{(1-\alpha)\left(r^{\prime}+r_{B}\right)}{\alpha \beta}
$$

The physical ranges of $\alpha$ and $\beta$ are:

$$
\begin{aligned}
& 1<\alpha<\frac{\left(r^{\prime}+r_{B}\right)}{\left(r^{\prime}+r_{A}\right)} \equiv \alpha_{\max } \\
& 0<\beta<\infty
\end{aligned}
$$

The limit $\beta \rightarrow \infty$ corresponds to vanishingly small velocity. The values $\alpha=1, \beta=0$ (in which case the ratio $(1-\alpha) / \beta$ remains finite) correspond to equal production times for any allowed velocity, whereas $\alpha=\alpha_{\max }$, for any $\beta$, corresponds to equal velocities. The equations (F2) give for the velocity difference:

$$
v_{B}-v_{A}=\frac{r^{\prime}(1-\alpha)}{\beta}\left[\left(\frac{1}{\alpha}-1\right)+\frac{1}{r^{\prime}}\left(\frac{r_{B}}{\alpha}-r_{A}\right)\right]
$$

and for the average velocity:

$$
\bar{v}=\frac{v_{B}+v_{A}}{2}=\frac{r^{\prime}(1-\alpha)}{2 \beta}\left[\left(\frac{1}{\alpha}+1\right)+\frac{1}{r^{\prime}}\left(\frac{r_{B}}{\alpha}+r_{A}\right)\right]
$$

Combining (F5) and (F6):

$$
\begin{aligned}
v_{B}-v_{A} & =2 \bar{v} \frac{\left(\frac{1}{\alpha}-1\right)+\frac{1}{r^{\prime}}\left(\frac{r_{B}}{\alpha}-r_{A}\right)}{\left(\frac{1}{\alpha}+1\right)+\frac{1}{r^{\prime}}\left(\frac{r_{B}}{\alpha}+r_{A}\right)} \\
& =\bar{v} \frac{\left(r^{\prime}+r_{A}\right)\left(\frac{\alpha_{\max }-1}{\alpha}\right)}{\left(r^{\prime}+\bar{r}\right)}+O\left[(\Delta r)^{2}\right] \\
& \equiv 2 \delta_{v}+O\left[(\Delta r)^{2}\right]
\end{aligned}
$$

To first order in $\Delta r$ :

$$
v_{A}=\bar{v}-\delta_{v} \quad, \quad v_{B}=\bar{v}+\delta_{v}
$$

From the relativistic relation:

$$
v=\frac{p}{E}=\frac{p}{\sqrt{\left(m^{2}+p^{2}\right)}}
$$

it follows that $\delta_{v}=\left(m^{2} / E^{3}\right) \delta_{p}$ so that

$$
p_{A}=\bar{p}-\delta_{p} \quad, \quad p_{B}=\bar{p}+\delta_{p}
$$


where

$$
\delta_{p}=\frac{\bar{E}^{3}}{m^{2}} \delta_{v}=\frac{\bar{p}\left(1+\left(\frac{\bar{p}}{m}\right)^{2}\right)\left(r^{\prime}+r_{A}\right)\left(\frac{\alpha_{\max }}{\alpha}-1\right)}{2\left(r^{\prime}+\bar{r}\right)}
$$

Substituting now (F9) into the formula (9.15) for the phase difference, retaining only first order terms in $\delta_{p}$, and using (F10), gives:

$$
\begin{aligned}
\phi_{B}-\phi_{A} & =m^{2}\left[-\frac{\Delta r}{\bar{p}}+2\left(r^{\prime}+\bar{r}\right) \frac{\delta_{p}}{\bar{p}^{2}}\right] \\
& =m^{2}\left[-\frac{\Delta r}{\bar{p}}+\left(\frac{\bar{p}}{m^{2}}+\frac{1}{\bar{p}}\right)\left(r^{\prime}+r_{A}\right)\left(\frac{\alpha_{\max }}{\alpha}-1\right)\right]
\end{aligned}
$$

Substituting $\alpha=\alpha_{\max }$ in (F11), corresponding to $v_{A}=v_{B}$, gives

$$
\phi_{B}-\phi_{A}=-\frac{m^{2} \Delta r}{\bar{p}} \quad \text { (equal velocities) }
$$

while setting $\alpha=1$ or $t_{A}=t_{B}$ gives:

$$
\phi_{B}-\phi_{A}=\bar{p} \Delta r \quad \text { (equal production times) }
$$

For non-relativistic particles for which $\bar{p} \ll m$ the equal velocity relation (F12) gives an effective de Broglie wavelength much shorter than (9.19) that is applicable in the equal production time case, (F13), and which is in good agreement with experiment [69]. .

\section{References}

[1] R.P.Feynman, 'QED, The Strange Theory of Light and Matter', Princeton University Press, New Jersey, 1985.

[2] 'Isaac Newton's Papers and Letters on Natural Philosophy', Harvard University Press, Cambridge, MA 1958. See also I.B.Cohen and R.S.Westfall, 'Newton' W.W. Norton Company, New York, 1995, pp 181-184.

[3] R.P.Feynman, R.B.Leighton and M.Sands, 'The Feynman Lectures in Physics' Addison-Wesley, Reading Massachusetts, 1963, Vol 1 Section 33-6.

[4] Reference [3], Section 26-6.

[5] Reference [3], Vol 1 Chapter 37, Vol 2 Section 15-3, Vol 3 Chapter 3.

[6] R.P.Feynman. Rev. Mod. Phys. 20367 (1948).

[7] R.P.Feynman and A.R.Hibbs, 'Quantum Mechanics and Path Integrals', McGraw Hill, New York, 1965.

[8] E.F.Taylor, Am. J. Phys. 66369 (1998); 71423 (2003).

[9] N.Bohr, Nature 121580 (1928).

[10] J.M.Lévy-Leblond and F.Balibar 'Quantics', North Holland, Amsterdam, 1990. 
[11] J.H.Field, Eur. Phys. J. C30 305 (2003).

[12] J.H.Field, Eur. Phys. J. C37 359 (2004).

[13] J.H.Field, 'Lepton Flavour Eigenstates do not Exist if Neutrinos are Massive: "Neutrino Oscillations" Reconsidered',arXiv hep-ph/0301231.

[14] B.G. de Grooth, Am. J. Phys. 651165 (1997).

[15] A.O.Barut and S.Basri, Am. J. Phys. 60 896(1992).

[16] C.Blondel, S.Berge and C.Debart, Am. J. Phys. 69 810(2001), and references therein.

[17] W.Heisenberg, 'The Physical Principals of the Quantum Theory', English Translation by C.Eckart and F.C.Hoyt, University of Chicago Press, Chicago, 1930. Chapter IV, Section 2.

[18] Reference [6], Equation (38).

[19] P.A.M.Dirac, Physikalische Zeitschrift der Sowjetunion Band 3, Heft 1 (1933). Reprinted in 'Selected Papers on Quantum Electrodynamics', Ed. J.Schwinger, Dover, New York, (1958) P312;

'The Principles of Quantum Mechanics', Fourth Edition, O.U.P., London (1958), Chapter V, Section 32.

[20] Second Reference in [19], Section 30, Eqn(29).

[21] W.Heisenberg, Z. Für Physik, 43172 (1927). English translation in 'Quantum Theory of Measurement' Eds, A.Wheeler and W.H.Zurek, Princeton University Press, 1983, P62.

[22] R.P.Feynman, 'The Character of Physical Law', The M.I.T. Press, Cambridge MA, 1967, P130, and Reference [3] Vol III, Chapter 1.

[23] R.Griffiths, J. Stat. Phys. 36219 (1984).

[24] R.Omnès, Rev. Mod. Phys. 64339 (1992): 'The Interpretation of Quantum Mechanics', Princeton University Press, 1994.

[25] M.Gell-Mann and J.B.Hartle, Phys. Rev. D47 3345 (1993).

[26] L. de Broglie, 'Nouvelles Perspectives en Microphysique', Albin Michel, Paris, 1956.

[27] L. de Broglie, in 'Electrons and Photons, The Proceedings of the Fifth Solvay Conference 1927', Translated and edited by G.Bacciagaluppa and A.Valentin, C.U.P. to be published.

[28] D.Bohm and B.J.Hiley, Phys. Rep. 144321 (1987); 'The Undivided Universe', Routledge, London, 1993.

[29] D.S.Sivia, 'Data Analysis -A Bayesian Tutorial', O.U.P., Oxford, 1997.

[30] A.J.Leggett, Supl. of Prog. Theor. Phys. No 6980 (1980).

[31] Reference [7], Section 4.1, Eqn 43. 
[32] H.Goldstein, 'Classical Mechanics', Addison-Wesley MA, 1959, Section 6-5, Eqn 6-49.

[33] M.Born and E.Wolf, 'Principles of Optics', Pergamon Press, Oxford, 1964, Section 8.2 .

[34] R.P.Feynman, Phys. Rev. 76749 (1949).

[35] Second Reference in [19], Section 28, Eqn 20.

[36] Reference [33], Section 8.3.

[37] L.Mandel and E.Wolf, 'Optical Coherence and Quantum Optics' C.U.P., Cambridge, 1995, Section 3.2.

[38] Reference [37] Section 4.2.1 P150.

[39] See the discussion of Fig 1-10 in Reference [7]

[40] J.J.Sakurai, 'Advanced Quantum Mechanics', Addison-Wesley, Redwood City CA, 1967, $\operatorname{Eqn}(2.215)$ P62.

[41] Reference [33], Section 1.5, Eqns(23).

[42] F.A.Jenkins and H.E.White, 'Fundamentals of Optics' Mc Graw-Hill, New York, 1959, Ch 13.

[43] Reference [37] Section 7.3, Fig 7.4.

[44] A.A.Michelson, 'Studies in Optics' University of Chicago Press, Chicago, 1927, Chapter IV.

[45] J.Cooper, Reports on Progress in Physics, Vol. XXIX, 35 (1996) and references therein.

[46] R.G.Breene, Rev. Mod. Phys. 3994 (1957).

[47] E.U.Condon and G.H.Shortley, 'The Theory of Atomic Spectra' C.U.P., Cambridge, 1967.

[48] R.W.Ditchburn, 'Light', Blackie and Son, London. 1959, Ch18 p601.

[49] A.Tonomura et. al Am. J. Phys. 57117 (1989).

[50] Y.Tsuchiya et. al in 'Advances in Electronics and Electron Physics' edited by P.Hawkes (Academic Press, New York. 1982, Vol 64A, P21).

[51] J.C.Hart et. al Nucl. Phys.B 66317 (1973).

[52] R.Kleiss in 'Proceedings of the 1989 CERN-JINR School of Physics', CERN Yellow Report 91-07, P103.

[53] S. De Leo and P.Rotelli, JETP Lett. 7656 (2002).

[54] G.S.Gjesdal et. al, Phys. Lett. B 52113 (1974). 
[55] A.Angelopoulos et. al Phys. Lett. B 44438 (1998).

[56] S.Eidelman et. al 'Review of Particle Properties', Phys. Lett. B 592 (2004).

[57] Z.Maki, M.Nakagawa and S.Sakata, Prog. Theor. Phys. 28870 (1962).

[58] B.Kayser in Reference [56], P145.

[59] S.De Leo, G.Ducati and P.Rotelli, Mod. Phys. Lett. A 152057 (2000).

[60] L.B.Okun and I.S.Tsukerman, Mod. Phys. Lett. A 151481 (2000).

[61] C.Giunti, Physica Scripta, 6729 (2003); 'Coherence in Neutrino Oscillations' hep$\mathrm{ph} / 0302045$.

[62] J.H.Field, arXiv pre-prints hep-ph/0303241, $0206300,0401051$.

[63] M.H.Ahn et. al Phys.Rev.Lett.90 041801 (2003).

[64] Remark of E.J.Williams quoted in 'Our Universes', D.H.Wilkinson, Columbia University Press, 1991, P69.

[65] P.Spezial Ed 'Albert Einstein-Michele Besso Correspondence 1903-1955, Hermann, Paris 1972. Letter dated 12 December 1951 P453.

[66] J.H.Field, Helv. Phys. Acta. 70452 (1997),arXiv physics/0410262.

[67] J.H.Field, Eur. J. Phys. 25385 (2004), arXiv physics/0403077.

[68] J.H.Field, 'Classical Electrodynamics as a Consequence of Coulomb's Law, Special Relativity and Hamilton's Principle, and its Relationship to Quantum Electrodynamics', arXiv physics/0501130.

[69] C.Davisson and L.H.Germer, Phys. Rev. 30705 (1927).

[70] L.Wolfenstein, Phys. Rev.D 172369 (1976).

[71] S.P.Mikheev and A. Yu Smirnov, Sov. J. Nucl. Phys. 42913 (1985); Il Nuovo Cimento 9 C 17 (1986).

[72] A.Pais and O.Piccioni, Phys. Rev. 1001487 (1955).

[73] P.Roetling, 'The Physics of Digital Colour', Physics Today, December 1992, P23.

[74] Reference [42], Ch 17.

[75] J.Jeans, 'An Introduction to the Kinetic Theory of Gases', C.U.P. Cambridge 1959, Appendix VI. 